Acta Math., 218 (2017), 137-199

DOI: 10.4310/ACTA.2017.v218.n1.a3

(C) 2017 by Institut Mittag-Leffler. All rights reserved

\title{
Equivariant Dirac operators and differentiable geometric invariant theory
}

by

Paul-Emile Paradan

CNRS UMR 5149

Université de Montpellier

Montpellier, France

\author{
MichÈLe Vergne \\ CNRS UMR 7586 \\ Université Paris 7 \\ Paris, France
}

\section{Contents}

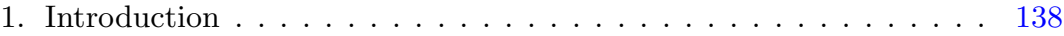

1.1. Description of the results . . . . . . . . . . . . . . 141

1.2. Strategy . . . . . . . . . . . . . . . . . . . . . 144

1.3. Outline of the article . . . . . . . . . . . . . . 146

Acknowledgments . . . . . . . . . . . . . . . . . 147

Notation . . . . . . . . . . . . . . . . . . . . 147

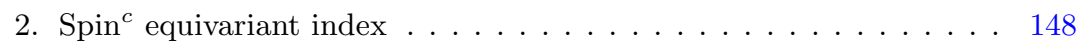

2.1. Spin ${ }^{c}$ modules . . . . . . . . . . . . . . . . . . . . . 148

2.2. Spin $^{c}$ structures . . . . . . . . . . . . . . . . 150

2.3. Moment maps and Kirwan vector field . . . . . . . . . . . . 152

2.4. Equivariant index . . . . . . . . . . . . . . . . 153

3. Coadjoint orbits . . . . . . . . . . . . . . . . 155

3.1. Admissible coadjoint orbits . . . . . . . . . . . . . . 155

3.2. Magical inequality . . . . . . . . . . . . . . . . . . . 159

3.3. Slices and induced $\operatorname{spin}^{c}$-bundles . . . . . . . . . . . . 160

4. Computing the multiplicities . . . . . . . . . . . . . . . 162

4.1. Transversally elliptic operators . . . . . . . . . . . . . . 162

4.2. The Witten deformation . . . . . . . . . . . . . . . 164

4.3. Some properties of the localized index . . . . . . . . . . . 166

4.4. The function $d_{\mathcal{S}} \ldots \ldots \ldots \ldots \ldots \ldots \ldots$

4.5. The Witten deformation on the product $M \times \mathcal{O}^{*} \ldots \ldots$. . . 172

5. Multiplicities and reduced spaces . . . . . . . . . . . . . 181

5.1. Spin ${ }^{c}$ index on singular reduced spaces . . . . . . . . . . . . . . . . . . . . . . . . 182

5.2. Proof of Theorem $5.4 \ldots \ldots \ldots \ldots$. . . . . . . . . . 184

5.3. $[Q, R]=0 \ldots \ldots \ldots \ldots \ldots \ldots \ldots \ldots \ldots \ldots$ 
6. Examples: multiplicities and reduced spaces . . . . . . . . . . . 188

6.1. The reduced space might not be connected . . . . . . . . . . . 189

6.2. The image of the moment map might be non-convex . . . . . . 191

6.3. The multiplicity of the trivial representation comes from two reduced spaces . . . . . . . . . . . . . . . 196

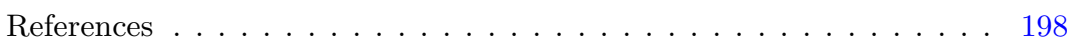

\section{Introduction}

When $D$ is an elliptic operator on a manifold $M$ preserved by a compact group $K$ of symmetry, one can understand the aim of "geometric invariant theory" as the realization of the space of $K$-invariant solutions of $D$ as the space of solutions of an elliptic operator on a "geometric quotient" $M_{0}$ of $M$.

The by now classical case is concerned with a $K$-action on a compact complex manifold $M$ : we may consider the Dolbeault operator $D$ acting on sections of a holomorphic line bundle $L$. When $L$ is ample Guillemin-Sternberg [13] proved that the $K$-invariant solutions of $D$ can be realized on Mumford's GIT quotient $M_{0}:=\Phi_{L}^{-1}(0) / K$ : here $\Phi_{L}$ is the moment map associated with the $K$-action on the line bundle $L$. This result was extended to other cohomology groups by Teleman in [32] (see also [31]).

In our article, we show that the same construction can be generalized to the differentiable case if properly reformulated. We consider a compact connected Lie group $K$ with Lie algebra $\mathfrak{k}$ acting on a compact, oriented and even-dimensional manifold $M$. In this introduction we assume for simplicity that $M$ carries a $K$-invariant spin structure: the corresponding Dirac operator plays the role of the Dolbeault operator.

For any line bundle $L$ we consider the Dirac operator $D:=D_{L}$ twisted by $L$. It acts on sections of the Clifford bundle $\mathcal{S}=\mathcal{S}_{\text {spin }} \otimes L$ on $M$, where $\mathcal{S}_{\text {spin }}$ is the spinor bundle of $M$. We are concerned with the equivariant index of $D$, that we denote by $\mathcal{Q}_{K}(M, \mathcal{S})$, and we also say that $\mathcal{Q}_{K}(M, \mathcal{S})$ is the space of virtual solutions of $D$. It belongs to the Grothendieck group of representations of $K$. More generally, we can consider any irreducible equivariant Clifford module $\mathcal{S}$ over $M$, when $M$ admits a $\operatorname{spin}^{c}$ structure.

An important example is when $M$ is a compact complex manifold, $K$ a compact group of holomorphic transformations of $M, L$ a holomorphic $K$-equivariant line bundle on $M$, not necessarily ample, and $D$ the Dolbeault operator acting on sections on the Clifford bundle $\mathcal{S}$ of $L$-valued differential forms of type $(0, q)$. Then

$$
\mathcal{Q}_{K}(M, \mathcal{S})=\sum_{q=0}^{\operatorname{dim}_{\mathbb{C}} M}(-1)^{q} H^{0, q}(M, L)
$$


Our aim is to show that the virtual space of $K$-invariant solutions of the twisted Dirac operator $D$ can be identified to the space of virtual solutions of a twisted Dirac operator on a "geometric quotient" $M_{0}$ of $M$, constructed with the help of a moment map. To formulate a clean result in the context of Dirac operators is not obvious. Let us first state the vanishing theorem (surprisingly difficult to prove) which will allow us to do so.

We use Duflo's notion of admissible coadjoint orbits (see §3) to produce unitary irreducible representations of $K$. There is a map $\mathrm{Q}_{K}^{\text {spin }}$ associating with an admissible coadjoint orbit $\mathcal{P}$ a virtual representation $\mathrm{Q}_{K}^{\text {spin }}(\mathcal{P})$ of $K$. By this correspondence, regular admissible coadjoint orbits parameterize the set $\widehat{K}$ of classes of unitary irreducible representations of $K$. The coadjoint orbit of $\varrho$ is regular admissible and parameterizes the trivial representation of $K$. However, if $r$ is the rank of $[\mathfrak{k}, \mathfrak{k}]$, there are $2^{r}$ admissible orbits $\mathcal{P}$ such that $\mathrm{Q}_{K}^{\mathrm{spin}}(\mathcal{P})$ is the trivial representation of $K$. We will say that such an orbit $\mathcal{P}$ is an ancestor of the trivial representation.

For $\mathfrak{h}$ a subalgebra of $\mathfrak{k}$, we denote by $(\mathfrak{h})$ the conjugacy class of $\mathfrak{h}$. If $\xi \in \mathfrak{k}^{*}$, we denote by $\mathfrak{k}_{\xi}$ its infinitesimal stabilizer. The set $\mathcal{H}_{\mathfrak{k}}$ of conjugacy classes of the algebras $\mathfrak{k}_{\xi}, \xi$ running in $\mathfrak{k}^{*}$, is a finite set. Indeed the complexified Lie algebras of $\mathfrak{k}_{\xi}$ varies over the Levi subalgebras of $\mathfrak{k}_{\mathbb{C}}$. For $(\mathfrak{h}) \in \mathcal{H}$, we say that a coadjoint orbit $K \xi$ is of type (h) if $\mathfrak{k}_{\xi}$ belongs to the conjugacy class $(\mathfrak{h})$. The semi-simple part of $\mathfrak{k}_{\xi}$ is $\left[\mathfrak{k}_{\xi}, \mathfrak{k}_{\xi}\right]$.

Let $\left(\mathfrak{k}_{M}\right)$ be the generic infinitesimal stabilizer of the $K$-action on $M$. We prove the following result.

THEOREM 1.1. If $\left(\left[\mathfrak{k}_{M}, \mathfrak{k}_{M}\right]\right)$ is not equal to some $([\mathfrak{h}, \mathfrak{h}])$, for $\mathfrak{h} \in \mathcal{H}_{\mathfrak{k}}$, then for any $K$-equivariant line bundle $\mathcal{L}, \mathcal{Q}_{K}(M, \mathcal{S})=0$.

We may thus assume that there exists $(\mathfrak{h}) \in \mathcal{H}_{\mathfrak{k}}$ such that $\left(\left[\mathfrak{k}_{M}, \mathfrak{k}_{M}\right]\right)=([\mathfrak{h}, \mathfrak{h}])$ : this class is unique and is denoted by $\left(\mathfrak{h}_{M}\right)$. This condition on the $K$-action is always satisfied in the Hamiltonian setting [21], but not always in the spin setting (see the case of spheres in Example 4.23).

Consider our line bundle $L$. The choice of a Hermitian connection $\nabla$ determines a moment map

$$
\Phi_{L}: M \longrightarrow \mathfrak{k}^{*}
$$

by the relation $\mathcal{L}(X)-\nabla_{X_{M}}=i\left\langle\Phi_{L}, X\right\rangle$, for all $X \in \mathfrak{k}$.

We now describe the geometric quotient $M_{0}$. Let us first state the result, when the infinitesimal stabilizer $\left(\mathfrak{k}_{M}\right)$ is abelian. The corresponding $\left(\mathfrak{h}_{M}\right)$ is the conjugacy class of Cartan subalgebras, and we consider

$$
M_{0}=\Phi_{L}^{-1}(K \varrho) / K
$$


where $K \varrho$ is the regular admissible orbit that parametrizes the trivial representation. In the general case, we define $\mathcal{O}_{M}=\bigcup \mathcal{P}$ to be the union of the ancestors of the trivial representation which are of type $\left(\mathfrak{h}_{M}\right)$. Thus $\mathcal{O}_{M}$ is a union of a finite number of admissible coadjoint orbits, a number that might be greater than 1 (see the example in $\S 6.3$ ). We then consider

$$
M_{0}=\Phi_{L}^{-1}\left(\mathcal{O}_{M}\right) / K
$$

Then, we define by a desingularization procedure, a virtual vector space $\mathrm{Q}^{\operatorname{spin}}\left(M_{0}\right)$, which coincides when $M_{0}$ is smooth to the space of virtual solutions of a twisted Dirac operator on $M_{0}$. We prove the following theorem.

TheOrem 1.2.

$$
\left[\mathcal{Q}_{K}(M, \mathcal{S})\right]^{K}=\mathrm{Q}^{\mathrm{spin}}\left(M_{0}\right)
$$

This is an equality of dimensions. This equality also holds in the Grothendieck group of irreducible representations of $G$, if $G$ is a compact group of symmetry commuting with the action of $K$.

Thus our space $M_{0}$ plays the role of the geometric quotient in this purely differentiable setting. The space $M_{0}$ may vary dramatically with the choice of the connextion $\nabla$, but not its quantized space $\mathrm{Q}^{\operatorname{spin}}\left(M_{0}\right)$.

Let us recall that we did not make any assumption on the line bundle $L$. So this equality is true for any line bundle $L$, and any choice of $K$-invariant connection $\nabla$ on $L$. In particular, the curvature of $\nabla$ might be always degenerate, whatever choice of connection. In $\S 6$, we raise a question on existence of "best connections".

Let us recall the previous results on this subject. After their work [13] GuilleminSternberg formulated the conjecture "Quantization commutes with reduction" denoted by $[Q, R]=0$. This conjecture was proved in full generality by Meinrenken-Sjamaar [24], following partial results notably by [12], [34], [35], [18], [23]. Later, other proofs by analytic or topological methods were given by [33], [26].

After the remarkable results of Meinrenken-Sjamaar [24], it was tempting to find in what way we can extend their results to the general $\operatorname{spin}^{c}$ situation. In this general context, our manifold $M$ is not necessarily complex, nor even almost-complex. So the only elliptic operators which make sense in this case are twisted Dirac operators. We restrict ourselves to line bundles, the case of vector bundles being obtained by pushforward of index of line bundles.

When $M$ is a $\operatorname{spin}^{c}$ manifold, with an action of $S^{1}$, a partial answer to the question of quantization commutes with reduction in the spin setting has been obtained by [10], [11], [30]. The case of toric manifolds and non ample line bundles has been treated in [19]. These interesting examples (we give an example due to Karshon-Tolman in 
§6) motivated us to search for a general result. However, to formulate what should be the result in the general non-abelian case was not immediately clear to us, although a posteriori very natural. We really had to use (in the case where the generic stabilizer is non-abelian) non regular admissible orbits.

Let us also say that, due to the inevitable $\varrho$-shift in the spin context, our theorem does not imply immediately the $[Q, R]=0$ theorem of the Hamiltonian case. Both theorems are somewhat magical, but each one on its own. We will come back to the comparison between these two formulations in future work devoted to the special case of almost complex manifolds.

Recently, using analytic methods adapted from those of Braverman, Ma, Tian and Zhang [33], [6], [22], [7], Hochs-Mathai [16] and Hochs-Song [17] have extended our theorem to other natural settings where the group and/or the manifold are not compact. Note that in their works, the authors have to use our result in the compact setting to obtain these extensions.

\subsection{Description of the results}

We now give a detailed description of the theorem proved in this article.

Let $M$ be a compact connected manifold. We assume that $M$ is even-dimensional and oriented. We consider a $\operatorname{spin}^{c}$ structure on $M$, and denote by $\mathcal{S}$ the corresponding spinor bundle. Let $K$ be a compact connected Lie group acting on $M$ and $\mathcal{S}$, and we denote by $D: \Gamma\left(M, \mathcal{S}^{+}\right) \rightarrow \Gamma\left(M, \mathcal{S}^{-}\right)$the corresponding $K$-equivariant $\operatorname{spin}^{c}$ Dirac operator.

Our aim is to describe the space of $K$-invariant solutions, or more generally, the equivariant index of $D$, denoted by $\mathcal{Q}_{K}(M, \mathcal{S})$. It belongs to the Grothendieck group of representations of $K$ :

$$
\mathcal{Q}_{K}(M, \mathcal{S})=\sum_{\pi \in \widehat{K}} \mathrm{~m}(\pi) \pi .
$$

Consider the determinant line bundle $\operatorname{det}(\mathcal{S})$ of the $\operatorname{spin}^{c}$ structure. This is a $K$ equivariant complex line bundle on $M$. The choice of a $K$-invariant Hermitian metric and of a $K$-invariant Hermitian connection $\nabla$ on $\operatorname{det}(\mathcal{S})$ determines a moment map

$$
\Phi_{\mathcal{S}}: M \longrightarrow \mathfrak{k}^{*} .
$$

If $M$ is spin and $\mathcal{S}=\mathcal{S}_{\text {spin }} \otimes L$, then $\operatorname{det}(\mathcal{S})=L^{\otimes 2}$ and $\Phi_{\mathcal{S}}$ is equal to the moment map $\Phi_{L}$ associated with a connection on $L$.

We start to explain our result on the geometric description of $\mathrm{m}(\pi)$ in the torus case. The general case reduces (in spirit) to this case, using an appropriate slice for the $K$-action on $M$. 
Let $K=T$ be a torus acting effectively on $M$. In contrast to the symplectic case, the image $\Phi_{\mathcal{S}}(M)$ might not be convex. Let $\Lambda \subset \mathfrak{t}^{*}$ be the lattice of weights. If $\mu \in \Lambda$, we denote by $\mathbb{C}_{\mu}$ the corresponding 1-dimensional representation of $T$. The equivariant index $\mathcal{Q}_{T}(M, \mathcal{S})$ decomposes as $\mathcal{Q}_{T}(M, \mathcal{S})=\sum_{\mu \in \Lambda} \mathrm{m}_{\mu} \mathbb{C}_{\mu}$.

The topological space $M_{\mu}=\Phi_{\mathcal{S}}^{-1}(\mu) / T$, which may not be connected, is an orbifold provided with a $\operatorname{spin}^{c}$-structure when $\mu$ in $\mathfrak{t}^{*}$ is a regular value of $\Phi_{\mathcal{S}}$. In this case we define the integer $\mathrm{Q}^{\mathrm{spin}}\left(M_{\mu}\right)$ as the index of the corresponding $\operatorname{spin}^{c}$ Dirac operator on the orbifold $M_{\mu}$. We can define $\mathrm{Q}^{\text {spin }}\left(M_{\mu}\right)$ even if $\mu$ is a singular value. Postponing this definition, our result states that

$$
\mathrm{m}_{\mu}=\mathrm{Q}^{\mathrm{spin}}\left(M_{\mu}\right), \quad \text { for all } \mu \in \Lambda \text {. }
$$

Here is the definition of $\mathrm{Q}^{\operatorname{spin}}\left(M_{\mu}\right)$ (see $\S 5.1$ ). We approach $\mu$ by a regular value $\mu+\varepsilon$, and we define $\mathrm{Q}^{\mathrm{spin}}\left(M_{\mu}\right)$ as the index of a $\operatorname{spin}^{c}$ Dirac operator on the orbifold $M_{\mu+\varepsilon}$, and this is independent of the choice of $\varepsilon$ sufficiently close. Remark here that $\mu$ has to be an interior point of $\Phi_{\mathcal{S}}(M)$ in order for $\mathrm{Q}^{\text {spin }}\left(M_{\mu}\right)$ to be non zero, as otherwise we can take $\mu+\varepsilon$ not in the image. In a forthcoming article, we will give a more detailed description of the function $\mu \rightarrow \mathrm{Q}^{\text {spin }}\left(M_{\mu}\right)$ in terms of locally quasi-polynomial functions on $\mathfrak{t}^{*}$.

The identity (1.1) was obtained by Karshon-Tolman [19] when $M$ is a toric manifold, by Grossberg-Karshon [11] when $M$ is a locally toric space, and by Cannas da SilvaKarshon-Tolman [30] when $\operatorname{dim} T=1$. In Figure 1, we draw the picture of the function $\mu \mapsto \mathrm{Q}^{\text {spin }}\left(M_{\mu}\right)$ for the Hirzebruch surface, and a non ample line bundle on it (we give the details of this example due to Karshon-Tolman in the last section). The image of $\Phi$ is the union of the two large triangles in red and blue. The multiplicities are 1 on the integral points of the interior of the red triangle, and -1 on the integral points of the interior of the blue triangle.

Now consider the general case of a compact connected Lie group $K$ acting on $M$ and $\mathcal{S}$. So we may assume that $\left(\left[\mathfrak{k}_{M}, \mathfrak{k}_{M}\right]\right)=\left(\left[\mathfrak{h}_{M}, \mathfrak{h}_{M}\right]\right)$ for $\left(\mathfrak{h}_{M}\right) \in \mathcal{H}_{\mathfrak{k}}$, as otherwise $\mathcal{Q}_{K}(M, \mathcal{S})=0$.

We say that a coadjoint orbit $\mathcal{P} \subset \mathfrak{k}^{*}$ is admissible if $\mathcal{P}$ carries a spin ${ }^{c}$-bundle $\mathcal{S}_{\mathcal{P}}$ such that the corresponding moment map is the inclusion $\mathcal{P} \hookrightarrow \mathfrak{k}^{*}$. We denote simply by $\mathrm{Q}_{K}^{\text {spin }}(\mathcal{P})$ the element $\mathcal{Q}_{K}\left(\mathcal{P}, \mathcal{S}_{\mathcal{P}}\right) \in R(K)$. It is either zero or an irreducible representation of $K$, and the map

$$
\mathcal{O} \longmapsto \pi_{\mathcal{O}}:=\mathrm{Q}_{K}^{\text {spin }}(\mathcal{O})
$$

defines a bijection between the regular admissible orbits and the dual $\widehat{K}$. When $\mathcal{O}$ is a regular admissible orbit, an admissible coadjoint orbit $\mathcal{P}$ is called an ancestor of $\mathcal{O}$ (or a $K$-ancestor of $\left.\pi_{\mathcal{O}}\right)$ if $\mathrm{Q}_{K}^{\text {spin }}(\mathcal{P})=\pi_{\mathcal{O}}$. 


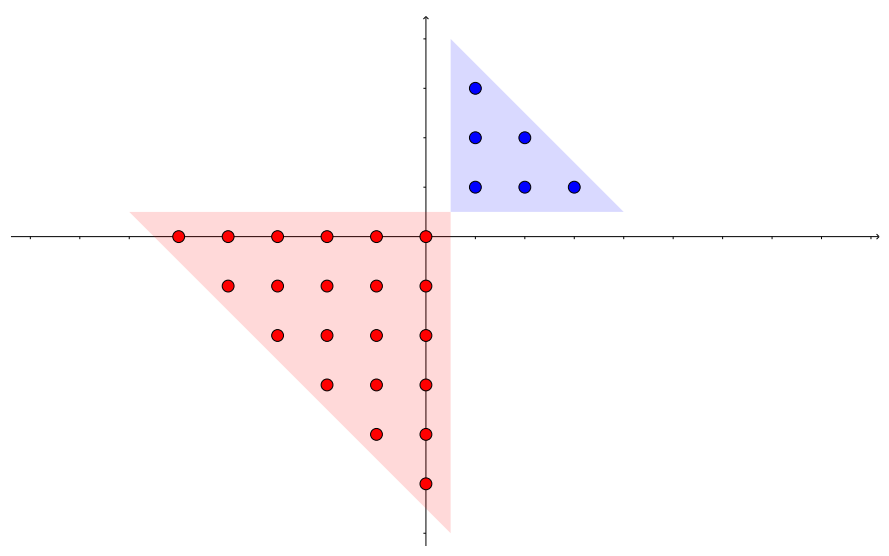

Figure 1. T-multiplicities for non ample bundle on Hirzebruch surface.

Denote by $\mathcal{A}\left(\left(\mathfrak{h}_{M}\right)\right)$ the set of admissible orbits of type $\left(\mathfrak{h}_{M}\right)$. If $\mathcal{P} \in \mathcal{A}\left(\left(\mathfrak{h}_{M}\right)\right)$, we can define the $\operatorname{spin}^{c}$ index $\mathrm{Q}^{\operatorname{spin}}\left(M_{\mathcal{P}}\right) \in \mathbb{Z}$ of the reduced space $M_{\mathcal{P}}=\Phi_{\mathcal{S}}^{-1}(\mathcal{P}) / K$ (by a deformation procedure if $M_{\mathcal{P}}$ is not smooth).

We obtain the following theorem which is the main result of the paper.

THEOREM 1.3. Assume that $\left(\left[\mathfrak{k}_{M}, \mathfrak{k}_{M}\right]\right)=\left(\left[\mathfrak{h}_{M}, \mathfrak{h}_{M}\right]\right)$ for $\left(\mathfrak{h}_{M}\right) \in \mathcal{H}_{\mathfrak{k}}$.

The multiplicity of the representation $\pi_{\mathcal{O}}$ in $\mathcal{Q}_{K}(M, \mathcal{S})$ is equal to

$$
\sum_{\mathcal{P}} \mathrm{Q}^{\operatorname{spin}}\left(M_{\mathcal{P}}\right)
$$

where the sum runs over the ancestors of $\mathcal{O}$ of type $\left(\mathfrak{h}_{M}\right)$. In other words,

$$
\mathcal{Q}_{K}(M, \mathcal{S})=\sum_{\mathcal{P} \in \mathcal{A}\left(\left(\mathfrak{h}_{M}\right)\right)} \mathrm{Q}^{\text {spin }}\left(M_{\mathcal{P}}\right) \mathrm{Q}_{K}^{\text {spin }}(\mathcal{P})
$$

When we consider the orbit $K \varrho$, the multiplicity of the representation $\pi_{K \varrho}$ in $\mathcal{Q}_{K}(M, \mathcal{S})$ is the space of $K$-invariant virtual solutions of $D$ and Theorem 1.3 implies Theorem 1.2.

It may be useful to rephrase this theorem by describing the parametrization of admissible orbits by parameters belonging to the closed Weyl chamber $\mathfrak{t}_{\geqslant 0}^{*}$. Let

$$
\Lambda_{\geqslant 0}:=\Lambda \cap \mathfrak{t}_{\geqslant 0}^{*}
$$

be the set of dominant weights, and let $\varrho$ be the half-sum of the positive roots. 
The set of regular admissible orbits is indexed by the set $\Lambda_{\geqslant 0}+\varrho$ : if $\lambda \in \Lambda_{\geqslant 0}+\varrho$, the coadjoint orbit $K \lambda$ is regular admissible and $\pi_{K \lambda}$ is the representation with highest weight $\lambda-\varrho$.

Denote by $\mathcal{F}$ the set of the relative interiors of the faces of $\mathfrak{t}_{\geqslant 0}^{*}$. Thus $\mathfrak{t}_{\geqslant 0}^{*}=\coprod_{\sigma \in \mathcal{F}} \sigma$. The face $\mathfrak{t}_{>0}^{*}$ is the open face in $\mathcal{F}$.

Let $\sigma \in \mathcal{F}$. The stabilizer $K_{\xi}$ of a point $\xi \in \sigma$ depends only of $\sigma$. We denote it by $K_{\sigma}$, and by $\mathfrak{k}_{\sigma}$ its Lie algebra. We choose on $\mathfrak{k}_{\sigma}$ the system of positive roots compatible with $\mathfrak{t}_{\geqslant 0}^{*}$, and let $\varrho^{K_{\sigma}}$ be the corresponding $\varrho$. When $\mu \in \sigma$, the coadjoint orbit $K \mu$ is admissible if and only if $\lambda=\mu-\varrho+\varrho^{K_{\sigma}} \in \Lambda$.

The map $\mathcal{F} \rightarrow \mathcal{H}_{\mathfrak{k}}, \sigma \mapsto\left(\mathfrak{k}_{\sigma}\right)$, is surjective but not injective. We denote by $\mathcal{F}(M)$ the set of faces of $\mathfrak{t}_{\geqslant 0}^{*}$ such that $\left(\mathfrak{k}_{\sigma}\right)=\left(\mathfrak{h}_{M}\right)$.

Using the above parameters, we may rephrase Theorem 1.3 as follows.

Theorem 1.4. Assume that $\left(\left[\mathfrak{k}_{M}, \mathfrak{k}_{M}\right]\right)=\left(\left[\mathfrak{h}_{M}, \mathfrak{h}_{M}\right]\right)$ with $\left(\mathfrak{h}_{M}\right) \in \mathcal{H}_{\mathfrak{k}}$. Let $\lambda \in \Lambda_{\geqslant 0}+\varrho$ and let $\mathrm{m}_{\lambda} \in \mathbb{Z}$ be the multiplicity of the representation $\pi_{K \lambda}$ in $\mathcal{Q}_{K}(M, \mathcal{S})$. We have

$$
\mathrm{m}_{\lambda}=\sum_{\substack{\sigma \in \mathcal{F}(M) \\ \lambda-\varrho^{K_{\sigma} \in \sigma}}} \mathrm{Q}^{\operatorname{spin}}\left(M_{K\left(\lambda-\varrho^{K_{\sigma}}\right)}\right) .
$$

More explicitly, the sum (1.2) is taken over the faces $\sigma$ of the Weyl chamber such that

$$
\left(\left[\mathfrak{k}_{M}, \mathfrak{k}_{M}\right]\right)=\left(\left[\mathfrak{k}_{\sigma}, \mathfrak{k}_{\sigma}\right]\right), \quad \Phi(M) \cap \sigma \neq \varnothing, \quad \lambda \in\left\{\sigma+\varrho^{K_{\sigma}}\right\} .
$$

In $\S 6.3$, we give an example of a $\mathrm{SU}(3)$-manifold $M$ with generic stabilizer $\mathrm{SU}(2)$, and a $\operatorname{spin}^{c}$ bundle $\mathcal{S}$, where several $\sigma$ contribute to the multiplicity of a representation $\pi_{K \lambda}$ in $\mathcal{Q}_{K}(M, \mathcal{S})$. On Figure 2, the picture of the decomposition of $\mathcal{Q}_{K}(M, \mathcal{S})$ is given in terms of the representations $\mathrm{Q}_{K}^{\text {spin }}(\mathcal{P})$ associated with the $\mathrm{SU}(2)$-ancestors $\mathcal{P}$. All reduced spaces are points, but the multiplicity $\mathrm{Q}^{\operatorname{spin}}\left(M_{\mathcal{P}}\right)$ are equal to -1 , following from the orientation rule. On the picture, the links between admissible regular orbits $\mathcal{O}$ and their ancestors $\mathcal{P}$ are indicated by segments. We see that the trivial representation of $K$ has two ancestors $\mathcal{P}_{1}$ and $\mathcal{P}_{2}$ of type $(\mathfrak{h})$, so that the multiplicity of the trivial representation is equal to

$$
\mathrm{Q}^{\operatorname{spin}}\left(M_{\mathcal{P}_{1}}\right)+\mathrm{Q}^{\operatorname{spin}}\left(M_{\mathcal{P}_{2}}\right)=-2,
$$

and comes from two different faces of the Weyl chamber.

\subsection{Strategy}

The moment map $\Phi_{\mathcal{S}}$ permits us to define the Kirwan vector field $\varkappa_{\mathcal{S}}$ on $M$ : at $m \in M$, $\varkappa_{\mathcal{S}}$ is the tangent vector obtained by the infinitesimal action of $-\Phi_{\mathcal{S}}(m)$ at $m \in M$. Our 


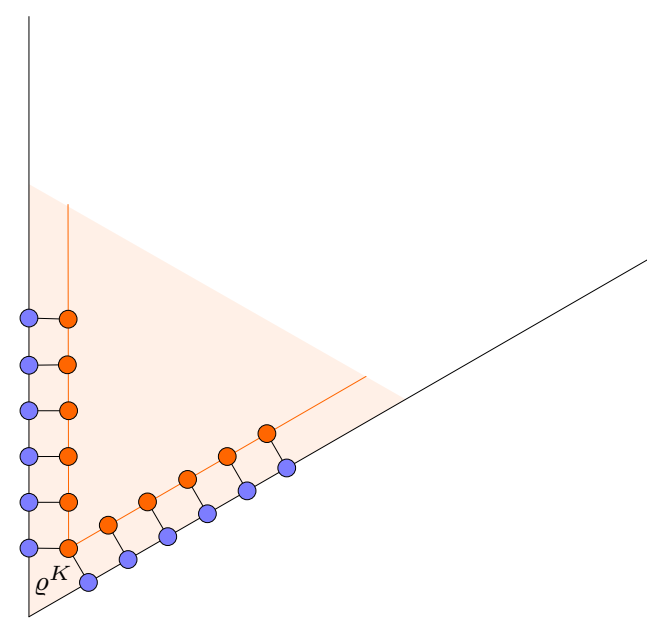

Figure 2. $K$-multiplicities and ancestors.

proof is based on a localization procedure using the vector field $\varkappa_{\mathcal{S}}$. Before going into the details, let us recall the genealogy of the method.

In [2], Atiyah and Bott calculate the cohomology of moduli spaces of vector bundles over Riemann surfaces by using a stratification defined by the Yang-Mills functional. This functional turns to the be the square of a moment map (in a infinite-dimensional setting). Their approach was developped by Kirwan in [20] to relate the cohomology of the Mumford GIT quotient with the equivariant cohomology of the initial manifold. Recall that in the symplectic setting the Kirwan vector field is the Hamiltonian vector field of the square of the moment map.

In [36], Witten proposed a non-abelian localisation procedure on the zero set of the Kirwan vector field for the integration of equivariant classes. This wonderful idea had a great influence in many other contexts. For example, Tian and Zhang [33] gives an analytical proof of the $[Q, R]=0$ theorem by deforming à la Witten the Dolbeault-Dirac operator with the Kirwan vector field.

In this paper we use a $\mathbf{K}$-theoretic analogue of the Witten non-abelian localization procedure. Let us briefly explain the main lines of this powerful tool which was initiated in [34], [35], [26] and developed in [28]. We use a topological deformation of the symbol of the Dirac operator $D$ by pushing the zero section of $\mathrm{T}^{*} M$ inside $\mathrm{T}^{*} M$ using the Kirwan vector field $\varkappa_{\mathcal{S}}$.

In Witten non-abelian localization formula, computation of integrals of equivariant cohomology classes on $M$ reduces to the study of contributions coming from a neighborhood of $Z_{\mathcal{S}}$, the set of zeros of the invariant vector field $\varkappa_{\mathcal{S}}$. Our $\mathbf{K}$-theoretical 
non-abelian localization formula allows us to compute the index $\mathcal{Q}_{K}(M, \mathcal{S})$ as a sum of equivariant indices of transversally elliptic operators associated with connected components $Z$ of $Z_{\mathcal{S}}$. We are able to identify them to some basic transversally elliptic symbols whose indices were computed by Atiyah-Singer (see [1]). Although these indices are infinite-dimensional representations, they are easier to understand than the original finite-dimensional representation $\mathcal{Q}_{K}(M, \mathcal{S})$. The main difficulty is in estimating which components $Z$ contributes to the $K$-invariant part. We are able to do so, using a miraculous estimate on distance between admissible coadjoint orbits proved in [29]. As shown by the final result, we have (in contrast to the Hamiltonian setting) to take in account several components and to identify their contributions.

\subsection{Outline of the article}

Let us explain the contents of the different sections of the article, and their main use in the final proof.

- In $\S 2$, we give the definition of the index of a $\operatorname{spin}^{c}$-bundle.

- In $\S 3$, we describe the canonical spin $^{c}$-bundle on admissible coadjoint orbits (see (3.12)). For a $K$-admissible coadjoint orbit $\mathcal{P}$, we determine the regular admissible orbit $\mathcal{O}$ such that if $\mathrm{Q}_{K}^{\text {spin }}(\mathcal{P})$ is not zero, then $\mathrm{Q}_{K}^{\text {spin }}(\mathcal{P})=\pi_{\mathcal{O}}$ (Proposition 3.6).

In Proposition 3.14, we state the "magical inequality" that will be used over and over again in this article.

- In $\S 4$, we define the Witten deformation and recall some of its properties (proved in [26], [28]). It allows us to reduce the computation of $\mathcal{Q}_{K}(M, \mathcal{S})$ to indices $q_{Z}$ of simpler transversally elliptic operators defined in neighborhoods of connected components of $Z_{\mathcal{S}}=\left\{\varkappa_{\mathcal{S}}=0\right\}$.

We introduce a function $d_{\mathcal{S}}: Z_{\mathcal{S}} \rightarrow \mathbb{R}$. If $d_{\mathcal{S}}$ takes strictly positive value on some component $Z$ of $Z_{\mathcal{S}}$, then the $K$-invariant part of the (virtual) representation $q_{Z}$ is equal to zero (Proposition 4.17). This is a very important technical proposition.

If $\mathcal{O}$ is an admissible regular coadjoint orbit, the shifting trick leads us to study the manifold $M \times \mathcal{O}^{*}$ with $\operatorname{spin}^{c}$-bundle $\mathcal{S} \otimes \mathcal{S}_{\mathcal{O}^{*}}$. We want to select the component $Z$ of $Z_{\mathcal{S} \otimes \mathcal{S}_{\mathcal{O}^{*}}}$, so that $\left[q_{Z}\right]^{K}$ is not zero.

Here is where we discover that, for $\mathcal{Q}_{K}(M, \mathcal{S})$ to be non zero, it is necessary that the semi-simple part of the generic stabilizer $\left(\mathfrak{k}_{M}\right)$ of the action of $K$ on $M$ is equal to the semi-simple part of a Levi subalgebra $(\mathfrak{h})$ of $\mathfrak{k}$. It follows that such a component $Z$ is described rather simply as an induced manifold $K \times{ }_{H}(Y \times o(\mathfrak{h}))$, where $Y$ is a $H /[H, H]$ manifold, and $o(\mathfrak{h})$ is the $[H, H]$-orbit of the corresponding $\varrho^{[H, H]}$ element. Then we use the fact that the quantization of the orbit of $\varrho$ is the trivial representation. In fact, to 
determine the contributing components $Z$, we study a function $d_{\mathcal{O}}: Z_{\mathcal{S} \otimes \mathcal{S}_{\mathcal{O}^{*}}} \rightarrow \mathbb{R}$ relating the representation of $K_{m}$ on $\mathrm{T}_{m} M$ and the norm of $\Phi_{\mathcal{S}}(m)$. Here $K_{m}$ is the stabilizer of $m \in M$. It relies on the "magical inequality" (Proposition 3.14) on distance of regular weights to faces of the Weyl chamber proved in [29].

- In $\S 5$, we explain how to define indices on singular reduced spaces. The main theorem is their invariance under small deformation. We then have done all the work needed to be able to prove the main theorem.

- The last section is dedicated to some simple examples intended to show several features of our theory.

\section{Acknowledgments}

We wish to thank a referee for his useful remarks. We wish to thank the Research in Pairs program at Mathematisches Forschungsinstitut Oberwolfach (February 2014), where this work was started. The second author wish to thank Michel Duflo for many discussions.

\section{Notation}

Throughout the paper:

- $K$ denotes a compact connected Lie group with Lie algebra $\mathfrak{k}$.

- $T$ is a maximal torus in $K$ with Lie algebra $t$.

- $\Lambda \subset \mathfrak{t}^{*}$ is the weight lattice of $T$ : every element $\mu \in \Lambda$ defines a 1-dimensional $T$ representation, denoted $\mathbb{C}_{\mu}$, where $t=\exp (X)$ acts by $t^{\mu}:=e^{i\langle\mu, X\rangle}$.

- We fix a $K$-invariant inner product $(\cdot, \cdot)$ on $\mathfrak{k}$. This allows us to identify $\mathfrak{k}$ and $\mathfrak{k}^{*}$ when needed.

We denote by $\langle\cdot, \cdot\rangle$ the natural duality between $\mathfrak{k}$ and $\mathfrak{k}^{*}$.

- We denote by $R(K)$ the representation ring of $K$ : an element $E \in R(K)$ can be represented as finite sum $E=\sum_{\mu \in \widehat{K}} \mathrm{~m}_{\mu} \pi_{\mu}$, with $\mathrm{m}_{\mu} \in \mathbb{Z}$. The multiplicity of the trivial representation is denoted by $[E]^{K}$.

- We denote by $\widehat{R}(K)$ the space of $\mathbb{Z}$-valued functions on $\widehat{K}$. An element $E \in \widehat{R}(K)$ can be represented as an infinite sum $E=\sum_{\mu \in \widehat{K}} \mathrm{~m}(\mu) V_{\mu}$, with $\mathrm{m}(\mu) \in \mathbb{Z}$.

- If $H$ is a closed subgroup of $K$, the induction map $\operatorname{Ind}_{H}^{K}: \widehat{R}(H) \rightarrow \widehat{R}(K)$ is the dual of the restriction morphism $R(K) \rightarrow R(H)$.

- When $K$ acts on a set $X$, the stabilizer subgroup of $x \in X$ is denoted by

$$
K_{x}:=\{k \in K: k \cdot x=x\} .
$$

The Lie algebra of $K_{x}$ is denoted by $\mathfrak{k}_{x}$. 
- An element $\xi \in \mathfrak{k}^{*}$ is called regular if $K_{\xi}$ is a maximal torus of $K$.

- When $K$ acts on a manifold $M$, we denote

$$
X_{M}(m):=\left.\frac{d}{d t}\right|_{t=0} e^{-t X} \cdot m
$$

the vector field generated by $-X \in \mathfrak{k}$. Sometimes we will also use the notation

$$
X_{M}(m)=-X \cdot m \text {. }
$$

The set of zeros of the vector field $X_{M}$ is denoted $M^{X}$.

- If $V$ is a complex (ungraded) vector space, then the exterior space

$$
\bigwedge V=\bigwedge^{+} V \oplus \bigwedge^{-} V
$$

will be $\mathbb{Z} / 2 \mathbb{Z}$ graded in even and odd elements.

- If $E_{1}=E_{1}^{+} \oplus E_{1}^{-}$and $E_{2}=E_{2}^{+} \oplus E_{2}^{-}$are two $\mathbb{Z} / 2 \mathbb{Z}$ graded vector spaces (or vector bundles), the tensor product $E_{1} \otimes E_{2}$ is $\mathbb{Z} / 2 \mathbb{Z}$-graded with

$$
\left(E_{1} \otimes E_{2}\right)^{+}=E_{1}^{+} \otimes E_{2}^{+} \oplus E_{1}^{-} \otimes E_{2}^{-} \quad \text { and } \quad\left(E_{1} \otimes E_{2}\right)^{-}=E_{1}^{-} \otimes E_{2}^{+} \oplus E_{1}^{+} \otimes E_{2}^{-} .
$$

Similarly, the spaces $\operatorname{End}\left(E_{i}\right)$ are $\mathbb{Z} / 2 \mathbb{Z}$ graded. The action of $\operatorname{End}\left(E_{1}\right) \otimes \operatorname{End}\left(E_{2}\right)$ on $E_{1} \otimes E_{2}$ obeys the usual sign rules: for example, if $f \in \operatorname{End}\left(E_{2}\right)^{-}, v_{1} \in E_{1}^{-}$and $v_{2} \in E_{2}$, then $f\left(v_{1} \otimes v_{2}\right)=-v_{1} \otimes f v_{2}$.

- If $E$ is a vector space and $M$ a manifold, we denote by $[E]$ the trivial vector bundle on $M$ with fiber $E$.

\section{Spin $^{c}$ equivariant index}

\subsection{Spin $^{c}$ modules}

Let $V$ be an oriented Euclidean space of even dimension $n=2 \ell$. We denote by $\mathrm{Cl}(V)$ its Clifford algebra. If $e_{1}, \ldots, e_{n}$ is an oriented orthonormal frame of $V$, we define the element

$$
\varepsilon:=(i)^{\ell} e_{1} \ldots e_{n} \in \mathrm{Cl}(V) \otimes \mathbb{C}
$$

that depends only of the orientation. We have $\varepsilon^{2}=1$ and $\varepsilon v=-v \varepsilon$ for any $v \in V$.

If $E$ is a complex $\mathrm{Cl}(V)$-module, the Clifford map is denoted $\mathbf{c}_{E}: \mathrm{Cl}(V) \rightarrow \operatorname{End}(E)$. We see then that the order-2 element $\varepsilon_{E}:=\mathbf{c}_{E}(\varepsilon)$ defines a $\mathbb{Z} / 2 \mathbb{Z}$-graduation on $E$ by defining $E^{ \pm}:=\operatorname{ker}\left(\operatorname{Id}_{E} \mp \varepsilon_{E}\right)$. Moreover, the maps $\mathbf{c}_{E}(v): E \rightarrow E$ for $v \in V$ interchange the subspaces $E^{+}$and $E^{-}$. This graduation will be called the canonical graduation of the Clifford module $E$.

We follow the conventions of [4]. Recall the following fundamental fact. 
Proposition 2.1. Let $V$ be an even-dimensional Euclidean space.

- There exists a complex $\mathrm{Cl}(V)$-module $S$ such that the Clifford morphism

$$
\mathbf{c}_{S}: \mathrm{Cl}(V) \longrightarrow \operatorname{End}(S)
$$

induces an isomorphism of complex algebra $\mathrm{Cl}(V) \otimes \mathbb{C} \simeq \operatorname{End}(S)$.

- The Clifford module $S$ is unique up to isomorphism. We call it the spinor $\mathrm{Cl}(V)$ module.

- Any complex $\mathrm{Cl}(V)$-module $E$ has the following decomposition

$$
E \simeq S \otimes \operatorname{hom}_{\mathrm{Cl}(V)}(S, E)
$$

where $\operatorname{hom}_{\mathrm{Cl}(V)}(S, E)$ is the vector space spanned by the $\mathrm{Cl}(V)$-complex linear maps from $S$ to $E$. If $V$ is oriented and the Clifford modules $S$ and $E$ carry their canonical grading, then (2.4) is an isomorphism of graded Clifford $\mathrm{Cl}(V)$-modules.

Let $V=V_{1} \oplus V_{2}$ be an orthogonal decomposition of even-dimensional Euclidean spaces. We choose an orientation $o\left(V_{1}\right)$ on $V_{1}$. There is a one-to-one correspondence between the graded $\mathrm{Cl}\left(V_{2}\right)$-modules and the graded $\mathrm{Cl}(V)$-modules defined as follows. Let $S_{1}$ be the spinor module for $\mathrm{Cl}\left(V_{1}\right)$. If $W$ is a $\mathrm{Cl}\left(V_{2}\right)$-module, the vector space $E:=S_{1} \otimes W$ is a $\mathrm{Cl}(V)$-module with the Clifford map defined by

$$
\mathbf{c}_{E}\left(v_{1} \oplus v_{2}\right):=\mathbf{c}_{S_{1}}\left(v_{1}\right) \otimes \operatorname{Id}_{W}+\varepsilon_{S_{1}} \otimes \mathbf{c}_{W}\left(v_{2}\right) .
$$

Here $v_{i} \in V_{i}$ and $\varepsilon_{S_{1}} \in \operatorname{End}\left(S_{1}\right)$ defines the canonical graduation of $S_{1}$. Conversely, if $E$ is a graded $\mathrm{Cl}(V)$-module, the vector space $W:=\operatorname{hom}_{\mathrm{Cl}\left(V_{1}\right)}\left(S_{1}, E\right)$ formed by the complex linear maps $f: S_{1} \rightarrow E$ commuting with the action of $\mathrm{Cl}\left(V_{1}\right)$ has a natural structure of $\mathrm{Cl}\left(V_{2}\right)$ graded module and $E \simeq S_{1} \otimes W$.

If we fix an orientation $o(V)$ on $V$, it fixes an orientation $o\left(V_{2}\right)$ on $V_{2}$ by the relation $o(V)=o\left(V_{1}\right) o\left(V_{2}\right)$. Then the Clifford modules $E$ and $W$ carries their canonical $\mathbb{Z} / 2 \mathbb{Z}$ graduation, and $E \simeq S_{1} \otimes W$ becomes an identity of graded Clifford modules.

Example 2.2. Let $H$ be a Euclidean vector space equipped with a complex structure $J \in O(H)$ : we consider the complex vector space $\bigwedge_{J} H$. Denote by $m(v)$ the exterior multiplication by $v$. The action $\mathbf{c}$ of $H$ on $\bigwedge_{J} H$ given by $\mathbf{c}(v)=m(v)-m(v)^{*}$ satisfies $\mathbf{c}(v)^{2}=-\|v\|^{2} \mathrm{Id}$. Thus, $\bigwedge_{J} H$, equipped with the action $\mathbf{c}$, is a realization of the spinor module for $H$. Note that the group $\mathrm{U}(J)$ of unitary transformations of $H$ acts naturally on $\bigwedge_{J} H$. If one choose the orientation on $H$ induced by the complex structure, one sees that the canonical grading is $\left(\bigwedge_{J} H\right)^{ \pm}=\bigwedge_{J}^{ \pm} H$. 
Suppose now that we have another complex structure $J^{\prime} \in O(H)$ : the vector space $\bigwedge_{J^{\prime}} H$ is another spinor module for $H$. We denote by $\varepsilon_{J}^{J^{\prime}}$ the ratio between the orientations defined by $J$ and $J^{\prime}$. One can check that

$$
\bigwedge_{J^{\prime}} H \simeq \varepsilon_{J}^{J^{\prime}} \mathbb{C}_{\chi} \otimes \bigwedge_{J} H
$$

as a graded $\mathrm{Cl}(H)$-module and also as a graded $\mathrm{U}\left(J^{\prime}\right) \cap \mathrm{U}(J)$-module. Here $\mathbb{C}_{\chi}$ is the 1dimensional representation of $\mathrm{U}\left(J^{\prime}\right) \cap \mathrm{U}(J)$ associated with the unique character $\chi$ defined by the relation $\chi(g)^{2}=\operatorname{det}_{J^{\prime}}(g) \operatorname{det}_{J}(g)^{-1}$, for all $g \in \mathrm{U}\left(J^{\prime}\right) \cap \mathrm{U}(J)$.

Example 2.3. When $V=Q \oplus Q$, with $Q$ being a Euclidean space, we can identify $V$ with $Q_{\mathbb{C}}$ by $(x, y) \mapsto x \oplus i y$. Thus $S_{Q}:=\bigwedge Q_{\mathbb{C}}$ is a realization of the spinor module for $V$. It carries a natural action of the orthogonal group $\mathrm{O}(Q)$ acting diagonally. If $Q$ carries a complex structure $J \in O(Q)$, we can consider the spin modules $\bigwedge_{J} Q$ and $\bigwedge_{-J} Q$ for $Q$. We have then the isomorphism $S_{Q} \simeq \bigwedge_{J} Q \otimes \bigwedge_{-J} Q$ of graded $\mathrm{Cl}(V)$-modules (it is also an isomorphism of $\mathrm{U}(J)$-modules).

\subsection{Spin $^{c}$ structures}

Consider now the case of a Euclidean vector bundle $\mathcal{V} \rightarrow M$ of even rank. Let $\mathrm{Cl}(\mathcal{V}) \rightarrow M$ be the associated Clifford algebra bundle. A complex vector bundle $\mathcal{E} \rightarrow M$ is a $\mathrm{Cl}(\mathcal{V})$ module if there is a bundle algebra morphism $\mathbf{c}_{\mathcal{E}}: \mathrm{Cl}(\mathcal{V}) \rightarrow \operatorname{End}(\mathcal{E})$.

Definition 2.4. Let $\mathcal{S} \rightarrow M$ be a $\mathrm{Cl}(\mathcal{V})$-module such that the map $\mathbf{c}_{\mathcal{S}}$ induces an isomorphism $\operatorname{Cl}(\mathcal{V}) \otimes_{\mathbb{R}} \mathbb{C} \rightarrow \operatorname{End}(\mathcal{S})$. Then we say that $\mathcal{S}$ is a $\operatorname{spin}^{c}$-bundle for $\mathcal{V}$.

As in the linear case, an orientation on the vector bundle $\mathcal{V}$ determines a $\mathbb{Z} / 2 \mathbb{Z}$ grading of the vector bundle $\mathcal{S}$ (called the canonical graduation) such that for any $v \in \mathcal{V}_{m}$, the linear $\operatorname{map}\left({ }^{1}\right) \mathbf{c}_{\mathcal{S}}(m, v):\left.\left.\mathcal{S}\right|_{m} \rightarrow \mathcal{S}\right|_{m}$ is odd.

Example 2.5. When $\mathcal{H} \rightarrow M$ is a Hermitian vector bundle, the complex vector bundle $\bigwedge \mathcal{H}$ is a $\operatorname{spin}^{c}$ bundle for $\mathcal{H}$. If one choose the orientation of the vector bundle $\mathcal{H}$ induced by the complex structure, one sees that the canonical grading is $(\bigwedge \mathcal{H})^{ \pm}=\bigwedge^{ \pm} \mathcal{H}$.

We assume that the vector bundle $\mathcal{V}$ is oriented, and we consider two $\operatorname{spin}^{c}$-bundles $\mathcal{S}$ and $\mathcal{S}^{\prime}$ for $\mathcal{V}$, both with their canonical grading. We have the following identity of graded spin ${ }^{c}$-bundles: $\mathcal{S}^{\prime} \simeq \mathcal{S} \otimes \mathbb{L}_{\mathcal{S}, \mathcal{S}^{\prime}}$, where $\mathbb{L}_{\mathcal{S}, \mathcal{S}^{\prime}}$ is a complex line bundle on $M$ defined by the relation

$$
\mathbb{L}_{\mathcal{S}, \mathcal{S}^{\prime}}:=\operatorname{hom}_{\mathrm{Cl}(\mathcal{V})}\left(\mathcal{S}, \mathcal{S}^{\prime}\right)
$$

$\left.{ }^{1}\right)$ The map $\mathbf{c}_{\mathcal{S}}(m, \cdot):\left.\mathcal{V}\right|_{m} \rightarrow \operatorname{End}\left(\left.\mathcal{S}\right|_{m}\right)$ will also be denoted by $\mathbf{c}_{\left.\mathcal{S}\right|_{m}}$. 
Definition 2.6. Let $\mathcal{V} \rightarrow M$ be a Euclidean vector bundle of even rank. The determinant line bundle of a $\operatorname{spin}^{c}$-bundle $\mathcal{S}$ on $\mathcal{V}$ is the line bundle $\operatorname{det}(\mathcal{S}) \rightarrow M$ defined by the relation

$$
\operatorname{det}(\mathcal{S}):=\operatorname{hom}_{\mathrm{Cl}(\mathcal{V})}(\overline{\mathcal{S}}, \mathcal{S})
$$

where $\overline{\mathcal{S}}$ is the $\mathrm{Cl}(\mathcal{V})$-module with opposite complex structure.

Example 2.7. When $\mathcal{H} \rightarrow M$ is a Hermitian vector bundle, the determinant line bundle of the $\operatorname{spin}^{c}$-bundle $\bigwedge \mathcal{H}$ is $\operatorname{det}(\mathcal{H}):=\bigwedge^{\max } \mathcal{H}$.

If $\mathcal{S}$ and $\mathcal{S}^{\prime}$ are two $\operatorname{spin}^{c}$-bundles for $\mathcal{V}$, we see that

$$
\operatorname{det}\left(\mathcal{S}^{\prime}\right)=\operatorname{det}(\mathcal{S}) \otimes \mathbb{L}_{\mathcal{S}, \mathcal{S}^{\prime}}^{\otimes 2}
$$

Assume that $\mathcal{V}=\mathcal{V}_{1} \oplus \mathcal{V}_{2}$ is an orthogonal sum of Euclidean vector bundles of even rank. We assume that $\mathcal{V}_{1}$ is oriented, and let $\mathcal{S}_{1}$ be a spin ${ }^{c}$-bundle for $\mathcal{V}_{1}$ that we equip with its canonical grading. If $\mathcal{E}$ is a Clifford bundle for $\mathcal{V}$, then we have the following isomorphism $\left({ }^{2}\right)$

$$
\mathcal{E} \simeq \mathcal{S}_{1} \otimes \mathcal{W}
$$

where $\mathcal{W}:=\operatorname{hom}_{\mathrm{Cl}\left(\mathcal{V}_{1}\right)}\left(\mathcal{S}_{1}, \mathcal{E}\right)$ is a Clifford bundle for $\mathcal{V}_{2}$. If $\mathcal{V}$ is oriented, it fixes an orientation $o\left(\mathcal{V}_{2}\right)$ on $\mathcal{V}_{2}$ by the relation $o(\mathcal{V})=o\left(\mathcal{V}_{1}\right) o\left(\mathcal{V}_{2}\right)$. Then the Clifford modules $\mathcal{E}$ and $\mathcal{W}$ carry their canonical $\mathbb{Z} / 2 \mathbb{Z}$ grading, and (2.7) becomes an identity of graded Clifford modules.

In the particular situation when $\mathcal{S}$ is a $\operatorname{spin}^{c}$-bundle for $\mathcal{V}$, then $\mathcal{S} \simeq \mathcal{S}_{1} \otimes \mathcal{S}_{2}$, where $\mathcal{S}_{2}:=\operatorname{hom}_{\mathrm{Cl}\left(\mathcal{V}_{1}\right)}\left(\mathcal{S}_{1}, \mathcal{S}\right)$ is a $\operatorname{spin}^{c}$-bundle for $\mathcal{V}_{2}$. At the level of determinant line bundles, we obtain $\operatorname{det}(\mathcal{S})=\operatorname{det}\left(\mathcal{S}_{1}\right) \otimes \operatorname{det}\left(\mathcal{S}_{2}\right)$.

Let us end this section by recalling the notion of spin-structure and $\operatorname{spin}^{c}$-structure. Let $\mathcal{V} \rightarrow M$ be an oriented Euclidean vector bundle of rank $n$, and let $\mathrm{P}_{\mathrm{SO}}(\mathcal{V})$ be its orthogonal frame bundle: it is a principal $\mathrm{SO}_{n}$ bundle over $M$.

Let us consider the spinor group $\operatorname{spin}_{n}$ which is the double cover of the group $\mathrm{SO}_{n}$. The group $\operatorname{spin}_{n}$ is a subgroup of the group $\operatorname{spin}_{n}^{c}$ which covers $\mathrm{SO}_{n}$ with fiber $U(1)$.

A spin structure on $\mathcal{V}$ is a $\operatorname{spin}_{n}$-principal bundle $\mathrm{P}_{\text {spin }}(\mathcal{V})$ over $M$ together with a $\operatorname{spin}_{n}$-equivariant map $\mathrm{P}_{\text {spin }}(\mathcal{V}) \rightarrow \mathrm{P}_{S O}(\mathcal{V})$.

We assume now that $\mathcal{V}$ is of even rank $n=2 \ell$. Let $\mathrm{S}_{n}$ be the irreducible complex spin representation of $\operatorname{spin}_{n}$. Recall that $\mathrm{S}_{n}=\mathrm{S}_{n}^{+} \oplus \mathrm{S}_{n}^{-}$inherits a canonical Clifford action $\mathbf{c}: \mathbb{R}^{n} \rightarrow \operatorname{End}\left(\mathrm{S}_{n}\right)$, which is $\operatorname{spin}_{n}$-equivariant, and which interchanges the graduation: $\mathbf{c}(v): \mathrm{S}_{n}^{ \pm} \rightarrow \mathrm{S}_{n}^{\mp}$. The spinor bundle attached to the spin-structure $\mathrm{P}_{\text {spin }}(\mathcal{V})$ is

$$
\mathcal{S}:=\mathrm{P}_{\text {spin }}(\mathcal{V}) \times_{\operatorname{spin}_{n}} \mathrm{~S}_{n}
$$

$\left({ }^{2}\right)$ The proof is identical to the linear case explained earlier. 
A $\operatorname{spin}^{c}$-bundle for $\mathcal{V}$ determines a $\operatorname{spin}^{c}$ structure, that is a principal bundle over $M$ with structure group $\operatorname{spin}_{n}^{c}$. When $\mathcal{V}$ admits a spin-structure, any $\operatorname{spin}^{c}$-bundle for $\mathcal{V}$ is of the form $\mathcal{S}_{L}=\mathcal{S}_{\text {spin }} \otimes L$ where $\mathcal{S}_{\text {spin }}$ is the spinor bundle attached to the spin-structure and $L$ is a line bundle on $M$. Then the determinant line bundle for $\mathcal{S}_{L}$ is $L^{\otimes 2}$.

\subsection{Moment maps and Kirwan vector field}

In this section, we consider the case of a Riemannian manifold $M$ acted on by a compact Lie group $K$. Let $\mathcal{S} \rightarrow M$ be a $\operatorname{spin}^{c}$-bundle on $M$. If the $K$-action lifts to the spin ${ }^{c}$-bundle $\mathcal{S}$ in such a way that the bundle map $c_{\mathcal{S}}: \mathrm{Cl}(\mathrm{T} M) \rightarrow \operatorname{End}(\mathcal{S})$ commutes with the $K$-action, we say that $\mathcal{S}$ defines a $K$-equivariant $\operatorname{spin}^{c}$-bundle on $M$. In this case, the $K$-action lifts also to the determinant line bundle $\operatorname{det}(\mathcal{S})$. The choice of an invariant Hermitian connection $\nabla$ on $\operatorname{det}(\mathcal{S})$ determines an equivariant map $\Phi_{\mathcal{S}}: M \rightarrow \mathfrak{k}^{*}$ and a 2 -form $\Omega_{\mathcal{S}}$ on $M$ by means of the Kostant relations

$$
\mathcal{L}(X)-\nabla_{X_{M}}=2 i\left\langle\Phi_{\mathcal{S}}, X\right\rangle \quad \text { and } \quad \nabla^{2}=-2 i \Omega_{\mathcal{S}}
$$

for every $X \in \mathfrak{k}$. Here $\mathcal{L}(X)$ denotes the infinitesimal action on the sections of $\operatorname{det}(\mathcal{S})$. We will say that $\Phi_{\mathcal{S}}$ is the moment map for $\mathcal{S}$ (it depends however of the choice of a connection).

Via the equivariant Bianchi formula, (2.8) induce the relations

$$
\iota\left(X_{M}\right) \Omega_{\mathcal{S}}=-d\left\langle\Phi_{\mathcal{S}}, X\right\rangle \text { and } d \Omega_{\mathcal{S}}=0
$$

for every $X \in \mathfrak{k}$.

In particular the function $m \rightarrow\left\langle\Phi_{\mathcal{S}}(m), X\right\rangle$ is locally constant on $M^{X}$.

Remark 2.8. Let $b \in \mathfrak{k}$ and $m \in M^{b}$, the set of zeros of $b_{M}$. We consider the linear actions $\left.\mathcal{L}(b)\right|_{\mathcal{S}_{m}}$ and $\left.\mathcal{L}(b)\right|_{\operatorname{det}(\mathcal{S})_{m}}$ on the fibers at $m$ of the $\operatorname{spin}^{c}$-bundle $\mathcal{S}$ and the line bundle $\operatorname{det}(\mathcal{S})$. Kostant relations imply $\left.\mathcal{L}(b)\right|_{\operatorname{det}(\mathcal{S})_{m}}=2 i\left\langle\Phi_{\mathcal{S}}(m), b\right\rangle$. The irreducibility of $\mathcal{S}$ implies that

$$
\left.\mathcal{L}(b)\right|_{\mathcal{S}_{m}}=i\left\langle\Phi_{\mathcal{S}}(m), b\right\rangle \operatorname{Id}_{\mathcal{S}_{m}} .
$$

Furthermore, the function $m \mapsto\left\langle\Phi_{\mathcal{S}}(m), b\right\rangle$ is locally constant on $M^{b}$.

Remark 2.9. Notice that

- The closed equivariant form $\Omega_{\mathcal{S}}-\left\langle\Phi_{\mathcal{S}}, X\right\rangle$ represents half of the equivariant first Chern class of the line bundle $\operatorname{det}(\mathcal{S})$.

- In general, the closed 2 -form $\Omega_{\mathcal{S}}$ is not symplectic. Furthermore, if we take any (real valued) invariant 1 -form $A$ on $M, \nabla+i A$ is another connection on $\operatorname{det}(\mathcal{S})$. The corresponding curvature and moment map will be modified in $\Omega_{\mathcal{S}}-\frac{1}{2} d A$ and $\Phi_{\mathcal{S}}-\frac{1}{2} \Phi_{A}$, where $\Phi_{A}: M \rightarrow \mathfrak{k}^{*}$ is defined by the relation $\left\langle\Phi_{A}, X\right\rangle=-\iota\left(X_{M}\right) A$. 
Let $\Phi: M \rightarrow \mathfrak{k}$ be a $K$ equivariant map. We define the $K$-invariant vector field $\varkappa_{\Phi}$ on $M$ by

$$
\varkappa_{\Phi}(m):=-\Phi(m) \cdot m
$$

and we call it the Kirwan vector field associated with $\Phi$. The set where $\varkappa_{\Phi}$ vanishes is a K-invariant subset that we denote by $Z_{\Phi} \subset M$.

We identify $\mathfrak{k}^{*}$ to $\mathfrak{k}$ by our choice of $K$-invariant scalar product and we will have a particular interest in the equivariant map $\Phi_{\mathcal{S}}: M \rightarrow \mathfrak{k}^{*} \simeq \mathfrak{k}$ associated with the spin $^{c}$ bundle $\mathcal{S}$. In this case we may denote the $K$-invariant vector field $\varkappa_{\Phi_{\mathcal{S}}}$ simply by $\varkappa_{\mathcal{S}}$ (even if it depends of the choice of a connection):

$$
\varkappa_{\mathcal{S}}(m):=-\Phi_{\mathcal{S}}(m) \cdot m
$$

and we denote $Z_{\Phi_{\mathcal{S}}}$ by $Z_{\mathcal{S}}$.

As $\Phi_{\mathcal{S}}$ is a moment map, we have the following basic description (see [26] and [28]).

LEMMA 2.10. If the manifold $M$ is compact, the set $\Phi_{\mathcal{S}}\left(Z_{\mathcal{S}}\right)$ is a finite collection of coadjoint orbits. For any coadjoint orbit $\mathcal{O}=K \beta$, we have

$$
Z_{\mathcal{S}} \cap \Phi_{\mathcal{S}}^{-1}(\mathcal{O})=K\left(M^{\beta} \cap \Phi_{\mathcal{S}}^{-1}(\beta)\right)
$$

Here we have identified $\beta \in \mathfrak{k}^{*}$ to an element in $\mathfrak{k}$ still denoted by $\beta$.

Remark 2.11. A small computation gives $\frac{1}{2} d\left\|\Phi_{\mathcal{S}}\right\|^{2}=-\iota\left(\varkappa_{\mathcal{S}}\right) \Omega_{\mathcal{S}}$, hence the zeros of $\varkappa_{\mathcal{S}}$ are critical points of $\left\|\Phi_{\mathcal{S}}\right\|^{2}$.

Remark 2.12. From the previous remark, we notice that any $\beta$ in the image $\Phi_{\mathcal{S}}\left(Z_{\mathcal{S}}\right)$ is such that $\|\beta\|^{2}$ is a critical value of the map $\left\|\Phi_{\mathcal{S}}\right\|^{2}$. Although the map $\Phi_{\mathcal{S}}$, as well as the set, $Z_{\mathcal{S}}$ vary when we vary the connection, we see that the image $\Phi_{\mathcal{S}}\left(Z_{\mathcal{S}}\right)$ is contained in a finite set of coadjoint orbits that does not depend of the connection (see [28]).

Figure 3 describes the set $\Phi_{\mathcal{S}}\left(Z_{\mathcal{S}}\right)$ for the action of the diagonal torus of $K=\mathrm{SU}(3)$ on the orbit $K \varrho$ equipped with its canonical $\operatorname{spin}^{c}$-bundle.

\subsection{Equivariant index}

Assume in this section that the Riemannian $K$-manifold $M$ is compact, even-dimensional, oriented, and equipped with a $K$-equivariant $\operatorname{spin}^{c}$-bundle $\mathcal{S} \rightarrow M$. The orientation induces a decomposition $\mathcal{S}=\mathcal{S}^{+} \oplus \mathcal{S}^{-}$, and the corresponding $\operatorname{spin}^{c}$ Dirac operator is a first-order elliptic operator $\mathcal{D}_{\mathcal{S}}: \Gamma\left(M, \mathcal{S}^{+}\right) \rightarrow \Gamma\left(M, \mathcal{S}^{-}\right)$[4], [9]. Its principal symbol is the bundle map $\sigma(M, \mathcal{S}) \in \Gamma\left(\mathrm{T}^{*} M, \operatorname{hom}\left(p^{*} \mathcal{S}^{+}, p^{*} \mathcal{S}^{-}\right)\right)$defined by the relation

$$
\sigma(M, \mathcal{S})(m, \nu)=\mathbf{c}_{\left.\mathcal{S}\right|_{m}}(\tilde{\nu}):\left.\left.\mathcal{S}\right|_{m} ^{+} \longrightarrow \mathcal{S}\right|_{m} ^{-}
$$




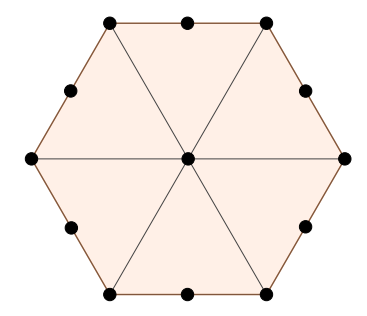

Figure 3. The set $\Phi_{\mathcal{S}}\left(Z_{\mathcal{S}}\right)$

Here $\nu \in \mathrm{T}^{*} M \mapsto \tilde{\nu} \in \mathrm{T} M$ is the identification defined by the Riemannian structure.

If $\mathcal{W} \rightarrow M$ is a complex $K$-vector bundle, we can define similarly the twisted Dirac operator $\mathcal{D}_{\mathcal{S}}^{\mathcal{W}}: \Gamma\left(M, \mathcal{S}^{+} \otimes \mathcal{W}\right) \rightarrow \Gamma\left(M, \mathcal{S}^{-} \otimes \mathcal{W}\right)$.

Definition 2.13. Let $\mathcal{S} \rightarrow M$ be an equivariant $\operatorname{spin}^{c}$-bundle. We denote

- by $\mathcal{Q}_{K}(M, \mathcal{S}) \in R(K)$ the equivariant index of the operator $\mathcal{D}_{\mathcal{S}}$,

- by $\mathcal{Q}_{K}(M, \mathcal{S} \otimes \mathcal{W}) \in R(K)$ the equivariant index of the operator $\mathcal{D}_{\mathcal{S}}^{\mathcal{W}}$.

Let $\hat{A}(M)(X)$ be the equivariant $\hat{\mathrm{A}}$-genus class of $M$ : it is an equivariant analytic function from a neighborhood of $0 \in \mathfrak{k}$ with value in the algebra of differential forms on $M$. Berline-Vergne equivariant index formula [4, Theorem 8.2] asserts that

$$
\mathcal{Q}_{K}(M, \mathcal{S})\left(e^{X}\right)=\left(\frac{i}{2 \pi}\right)^{(\operatorname{dim} M) / 2} \int_{M} e^{-i\left(\Omega_{\mathcal{S}}-\left\langle\Phi_{\mathcal{S}}, X\right\rangle\right)} \hat{A}(M)(X)
$$

for $X \in \mathfrak{k}$ small enough. Here we write $\mathcal{Q}_{K}(M, \mathcal{S})\left(e^{X}\right)$ for the trace of the element $e^{X} \in K$ in the virtual representation $\mathrm{Q}_{K}(M, \mathcal{S})$ of $K$. It shows in particular that $\mathrm{Q}_{K}(M, \mathcal{S}) \in$ $R(K)$ is a topological invariant: it only depends of the class of the equivariant form $\Omega_{\mathcal{S}}-\left\langle\Phi_{\mathcal{S}}, X\right\rangle$, which represents half of the first equivariant Chern class of the line bundle $\operatorname{det}(\mathcal{S})$.

Example 2.14. We consider the simplest case of the theory. Let $M:=\mathbb{P}^{1}(\mathbb{C})$ be the projective space of (complex) dimension 1 . We write an element of $M$ as $\left[z_{1}, z_{2}\right]$ in homogeneous coordinates. Consider the (ample) line bundle $\mathcal{L} \rightarrow \mathbb{P}^{1}$, dual of the tautological bundle. Let $\mathcal{S}(n)$ be the $\operatorname{spin}^{c}$-bundle $\bigwedge_{\mathbb{C}} \mathrm{T} M \otimes \mathcal{L}^{\otimes n}$. The character $\mathcal{Q}_{T}(M, \mathcal{S}(n))$ is equal to $H^{0}\left(\mathbb{P}^{1}, \mathcal{O}(n)\right)-H^{1}\left(\mathbb{P}^{1}, \mathcal{O}(n)\right)$, where $\mathcal{O}(n)$ is the sheaf of holomorphic section of $\mathcal{L}^{\otimes n}$. Then, for $n \geqslant 0$,

$$
\mathcal{Q}_{T}(M, \mathcal{S}(n))=\sum_{k=0}^{n} t^{k}
$$

Here $T=\{t \in \mathbb{C}:|t|=1\}$ acts on $\left[z_{1}, z_{2}\right]$ via $t \cdot\left[z_{1}, z_{2}\right]=\left[t^{-1} z_{1}, z_{2}\right]$. 


\section{Coadjoint orbits}

In this section, we describe $\operatorname{spin}^{c}$-bundles on admissible coadjoint orbits of $K$ and the equivariant indices of the associated Dirac operators.

For any $\xi \in \mathfrak{k}^{*}$, the stabilizer $K_{\xi}$ is a connected subgroup of $K$ with same rank. We denote by $\mathfrak{k}_{\xi}$ its Lie algebra. Let $\mathcal{H}_{\mathfrak{k}}$ be the set of conjugacy classes of the reductive algebras $\mathfrak{k}_{\xi}, \xi \in \mathfrak{k}^{*}$. It contains the conjugacy class formed by the Cartan sub-algebras, and it contains also $\mathfrak{k}$ (stabilizer of 0 ).

We denote by $\mathcal{S}_{\mathfrak{k}}$ the set of conjugacy classes of the semi-simple parts $[\mathfrak{h}, \mathfrak{h}]$ of the elements $(\mathfrak{h}) \in \mathcal{H}_{\mathfrak{k}}$. The map $(\mathfrak{h}) \rightarrow([\mathfrak{h}, \mathfrak{h}])$ induces a bijection between $\mathcal{H}_{\mathfrak{k}}$ and $\mathcal{S}_{\mathfrak{k}}$ (see [29]).

We group the coadjoint orbits according to the conjugacy class $(\mathfrak{h}) \in \mathcal{H}_{\mathfrak{k}}$ of the stabilizer, and we consider the Dixmier sheet $\mathfrak{k}_{(\mathfrak{h})}^{*}$ of orbits $K \xi$ with $\mathfrak{k}_{\xi}$ conjugated to $\mathfrak{h}$. The connected Lie subgroup with Lie algebra $\mathfrak{h}$ is denoted $H$, that is if $\mathfrak{h}=\mathfrak{k}_{\xi}$, then $H=K_{\xi}$. We write $\mathfrak{h}=\mathfrak{z} \oplus[\mathfrak{h}, \mathfrak{h}]$, where $\mathfrak{z}$ is the center and $[\mathfrak{h}, \mathfrak{h}]$ is the semi-simple part of $\mathfrak{h}$. Thus $\mathfrak{h}^{*}=\mathfrak{z}^{*} \oplus[\mathfrak{h}, \mathfrak{h}]^{*}$, and $\mathfrak{z}^{*}$ is the set of elements in $\mathfrak{h}^{*}$ vanishing on the semi-simple part of $\mathfrak{h}$. We write $\mathfrak{k}=\mathfrak{h} \oplus[\mathfrak{z}, \mathfrak{k}]$, so we embed $\mathfrak{h}^{*}$ in $\mathfrak{k}^{*}$ as an $H$-invariant subspace, that is we consider an element $\xi \in \mathfrak{h}^{*}$ also as an element of $\mathfrak{k}^{*}$ vanishing on $[\mathfrak{z}, \mathfrak{k}]$. Let $\mathfrak{z}_{0}^{*}$ be the set of $\xi \in \mathfrak{z}^{*}$, such that $\mathfrak{k}_{\xi}=\mathfrak{h}$. We see then that the Dixmier sheet $\mathfrak{k}_{(\mathfrak{h})}^{*}$ is equal to $K \cdot \mathfrak{z}_{0}^{*}$.

\subsection{Admissible coadjoint orbits}

We first define the $\varrho$-orbit. Let $T$ be a Cartan subgroup of $K$. Then $\mathfrak{t}^{*}$ is embedded in $\mathfrak{k}^{*}$ as the subspace of $T$-invariant elements. Choose a system of positive roots $\Delta^{+} \subset \mathfrak{t}^{*}$, and let $\varrho^{K}=\frac{1}{2} \sum_{\alpha>0} \alpha$. The definition of $\varrho^{K}$ requires the choice of a Cartan subgroup $T$ and of a positive root system. However a different choice leads to a conjugate element. Thus we can make the following definition.

Definition 3.1. We denote by $o(\mathfrak{k})$ the coadjoint orbit of $\varrho^{K} \in \mathfrak{k}^{*}$. We call $o(\mathfrak{k})$ the $\varrho$-orbit.

If $K$ is abelian, then $o(\mathfrak{k})$ is $\{0\}$.

The notion of admissible coadjoint orbit is defined in [8] for any real Lie group $G$. When $K$ is a compact connected Lie group, we adopt the following equivalent definition: a coadjoint orbit $\mathcal{O} \subset \mathfrak{k}^{*}$ is admissible if $\mathcal{O}$ carries a $K$-equivariant spin ${ }^{c}$-bundle $\mathcal{S}_{\mathcal{O}}$, such that the associated $K$-equivariant moment map is the injection $\mathcal{O} \hookrightarrow \mathfrak{k}^{*}$ (by equivariance the moment map is unique). If $K \xi$ is an admissible orbit, we also say that the element $\xi$ is admissible. An admissible coadjoint orbit $\mathcal{O}$ is oriented by its symplectic structure, and we denote by $\mathrm{Q}_{K}^{\mathrm{spin}}(\mathcal{O}):=\mathcal{Q}_{K}\left(\mathcal{O}, \mathcal{S}_{\mathcal{O}}\right)$ the corresponding equivariant $\operatorname{spin}^{c}$ index. 
We have $\left\langle\xi,\left[\mathfrak{k}_{\xi}, \mathfrak{k}_{\xi}\right]\right\rangle=0$. The quotient space $\mathfrak{q}=\mathfrak{k} / \mathfrak{k}_{\xi}$ is equipped with the symplectic form $\Omega_{\xi}(\bar{X}, \bar{Y}):=\langle\xi,[X, Y]\rangle$, and with a unique $K_{\xi}$-invariant complex structure $J_{\xi}$ such that $\Omega_{\xi}\left(\cdot, J_{\xi} \cdot\right)$ is a scalar product. We denote by $\mathfrak{q}^{\xi}$ the complex $K_{\xi}$-module $\left(\mathfrak{k} / \mathfrak{k}_{\xi}, J_{\xi}\right)$, and by $\operatorname{Tr}_{\mathfrak{q}^{\xi}}^{\mathbb{C}}: \operatorname{End}\left(\mathfrak{q}^{\xi}\right) \rightarrow \mathbb{C}$ the complex trace.

Any element $X \in \mathfrak{k}_{\xi}$ defines a complex linear $\operatorname{map} \operatorname{ad}(X): \mathfrak{q}^{\xi} \rightarrow \mathfrak{q}^{\xi}$.

Definition 3.2. For any $\xi \in \mathfrak{k}^{*}$, we denote $\varrho(\xi) \in \mathfrak{k}_{\xi}^{*}$ the element defined by

$$
\langle\varrho(\xi), X\rangle=\frac{1}{2 i} \operatorname{Tr}_{\mathfrak{q}}^{\mathbb{C}} \operatorname{ad}(X), \quad X \in \mathfrak{k}_{\xi}
$$

We extend $\varrho(\xi)$ to an element of $\mathfrak{k}^{*}$, that we still denote by $\varrho(\xi)$.

If $i \theta: \mathfrak{k}_{\xi} \rightarrow i \mathbb{R}$ is the differential of a character of $K_{\xi}$, we denote by $\mathbb{C}_{\theta}$ the corresponding 1-dimensional representation of $K_{\xi}$, and by $\left[\mathbb{C}_{\theta}\right]=K \times_{K_{\xi}} \mathbb{C}_{\theta}$ the corresponding line bundle over the coadjoint orbit $K \xi \subset \mathfrak{k}^{*}$. The condition that $K \xi$ is admissible means that there exists a $\operatorname{spin}^{c}$-bundle $\mathcal{S}$ on $K \xi$ such that $\operatorname{det}(\mathcal{S})=\left[\mathbb{C}_{2 \xi}\right](2 i \xi$ needs to be the differential of a character of $K_{\xi}$ ).

LEMMA 3.3. (1) $\left\langle\varrho(\xi),\left[\mathfrak{k}_{\xi}, \mathfrak{k}_{\xi}\right]\right\rangle=0$.

(2) The coadjoint orbit $K \xi$ is admissible if and only if $i(\xi-\varrho(\xi))$ is the differential of a 1-dimensional representation of $K_{\xi}$.

Proof. Consider the character $k \mapsto \operatorname{det}_{\mathfrak{q} \xi}(k)$ of $K_{\xi}$. Its differential is $2 i \varrho(\xi)$. Thus $\left\langle\varrho(\xi),\left[\mathfrak{k}_{\xi}, \mathfrak{k}_{\xi}\right]\right\rangle=0$.

We can equip $K \xi \simeq K / K_{\xi}$ with the $\operatorname{spin}^{c}$-bundle $\mathcal{S}_{\xi}:=K \times_{K_{\xi}} \wedge \mathfrak{q}^{\xi}$ with determinant line bundle $\operatorname{det}\left(\mathcal{S}_{\xi}\right)=\left[\mathbb{C}_{2 \varrho(\xi)}\right]$. Any other $K$-equivariant spin ${ }^{c}$-bundle on $K \xi$ is of the form $\mathcal{S}_{\xi} \otimes\left[\mathbb{C}_{\theta}\right]$, where $i \theta$ is the differential of a character of $K_{\xi}$. Then $\operatorname{det}\left(\mathcal{S}_{\xi} \otimes\left[\mathbb{C}_{\theta}\right]\right)=\left[\mathbb{C}_{2 \xi}\right]$ if and only if $\xi-\varrho(\xi)=\theta$. The lemma then follows.

In particular the orbit $o(\mathfrak{k})$ is admissible. Indeed if $\xi=\varrho^{K}$, then $\xi-\varrho(\xi)=0$.

An admissible coadjoint orbit $\mathcal{P}=K \xi$ is then equipped with the spin ${ }^{c}$-bundle

$$
\mathcal{S}_{\mathcal{P}}^{ \pm}:=K \times_{K_{\xi}}\left(\bigwedge^{ \pm} \mathfrak{q}^{\xi} \otimes \mathbb{C}_{\xi-\varrho(\xi)}\right)
$$

Its $\operatorname{spin}^{c}$ equivariant index is

$$
\mathrm{Q}_{K}^{\operatorname{spin}}(\mathcal{P})=\operatorname{Ind}_{K_{\xi}}^{K}\left(\bigwedge \mathfrak{q}^{\xi} \otimes \mathbb{C}_{\xi-\varrho(\xi)}\right)
$$

See [28].

The following proposition is well known (see [29]). 
Proposition 3.4. - The map $\mathcal{O} \mapsto \pi_{\mathcal{O}}:=\mathrm{Q}_{K}^{\text {spin }}(\mathcal{O})$ defines a bijection between the set of regular admissible orbits and $\widehat{K}$.

- $\mathrm{Q}_{K}^{\mathrm{spin}}(o(\mathfrak{k}))$ is the trivial representation of $K$.

We now describe the representation $\mathrm{Q}_{K}^{\text {spin }}(\mathcal{P})$ attached to any admissible orbit $\mathcal{P}$ in terms of regular admissible orbits.

Definition 3.5. With any coadjoint orbit $\mathcal{P} \subset \mathfrak{k}^{*}$, we associate the coadjoint orbit $s(\mathcal{P}) \subset \mathfrak{k}^{*}$ which is defined as follows: if $\mathcal{P}=K \mu$, take $s(\mathcal{P})=K \xi$ with $\xi \in \mu+o\left(\mathfrak{k}_{\mu}\right)$. We call $s(\mathcal{P})$ the shift of the orbit $\mathcal{P}$.

If $\mathcal{P}$ is regular, $s(\mathcal{P})=\mathcal{P}$. If $\mathcal{P}=\{0\}$, then $s(\mathcal{P})=o(\mathfrak{k})$.

The following proposition summarises the results concerning the quantization of admissible orbits.

Proposition 3.6. ([29]) Let $\mathcal{P}$ be an admissible orbit.

- $\mathcal{P}^{*}:=-\mathcal{P}$ is also admissible and $\mathrm{Q}_{K}^{\text {spin }}\left(\mathcal{P}^{*}\right)=\mathrm{Q}_{K}^{\text {spin }}(\mathcal{P})^{*}$.

- If $s(\mathcal{P})$ is regular, then $s(\mathcal{P})$ is also admissible.

- Conversely, if $\mathcal{O}$ is regular and admissible, and $\mathcal{P}$ is such that $s(\mathcal{P})=\mathcal{O}$, then $\mathcal{P}$ is admissible.

- The following hold:

- If $s(\mathcal{P})$ is not regular, then $\mathrm{Q}_{K}^{\mathrm{spin}}(\mathcal{P})=0$.

- If $s(\mathcal{P})$ is regular, then $\mathrm{Q}_{K}^{\text {spin }}(\mathcal{P})=\mathrm{Q}_{K}^{\text {spin }}(s(\mathcal{P}))=\pi_{s(\mathcal{P})}$.

It is important to understand what are the admissible orbits $\mathcal{P}$ such that $s(\mathcal{P})$ is equal to a fixed regular admissible orbit $\mathcal{O}$.

Definition 3.7. - For a conjugacy class $(\mathfrak{h}) \in \mathcal{H}_{\mathfrak{k}}$, we denote by $\mathcal{A}((\mathfrak{h}))$ the set of admissible orbits belonging to the Dixmier sheet $\mathfrak{k}_{(\mathfrak{h})}^{*}$.

- If $\mathcal{P}, \mathcal{O} \subset \mathfrak{k}^{*}$ are $K$-orbits, $\mathcal{P}$ is called an (h)-ancestor of $\mathcal{O}$ if $\mathcal{P} \subset \mathfrak{k}_{(\mathfrak{h})}^{*}$ and $s(\mathcal{P})=\mathcal{O}$.

We make the choice of a connected Lie subgroup $H$ with Lie algebra $\mathfrak{h}$ and write $\mathfrak{h}=\mathfrak{z} \oplus[\mathfrak{h}, \mathfrak{h}]$. We denote by $\mathfrak{z}_{0}^{*}$ the set of elements $\xi \in \mathfrak{z}^{*}$ such that $K_{\xi}=H$. The orbit $o(\mathfrak{h})$ (the $\varrho$-orbit for $H$ ) is contained in $[\mathfrak{h}, \mathfrak{h}]^{*}$.

An orbit $\mathcal{P}$ is an $(\mathfrak{h})$-ancestor of an orbit $\mathcal{O}$, if and only if there exists $\mu \in \mathfrak{z}_{0}^{*}$ such that $\mathcal{P}=K \mu$ and $\mathcal{O}=K \lambda$ for $\lambda \in \mu+o(\mathfrak{h})$. The following fact that is proved in [29, Theorem 4.4] will be needed in the next sections.

LEMma 3.8. Let $\mathcal{P}$ be a $(\mathfrak{h})$-ancestor of a regular admissible orbit $\mathcal{O}$. Take $\mu \in \mathcal{P} \cap \mathfrak{z}_{0}^{*}$ and $\lambda \in \mu+o(\mathfrak{h})$. Then the form $\lambda-\varrho(\lambda)$ is equal to $\mu-\varrho(\mu) \in \mathfrak{z}^{*}$ and corresponds $\left({ }^{3}\right)$ to the differential of a character of $H$.

$\left({ }^{3}\right)$ Modulo the " $i$ " factor. 


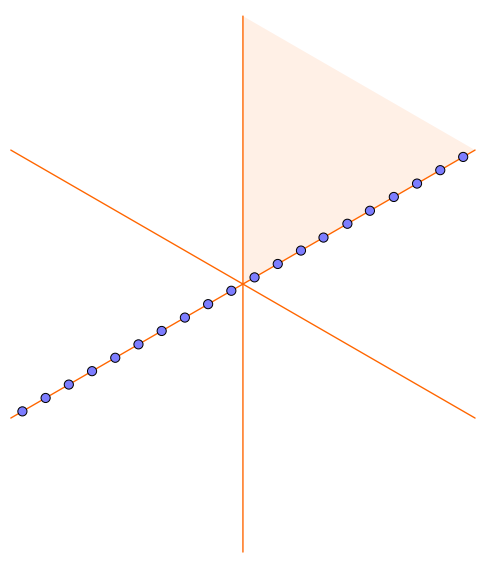

Figure 4. $H$-admissible orbits.

Given a regular admissible orbit $\mathcal{O}$, there might be several $(\mathfrak{h})$-ancestors to $\mathcal{O}$. There might also be several classes of conjugacy $(\mathfrak{h})$ such that $\mathcal{O}$ admits an $(\mathfrak{h})$-ancestor $\mathcal{P}$. For example, let $\mathcal{O}=o(\mathfrak{k})$. Then, for any $\mathfrak{h} \in \mathcal{H}_{\mathfrak{k}}$, the orbit $K\left(\varrho^{K}-\varrho^{H}\right)$ is an $(\mathfrak{h})$-ancestor to $\mathcal{O}$. Here we have chosen a Cartan subgroup $T$ contained in $H, H=K_{\xi}$ and a positive root system such that $\xi$ is dominant to define $\varrho^{K}$ and $\varrho^{H}$.

Example 3.9. Consider the group $K=\mathrm{SU}(3)$ and let $(\mathfrak{h})$ be the centralizer class of a subregular element $f \in \mathfrak{k}^{*}$ with centralizer $H=S(U(2) \times U(1))$.

We consider the Cartan subalgebra of diagonal matrices and choose a Weyl chamber. Let $\omega_{1}$ and $\omega_{2}$ be the two fundamental weights. Let $\sigma_{1}$ and $\sigma_{2}$ be the half-lines $\mathbb{R}_{>0} \omega_{1}$ and $\mathbb{R}_{>0} \omega_{2}$. The set $\mathcal{A}((\mathfrak{h}))$ is equal to the collection of orbits $K \cdot\left(\frac{1}{2}(1+2 n) \omega_{1}\right), n \in \mathbb{Z}$ (see Figure 4).

As $-\omega_{1}$ is conjugated to $\omega_{2}$, we see that the set $\mathcal{A}((\mathfrak{h}))$ is equal to the collection of orbits $K \cdot\left(\frac{1}{2}(1+2 n) \omega_{i}\right), n \in \mathbb{Z}_{\geqslant 0}, i=1,2$. Here we have chosen the representatives in the chosen closed Weyl chamber.

One has $s\left(K \cdot\left(\frac{1}{2}(1+2 n) \omega_{i}\right)\right)=K\left(\varrho^{K}+(n-1) \omega_{i}\right)$. Thus the shifted orbit is a regular orbit if and only if $n>0$. For $n=1$, both admissible orbits $K \cdot \frac{3}{2} \omega_{1}$ and $K \cdot\left(-\frac{3}{2} \omega_{1}\right)=K \cdot \frac{3}{2} \omega_{2}$ are $(\mathfrak{h})$-ancestors to the orbit $K \varrho^{K}=o(\mathfrak{k})$.

Both admissible orbits $\mathcal{P}_{1}=K \cdot \frac{1}{2} \omega_{1}$ and $\mathcal{P}_{2}=K \cdot \frac{1}{2} \omega_{2}$ are such that $\mathrm{Q}_{K}^{\text {spin }}\left(\mathcal{P}_{i}\right)=0$.

In Figure 5, we draw the link between $H$-admissible orbits and their respective shifts. 


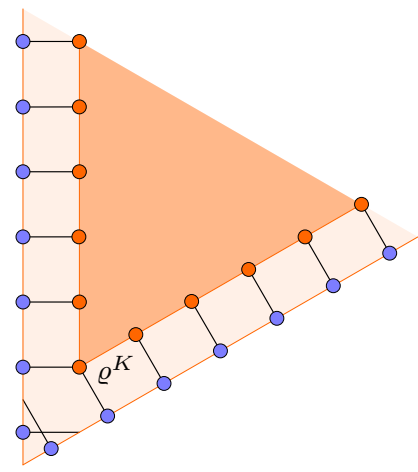

Figure 5. $H$-admissible orbits and their shifts.

\subsection{Magical inequality}

We often will use complex structures and normalized traces on real vector spaces defined by the following procedure.

Definition 3.10. Let $N$ be a real vector space and $b: N \rightarrow N$ a linear transformation, such that $-b^{2}$ is diagonalizable with non-negative eigenvalues.

- We define the diagonalizable transformation $|b|=\sqrt{-b^{2}}$ of $N$.

- We define the complex structure $J_{b}=b|b|^{-1}$ on $N / \operatorname{ker}(b)$.

- We denote by $\mathbf{n T r}_{N}|b|=\frac{1}{2} \operatorname{Tr}_{N}^{\mathbb{R}}|b|$, that is half of the trace of the action of $|b|$ in the real vector space $N$. We call $\mathbf{n} \operatorname{Tr}_{N}|b|$ the normalized trace of $b$.

If $N$ has a Hermitian structure invariant by $b, \frac{1}{2} \operatorname{Tr}_{N}^{\mathbb{R}}|b|$ is equal to the complex trace of $|b|$ considered as a Hermitian endomorphism of $N$. The interest of our notation is that we do not need complex structures to define $\mathbf{n} \mathbf{T r}_{N}|b|$.

If $N$ is a Euclidean space and $b$ a skew-symmetric transformation of $N$, then $-b^{2}$ is diagonalizable with non-negative eigenvalues. By definition of $J_{b}$, the transformation $b$ of $N$ determines a complex diagonalizable transformation of $N / \operatorname{ker}(b)$, and the list of its complex eigenvalues is $\left[i a_{1}, \ldots, i a_{\ell}\right]$ where the $a_{k}$ are strictly positive real numbers. We have $\mathbf{n T r}_{N}|b|=\sum_{k=1}^{\ell} a_{k} \geqslant 0$.

Recall our identification $\mathfrak{k}=\mathfrak{k}^{*}$ with the help of a scalar product. When $\beta \in \mathfrak{k}^{*}$, denote by $b$ the corresponding element of $\mathfrak{k}$. We have defined a complex structure $J_{\beta}$ on $\mathfrak{k} / \mathfrak{k}_{\beta}$. On the other hand, $b$ defines an invertible transformation of $\mathfrak{k} / \mathfrak{k}_{\beta}$. It can be checked that $J_{\beta}=J_{b}$. If we choose a Cartan subalgebra containing $b$, then $\mathbf{n T r}_{\mathfrak{k}}|b|=\sum_{\alpha>0}|\langle\alpha, b\rangle|$.

For further use, we include a lemma. Let us consider $\mathfrak{k}_{\mathbb{C}}$, the complexified space of $\mathfrak{k}$. Consider the complex space $\bigwedge \mathfrak{k}_{\mathbb{C}}$. 
Lemma 3.11. Let $b \in \mathfrak{k}$. Let $x \in \mathbb{R}$ be an eigenvalue for the action of $b / i$ in $\bigwedge \mathfrak{k}_{\mathbb{C}}$. Then $x \geqslant-\mathbf{n} \operatorname{Tr}_{\mathfrak{k}}|b|$.

Proof. Indeed, consider a Cartan subalgebra $\mathfrak{t}$ containing $b$, the system of roots $\Delta$ and an order such that $\langle\alpha, b\rangle \geqslant 0$ for all $\alpha>0$. An eigenvalue $x$ on $\Lambda \mathfrak{k}_{\mathbb{C}}$ is thus of the form $\sum_{\alpha \in I \subset \Delta}\langle\alpha, b\rangle$. Thus we see that the lowest eigenvalue is $-\sum_{\alpha>0}\langle\alpha, b\rangle=-\mathbf{n} \mathbf{T r}_{\mathfrak{k}}|b|$.

Assume now that $\mathcal{N} \rightarrow M$ is a real vector bundle equipped with an action of a compact Lie group $K$. For any $b \in \mathfrak{k}$, and any $m \in M$ such that $b_{M}(m)=0$, we may consider the linear action $\left.\mathcal{L}(b)\right|_{m}$ which is induced by $b$ on the fibers $\left.\mathcal{N}\right|_{m}$. It is easy to check that $\left(\left.\mathcal{L}(b)\right|_{m}\right)^{2}$ is diagonalizable with eigenvalues which are negative or equal to zero. We denote by $|\mathcal{L}(b)|_{m} \mid=\sqrt{-\left(\left.\mathcal{L}(b)\right|_{m}\right)^{2}}$.

Definition 3.12. We denote by $\mathbf{n T r}_{\left.\mathcal{N}\right|_{m}}|b|:=\frac{1}{2} \operatorname{Tr}_{\mathcal{N}_{m}}^{\mathbb{R}}|\mathcal{L}(b)|_{m} \mid$ that is half of the trace of the real endomorphism $|\mathcal{L}(b)|_{m} \mid$ on $\left.\mathcal{N}\right|_{m}$. We call $\mathbf{n T r}_{\left.\mathcal{N}\right|_{m}}|b|$ the normalized trace of the action of $b$ on $\left.\mathcal{N}\right|_{m}$.

For any $b \in \mathfrak{k}$ and $\mu \in \mathfrak{k}^{*}$ fixed by $b$, we may consider the action $\operatorname{ad}(b): \mathfrak{k}_{\mu} \rightarrow \mathfrak{k}_{\mu}$ and the corresponding normalized trace $\mathbf{n} \mathbf{T r}_{\mathfrak{k}_{\mu}}|\operatorname{ad}(b)|$ denoted simply by $\mathbf{n} \mathbf{T r}_{\mathfrak{k}_{\mu}}|b|$.

Definition 3.13. A regular element $\lambda \in \mathfrak{k}^{*}$ determines a closed positive Weyl chamber $C_{\lambda} \subset \mathfrak{k}_{\lambda}^{*}$. We say that $\lambda$ is very regular if $\lambda \in \varrho(\lambda)+C_{\lambda}$.

Notice that regular admissible elements are very regular.

The following "magical inequality", that is proved in [29], will be a crucial tool in $\oint 4.5$.

Proposition 3.14. (Magical inequality) Let $b \in \mathfrak{k}$ and denote by $\beta$ the corresponding element in $\mathfrak{k}^{*}$. Let $\lambda$ and $\mu$ be elements of $\mathfrak{k}^{*}$ fixed by $b$. Assume that $\lambda$ is very regular and that $\mu-\lambda=\beta$. Then

$$
\|\beta\|^{2} \geqslant \frac{1}{2} \mathbf{n} \operatorname{Tr}_{\mathfrak{k}_{\mu}}|b| .
$$

The equality holds if and only if one of the following equivalent statements holds:

(a) $\lambda \in \mu+o\left(\mathfrak{k}_{\mu}\right)$

(b) $\mu \in C_{\lambda}$ and $\lambda-\varrho(\lambda)=\mu-\varrho(\mu)$.

\subsection{Slices and induced $\operatorname{spin}^{c}$-bundles}

We suppose here that $M$ is a $K$-manifold and that $\Phi: M \rightarrow \mathfrak{k}^{*}$ is a $K$-equivariant map. If $\mathcal{O}$ is a coadjoint orbit, a neighborhood of $\Phi^{-1}(\mathcal{O})$ in $M$ can be identified with an induced manifold, and the restriction of $\operatorname{spin}^{c}$-bundles to a neighborhood of $\Phi^{-1}(\mathcal{O})$ can be identified to an induced bundle. To this aim, let us recall the notion of slice [21]. 
Definition 3.15. Let $M$ be a $K$-manifold and $m \in M$ with stabilizer subgroup $K_{m}$. A submanifold $Y \subset M$ containing $m$ is a slice at $m$ if $Y$ is $K_{m}$-invariant, $K Y$ is a neighborhood of $m$, and the map

$$
\begin{array}{r}
K \times_{K_{m}} Y \longrightarrow M, \\
{[k, y] \longmapsto k y,}
\end{array}
$$

is an isomorphism onto $K Y$.

Consider the coadjoint action of $K$ on $\mathfrak{k}^{*}$. Let us fix $\mu \in \mathfrak{k}^{*}$ and $H:=K_{\mu}$. Let $C$ be the connected component of the open subset

$$
\mathfrak{h}_{0}^{*}:=\left\{\xi \in \mathfrak{h}^{*}: K_{\xi} \subset H\right\}
$$

containing $\mu$. The map $K \times{ }_{H} C \rightarrow K C$ is a diffeomorphism. Thus $C$ is a slice at $\mu$ for the coadjoint action.

The following construction was used as a fundamental tool in the symplectic setting [14].

Proposition 3.16. Let $\Phi: M \rightarrow \mathfrak{k}^{*}$ be a $K$-invariant map. Let $\mu \in \mathfrak{k}^{*}$, and let $C$ be the slice at $\mu$ defined previously.

- $\mathcal{Y}_{C}=\Phi^{-1}(C)$ is a $K_{\mu}$-invariant submanifold of $M$ (perhaps empty).

- $K C$ is an open neighborhood of $\Phi^{-1}(K \mu)$ diffeomorphic to $K \times_{K_{\mu}} \mathcal{Y}_{C}$.

The manifold $\mathcal{Y}_{C}$, when is not empty, is called the slice (of $M$ ) at $\mu \in \mathfrak{k}^{*}$. Note that $\mathcal{Y}_{C}$ can be disconnected.

Proof. Let us consider the $H=K_{\mu}$ invariant orthogonal decompositions $\mathfrak{k}=\mathfrak{h} \oplus \mathfrak{q}$ and $\mathfrak{k}^{*}=\mathfrak{h}^{*} \oplus \mathfrak{q}^{*}$ : we denote $\xi \rightarrow[\xi]_{\mathfrak{q}^{*}}$ the corresponding projection to $\mathfrak{q}^{*}$.

A point $\xi$ is in $\mathfrak{h}_{0}^{*}$ (see (3.14)) if and only if the map $X \mapsto \xi \circ \operatorname{ad}(X)$ is an isomorphism from $\mathfrak{q}$ to $\mathfrak{q}^{*}$. Thus, for any $y \in \mathcal{Y}_{C}$, the linear map $\Pi_{y}:=[\cdot]_{\mathfrak{q}^{*}} \circ \mathrm{T}_{y} \Phi: \mathrm{T}_{y} M \rightarrow \mathfrak{q}^{*}$ is onto. Indeed, the tangent space to $K y$ projects onto the tangent space to $K \Phi(y)$, which contains $[\mathfrak{q}, \Phi(y)]=\mathfrak{q}^{*}$. Thus we obtain that $\mathcal{Y}_{C}$ is a submanifold with tangent space $\operatorname{ker}\left(\Pi_{y}\right)$ and furthermore $\mathrm{T}_{y} M=\mathrm{T}_{y} \mathcal{Y}_{C} \oplus \mathfrak{q} \cdot y$.

The rest of the assertions follow from the fact that $C$ is a slice at $\mu$ for the coadjoint action.

Suppose now that $M$ is oriented and carries a $K$-equivariant $\operatorname{spin}^{c}$-bundle $\mathcal{S}$. Let us explain how this data induces a $\operatorname{spin}^{c}$-bundle on the slice $\mathcal{Y}_{C}$.

Any element $\xi \in \mathfrak{h}_{0}^{*}$ determines a complex structure $\left(^{4}\right) J_{\xi}=\operatorname{ad}(\xi)|\operatorname{ad}(\xi)|^{-1}$ on $\mathfrak{q}:=\mathfrak{k} / \mathfrak{h}$ which depends only of the connected component $C$ of $\mathfrak{h}_{0}^{*}$ containing $\xi$ : thus we denote

( $\left.{ }^{4}\right)$ The element $\xi$ is viewed as an element of $\mathfrak{h}$ through the identification $\mathfrak{k} \simeq \mathfrak{k}^{*}$. 
by $J_{C}$ the corresponding complex structure on $\mathfrak{q}:=\mathfrak{k} / \mathfrak{h}$. We denote by $\mathfrak{q}^{C}$ the complex $H$-module $\left(\mathfrak{q}, J_{C}\right)$, and $\varrho_{C}$ the element of $\mathfrak{z}^{*}$ defined by the relation

$$
\left\langle\varrho_{C}, X\right\rangle=\frac{1}{2 i} \operatorname{Tr}_{\mathfrak{q}^{C}}^{\mathbb{C}} \operatorname{ad}(X), \quad X \in \mathfrak{h} .
$$

Consider the $H$-manifold $\mathcal{Y}_{C}$ and the open subset $K \mathcal{Y}_{C} \simeq K \times_{H} \mathcal{Y}_{C}$ of $M$. At the level of tangent spaces we have $\left.\mathrm{T} M\right|_{\mathcal{Y}_{C}}=[\mathfrak{q}] \oplus \mathrm{T} \mathcal{Y}_{C}$. We orient the manifold $Y$ through the relation $o(M)=o\left(J_{C}\right) o\left(\mathcal{Y}_{C}\right)$. The restriction of the $\operatorname{spin}^{c}$-bundle $\mathcal{S}$ to the submanifold $\mathcal{Y}_{C}$ permits to define the unique $\operatorname{spin}^{c}$-bundle $\mathcal{S}_{\mathcal{Y}_{C}}$ on $\mathcal{Y}_{C}$ such that

$$
\left.\mathcal{S}\right|_{\mathcal{Y}_{C}}=\bigwedge \mathfrak{q}^{C} \otimes \mathcal{S}_{\mathcal{Y}_{C}} .
$$

This gives a bijection between the $K$-equivariant $\operatorname{spin}^{c}$-bundles on $K \times_{H} \mathcal{Y}_{C}$ and the $H$ equivariant $\operatorname{spin}^{c}$-bundles on $\mathcal{Y}_{C}$. If the relation (3.16) holds, we say that $\mathcal{S}_{\mathcal{Y}_{C}}$ is the $\operatorname{spin}^{c}$-bundle induced by $\mathcal{S}$. Notice that at the level of determinant line bundles we have

$$
\left.\operatorname{det}(\mathcal{S})\right|_{\mathcal{Y}_{C}}=\operatorname{det}\left(\mathcal{S}_{\mathcal{Y}_{C}}\right) \otimes \mathbb{C}_{2 \varrho_{C}} .
$$

Let us consider the particular situation where the slice $\mathcal{Y}_{C}$ is a compact submanifold of $M$. It is the case when $M=K \times_{H} \mathcal{Y}_{C}$, and in this setting we have a simple formula that relate the $\operatorname{spin}^{c}$-indices on $M$ and on the slice $\mathcal{Y}_{C}$ :

$$
\mathcal{Q}_{K}(M, \mathcal{S})=\operatorname{Ind}_{H}^{K}\left(\bigwedge \mathfrak{q}^{C} \otimes \mathcal{Q}_{H}\left(\mathcal{Y}_{C}, \mathcal{S}_{\mathcal{Y}_{C}}\right)\right)
$$

See $[28]$.

\section{Computing the multiplicities}

\subsection{Transversally elliptic operators}

In this subsection, we recall the basic definitions from the theory of transversally elliptic symbols (or operators) defined by Atiyah and Singer in [1]. We refer to [5] and [27] for more details.

Let $M$ be a compact $K$-manifold with cotangent bundle $\mathrm{T}^{*} M$. Let $p: \mathrm{T}^{*} M \rightarrow M$ be the projection. If $\mathcal{E}$ is a vector bundle on $M$, we may denote still by $\mathcal{E}$ the vector bundle $p^{*} \mathcal{E}$ on the cotangent bundle $\mathrm{T}^{*} M$. If $\mathcal{E}^{+}$and $\mathcal{E}^{-}$are $K$-equivariant complex vector bundles over $M$, a $K$-equivariant morphism $\sigma \in \Gamma\left(\mathrm{T}^{*} M, \operatorname{hom}\left(\mathcal{E}^{+}, \mathcal{E}^{-}\right)\right)$is called a symbol on $M$. For $x \in M$, and $\nu \in T_{x}^{*} M$, thus $\sigma(x, \nu): \mathcal{E}_{x}^{+} \rightarrow \mathcal{E}_{x}^{-}$is a linear map from $\mathcal{E}_{x}^{+}$to $\mathcal{E}_{x}^{-}$. The subset of all $(x, \nu) \in \mathrm{T}^{*} M$, where the map $\sigma(x, \nu)$ is not invertible, is called the characteristic set of $\sigma$, and is denoted by Char $(\sigma)$. A symbol is elliptic if its 
characteristic set is compact. An elliptic symbol $\sigma$ on $M$ defines an element $[\sigma]$ in the equivariant $\mathbf{K}$-theory of $\mathrm{T}^{*} M$ with compact support, which is denoted by $\mathbf{K}_{K}^{0}\left(\mathrm{~T}^{*} M\right)$. The index of $\sigma$ is a virtual finite-dimensional representation of $K$, that we denote by Index $\operatorname{Me}_{K}^{M}(\sigma) \in R(K)$.

Recall the notion of transversally elliptic symbol. Let $\mathrm{T}_{K}^{*} M$ be the following $K$ invariant closed subset of $\mathrm{T}^{*} M$

$$
\mathrm{T}_{K}^{*} M=\left\{(x, \nu) \in \mathrm{T}^{*} M:\langle\nu, X \cdot x\rangle=0 \text { for all } X \in \mathfrak{k}\right\} .
$$

Its fiber over a point $x \in M$ is formed by all the cotangent vectors $\nu \in T_{x}^{*} M$ which vanish on the tangent space to the orbit of $x$ under $K$, in the point $x$. A symbol $\sigma$ is $K$-transversally elliptic if the restriction of $\sigma$ to $\mathrm{T}_{K}^{*} M$ is invertible outside a compact subset of $\mathrm{T}_{K}^{*} M$ (i.e. $\operatorname{Char}(\sigma) \cap \mathrm{T}_{K}^{*} M$ is compact).

A $K$-transversally elliptic symbol $\sigma$ defines an element of $\mathbf{K}_{K}^{0}\left(\mathrm{~T}_{K}^{*} M\right)$, and the index of $\sigma$ defines an element $\operatorname{Index}_{K}^{M}(\sigma)$ of $\widehat{R}(K)$ defined in [1].

We will use the following obvious remark. Let $\sigma \in \Gamma\left(\mathrm{T}^{*} M, \operatorname{hom}\left(\mathcal{E}^{+}, \mathcal{E}^{-}\right)\right)$be a transversally elliptic symbol on $M$.

LEMMA 4.1. Assume that an element $b \in K$ acts trivially on $M$, and that $\mathcal{E}^{ \pm}$are $K$-equivariant vector bundles on $M$ such that the subbundles $\left[\mathcal{E}^{ \pm}\right]^{b}$ fixed by $b$ are equal to $\{0\}$. Then $\left[\operatorname{Index}{ }_{K}^{M}(\sigma)\right]^{K}=0$

Proof. The space $\left[\operatorname{Index}_{K}^{M}(\sigma)\right]^{K}$ is constructed as the (virtual) subspace of invariant $C^{\infty}$-sections of the bundle $\mathcal{E}^{ \pm}$which are solutions of a $K$-invariant pseudo-differential operator on $M$ with symbol $\sigma$. But, as the action of $b$ is trivial on the basis, and $\left[\mathcal{E}^{ \pm}\right]^{b}=\{0\}$, the space of $b$-invariant $C^{\infty}$-sections of the bundle $\mathcal{E}^{ \pm}$is reduced to zero.

Any elliptic symbol is $K$-transversally elliptic, hence we have a restriction map $\mathbf{K}_{K}^{0}\left(\mathrm{~T}^{*} M\right) \rightarrow \mathbf{K}_{K}^{0}\left(\mathrm{~T}_{K}^{*} M\right)$, and a commutative diagram

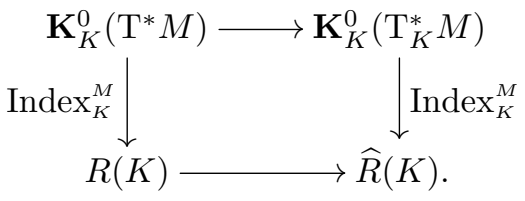

Using the excision property, one can easily show that the index map

$$
\operatorname{Index}_{K}: \mathbf{K}_{K}^{0}\left(\mathrm{~T}_{K}^{*} \mathcal{U}\right) \longrightarrow \widehat{R}(K)
$$

is still defined when $\mathcal{U}$ is a $K$-invariant relatively compact open subset of a $K$-manifold (see $[26, \S 3.1])$.

In the rest of this article, $M$ will be a Riemannian manifold, and we denote by $\nu \in \mathrm{T}^{*} M \rightarrow \tilde{\nu} \in \mathrm{T} M$ the corresponding identification. 


\subsection{The Witten deformation}

In this section $M$ is an oriented $K$-manifold of even dimension (not necessarily compact). Let $\Phi: M \rightarrow \mathfrak{k}^{*}$ be a $K$-equivariant map. Let $\varkappa_{\Phi}$ be the Kirwan vector field associated with $\Phi$ (see (2.10)). We denote by $Z_{\Phi}$ the set of zeros of $\varkappa_{\Phi}$ : clearly $Z_{\Phi}$ contains the set of fixed points of the action of $K$ on $M$ as well as $\left.\Phi^{-1}(0)\right)$.

Definition 4.2. Let $\sigma(M, \mathcal{S})(m, \nu)=\mathbf{c}_{\mathcal{S}_{m}}(\tilde{\nu}): \mathcal{S}_{m}^{+} \rightarrow \mathcal{S}_{m}^{-}$be the symbol of the Dirac operator attached to the $\operatorname{spin}^{c}$-bundle $\mathcal{S}$, and let $\Phi: M \rightarrow \mathfrak{k}^{*}$ be an equivariant map. The symbol $\sigma(M, \mathcal{S}, \Phi)$ pushed by the vector field $\varkappa_{\Phi}$ is the symbol defined by

$$
\sigma(M, \mathcal{S}, \Phi)(m, \nu)=\mathbf{c}_{\mathcal{S}_{m}}\left(\tilde{\nu}-\varkappa_{\Phi}(m)\right): \mathcal{S}_{m}^{+} \longrightarrow \mathcal{S}_{m}^{-}
$$

for any $(m, \nu) \in \mathrm{T} M$.

Similarly, if $\mathcal{W} \rightarrow M$ is a $K$-equivariant vector bundle, we define

$$
\sigma(M, \mathcal{S} \otimes \mathcal{W}, \Phi)(m, \nu)=\sigma(M, \mathcal{S}, \Phi)(m, \nu) \otimes \operatorname{Id}_{\mathcal{W}_{m}} .
$$

Note that $\sigma(M, \mathcal{S}, \Phi)(m, \nu)$ is invertible except if $\tilde{\nu}=\varkappa_{\Phi}(m)$. If furthermore $(m, \nu)$ belongs to the subset $\mathrm{T}_{K}^{*} M$ of cotangent vectors orthogonal to the $K$-orbits, then $\nu=0$ and $\varkappa_{\Phi}(m)=0$. Indeed $\varkappa_{\Phi}(m)$ is tangent to $K \cdot m$, while $\tilde{\nu}$ is orthogonal. So we note that $(m, \nu) \in \operatorname{Char}\left(\sigma\left(M, \mathcal{S}, \Phi_{\mathcal{S}}\right)\right) \cap \mathrm{T}_{K}^{*} M$ if and only if $\nu=0$ and $\varkappa_{\Phi}(m)=0$.

For any $K$-invariant open subset $\mathcal{U} \subset M$ such that $\mathcal{U} \cap Z_{\Phi}$ is compact in $M$, we see that the restriction $\left.\sigma(M, \mathcal{S}, \Phi)\right|_{\mathcal{U}}$ is a transversally elliptic symbol on $\mathcal{U}$, and so its equivariant index is a well-defined element in $\widehat{R}(K)$.

Thus, we can define the following localized equivariant indices.

Definition 4.3. - A closed invariant subset $Z \subset Z_{\Phi}$ is called a component if it is a union of connected components of $Z_{\Phi}$.

- If $Z \subset Z_{\Phi}$ is a compact component, and $\mathcal{W}$ is a $K$-equivariant vector bundle over $M$, we denote by

$$
\mathcal{Q}_{K}(M, \mathcal{S} \otimes \mathcal{W}, Z, \Phi) \in \widehat{R}(K)
$$

the equivariant index of $\left.\sigma(M, \mathcal{S} \otimes \mathcal{W}, \Phi)\right|_{\mathcal{U}}$, where $\mathcal{U}$ is an invariant neighborhood of $Z$ so that $\mathcal{U} \cap Z_{\Phi}=Z$.

- If we make the Witten deformation with the map $\Phi=\Phi_{\mathcal{S}}$, then we denote the term $\mathcal{Q}_{K}\left(M, \mathcal{S} \otimes \mathcal{W}, Z, \Phi_{\mathcal{S}}\right)$ simply by $\mathcal{Q}_{K}(M, \mathcal{S} \otimes \mathcal{W}, Z)$.

By definition, $Z=\varnothing$ is a component and $\mathcal{Q}_{K}(M, \mathcal{S} \otimes \mathcal{W}, \varnothing, \Phi)=0$.

When $M$ is compact, it is clear that the classes of the symbols $\sigma(M, \mathcal{S}, \Phi)$ and $\sigma(M, \mathcal{S})$ are equal in $\mathbf{K}_{K}^{0}\left(\mathrm{~T}_{K}^{*} M\right)$, thus we get the first form of the localization theorem. 
THEOREM 4.4. Assume that $M$ is compact. If $Z_{\Phi}=Z_{1} \amalg \ldots \amalg Z_{p}$ is a decomposition into disjoint (compact) components, we have the following equality in $\widehat{R}(K)$ :

$$
\mathcal{Q}_{K}(M, \mathcal{S})=\sum_{i=1}^{p} \mathcal{Q}_{K}\left(M, \mathcal{S}, Z_{i}, \Phi\right)
$$

Remark 4.5. Write $\Phi_{\mathcal{S}}\left(Z_{\mathcal{S}}\right)=\coprod_{j} \mathcal{O}_{j}$ as a disjoint union of a finite set of coadjoint orbits. Then we obtain the decomposition

$$
\mathcal{Q}_{K}(M, \mathcal{S})=\sum_{j} \mathcal{Q}_{\mathcal{O}_{j}}
$$

with $\mathcal{Q}_{\mathcal{O}}=\mathcal{Q}_{K}\left(M, \mathcal{S}, \Phi_{\mathcal{S}}^{-1}(\mathcal{O}) \cap Z_{\mathcal{S}}\right)$. As in [26], this decomposition is the main tool of our study. However, in this work, we will need to introduce a further refinement of this decomposition.

Example 4.6. We return to our basic example (Example 2.14). Let $p_{+}=[1,0]$ and $p_{-}=[0,1]$ be the fixed points of the $T$-action on $M=\mathbb{P}^{1}(\mathbb{C})$. The determinant line bundle of $\mathcal{S}(n)$ is $\mathbb{L}_{n}=\left[\mathbb{C}_{-1}\right] \otimes \mathcal{L}^{\otimes 2 n+2}$, where $\left[\mathbb{C}_{-1}\right]$ is the trivial line bundle equipped with the representation $t^{-1}$ on $\mathbb{C}$. We choose the moment map $\Phi_{n}$ associated with a connection on the determinant bundle (see more details in $\S 6$ ):

$$
\Phi_{n}\left(\left[z_{1}, z_{2}\right]\right)=(n+1) \frac{\left|z_{1}\right|^{2}}{\left|z_{1}\right|^{2}+\left|z_{2}\right|^{2}}-\frac{1}{2} .
$$

Then, for $n \geqslant 0, Z_{\mathcal{S}}=\left\{p_{+}\right\} \cup\left\{p_{-}\right\} \cup \Phi_{n}^{-1}(0)$, thus $\Phi_{n}\left(Z_{\mathcal{S}}\right)=\left\{-\frac{1}{2}\right\} \cup\{0\} \cup\left\{n+\frac{1}{2}\right\}$. Remark that $Z_{\mathcal{S}}$ is smooth: it has three connected components, the two fixed points, and $\Phi_{n}^{-1}(0)$ a circle with free action of $T$. Then we obtain the associated decomposition

$$
\mathcal{Q}_{T}(M, \mathcal{S}(n))=Q_{-1 / 2}+Q_{0}+Q_{1 / 2},
$$

with

$$
Q_{-1 / 2}=-\sum_{k=-1}^{-\infty} t^{k}, \quad Q_{0}=\sum_{k=-\infty}^{-\infty} t^{k} \quad \text { and } \quad Q_{1 / 2}=-\sum_{k=n+1}^{\infty} t^{k} .
$$

Example 4.7. Take the product $N=\mathbb{P}^{1}(\mathbb{C}) \times \mathbb{P}^{1}(\mathbb{C})$, with spin bundle $\mathcal{S}=\mathcal{S}(0) \otimes \mathcal{S}(0)$, moment map $\Phi_{0}$ and we consider the diagonal action of $T$ with moment map

$$
\Phi\left(m_{1}, m_{2}\right)=\Phi_{0}\left(m_{1}\right)+\Phi_{0}\left(m_{2}\right) .
$$

As $\mathcal{Q}_{T}\left(\mathbb{P}^{1}(\mathbb{C}), \mathcal{S}(0)\right)$ is the trivial representation of $T, \mathcal{Q}_{T}(N, \mathcal{S})$ is still the trivial representation of $T$. 

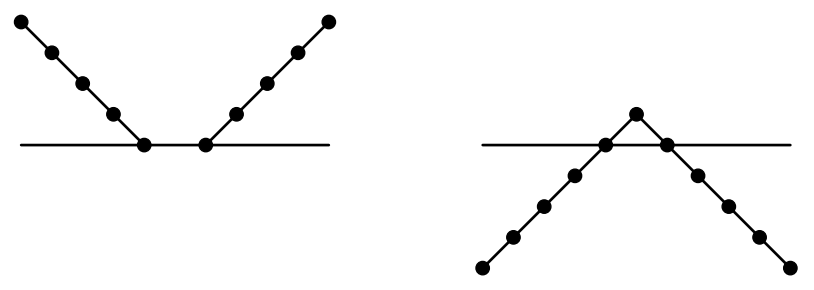

Figure 6. The graph of $Q_{-1}+Q_{1}$ and the graph of $Q_{0}$.

We have $\Phi\left(Z_{\mathcal{S}}\right)=\{-1\} \cup\{0\} \cup\{1\}$. In this case $\Phi^{-1}( \pm 1)=\left\{\left(p_{ \pm}, p_{ \pm}\right)\right\}$, and $\Phi^{-1}(0)$ is not smooth.

Consider the associated decomposition of

$$
\mathcal{Q}_{T}(N, \mathcal{S})=Q_{-1}+Q_{0}+Q_{1}
$$

We have

$$
Q_{-1}=\sum_{k=-\infty}^{-2}(-k-1) t^{k}, \quad Q_{0}=\sum_{k=-\infty}^{-\infty}(|k|-1) t^{k} \quad \text { and } \quad Q_{1}=\sum_{k=2}^{\infty}(k-1) t^{k} .
$$

We see that indeed $Q_{-1}+Q_{0}+Q_{1}=t^{0}$. Figure 6 shows the corresponding multiplicity functions.

\subsection{Some properties of the localized index}

In this subsection, we recall the properties of the localized index $\mathcal{Q}_{K}(M, \mathcal{S}, Z, \Phi)$ that we will use in this article.

\subsubsection{Fixed point submanifolds and $\operatorname{spin}^{c}$-bundles}

Let $\mathcal{S}$ be a $K$-equivariant $\operatorname{spin}^{c}$-bundle over the tangent bundle TM of a $K$-manifold $M$ (equipped with an invariant Riemannian metric). The manifold $M$ is oriented and the Clifford bundle $\mathcal{S}$ is equipped with its canonical $\mathbb{Z} / 2 \mathbb{Z}$-grading. Let $b \in \mathfrak{k}$ be a non-zero $K$ invariant element, and consider the submanifold $M^{b}$ where the vector field $b_{M}$ vanishes. We have an orthogonal decomposition

$$
\left.\mathrm{T} M\right|_{M^{b}}=\mathcal{N} \oplus \mathrm{T} M^{b} .
$$

The normal bundle $\mathcal{N}$ inherits a fibrewise linear endomorphism $\mathcal{L}(b)$ which is antisymmetric relatively to the metric. 
Definition 4.8. - We denote by $\mathcal{N}_{b}$ the vector bundle $\mathcal{N}$ over $M^{b}$ equipped with the complex structure $J_{b}:=\mathcal{L}(b)|\mathcal{L}(b)|^{-1}$.

- We take on $\mathcal{N}$ the orientation $o(\mathcal{N})$ induced by the complex structure $-J_{b}$. On $M^{b}$ we take the orientation $o\left(M^{b}\right)$ defined by $o(\mathcal{N}) o\left(M^{b}\right)=o(M)$.

For further use, we note the following positivity result which follows directly from the definition of $J_{b}$.

Lemma 4.9. Let $m \in M^{b}$.

- The endomorphism $\mathcal{L}(b) / i:\left.\left.\mathcal{N}_{b}\right|_{m} \rightarrow \mathcal{N}_{b}\right|_{m}$ is $\mathbb{C}$-linear, diagonalizable, with positive eigenvalues.

- $\mathcal{L}(b) / i$ acts on the fibers of $\left.\operatorname{det}\left(\mathcal{N}_{b}\right)\right|_{m}$ by multiplication by the positive number $\mathbf{n} \operatorname{Tr}_{\left.\mathcal{N}\right|_{m}}|b|$.

Now we explain how $\operatorname{spin}^{c}$ structures are induced on fixed point submanifolds.

Proposition 4.10. Let $\operatorname{det}(\mathcal{S})$ be the determinant line bundle of the spin ${ }^{c}$ bundle $\mathcal{S}$. There exists an equivariant $\operatorname{spin}^{c}$-bundle $\mathbf{d}_{b}(\mathcal{S})$ on the tangent bundle $\mathrm{T} M^{b}$ with determinant line bundle equal to

$$
\operatorname{det}\left(\mathbf{d}_{b}(\mathcal{S})\right):=\left.\operatorname{det}(\mathcal{S})\right|_{M^{b}} \otimes \operatorname{det}\left(\mathcal{N}_{b}\right)
$$

Proof. The restriction $\left.\mathcal{S}\right|_{M^{b}}$ is a $\operatorname{spin}^{c}$-bundle over the tangent bundle

$$
\left.\mathrm{T} M\right|_{M^{b}}=\mathcal{N} \oplus \mathrm{T} M^{b}
$$

We denote $\overline{\mathcal{N}}_{b}$ the vector bundle $\mathcal{N}$ with the complex structure $-J_{b}$. Let $\bigwedge \overline{\mathcal{N}}_{b}$ be the spinor bundle on $\mathcal{N}$ with its canonical grading: since $o(\mathcal{N})=o\left(-J_{b}\right)$ we have

$$
\left(\bigwedge \overline{\mathcal{N}}_{b}\right)^{ \pm}=\bigwedge^{ \pm} \overline{\mathcal{N}}_{b}
$$

Since $\bigwedge \overline{\mathcal{N}}_{b}$ is a graded $\operatorname{spin}^{c}$-bundle over $\mathcal{N}$, we know that there exists an equivariant $\operatorname{spin}^{c}$ bundle $\mathbf{d}_{b}(\mathcal{S})$ over the tangent bundle $\mathrm{T} M^{b}$ (with its canonical grading) such that

$$
\left.\mathcal{S}\right|_{M^{b}}=\bigwedge \overline{\mathcal{N}}_{b} \otimes \mathbf{d}_{b}(\mathcal{S})
$$

is an isomorphism of graded Clifford modules. At the level of determinant line bundle, we get $\left.\operatorname{det}(\mathcal{S})\right|_{M^{b}}=\operatorname{det}\left(\overline{\mathcal{N}}_{b}\right) \otimes \operatorname{det}\left(\mathbf{d}_{b}(\mathcal{S})\right)$. The identity (4.19) then follows.

Consider the linear action $\left.\mathcal{L}(b)\right|_{\mathbf{d}_{b}(\mathcal{S})}$ of $b$ on the fibers of the $\operatorname{spin}^{c}$-bundle

$$
\mathbf{d}_{b}(\mathcal{S}) \longrightarrow M^{b}
$$


LEMma 4.11. We have $\left.\mathcal{L}(b)\right|_{\mathbf{d}_{b}(\mathcal{S})}=i a \operatorname{Id}_{\mathbf{d}_{b}(\mathcal{S})}$, where

$$
a(m)=\left\langle\Phi_{\mathcal{S}}(m), b\right\rangle+\frac{1}{2} \mathbf{n} \operatorname{Tr}_{\mathrm{T}_{m} M}|b|
$$

is a real-valued locally constant function on $M^{b}$.

Proof. Due to Remark 2.8, we know that $a(m)$ is equal to $\left\langle\Phi_{b}(m), b\right\rangle$, where $\Phi_{b}$ is a moment map attached to the line bundle $\operatorname{det}\left(\mathbf{d}_{b}(\mathcal{S})\right)$. Due to (4.19) we see that $\left\langle\Phi_{b}(m), b\right\rangle=\left\langle\Phi_{\mathcal{S}}(m), b\right\rangle+\frac{1}{2} \mathbf{n} \operatorname{Tr}_{\left.\mathcal{N}\right|_{m}}|b|$. Here we use that $i \mathbf{n} \operatorname{Tr}_{\mathcal{N}_{\mid m} \mid}|b|$ is equal to the complex trace of $\mathcal{L}(b):\left.\left.\operatorname{det}\left(\mathcal{N}_{b}\right)\right|_{m} \rightarrow \operatorname{det}\left(\mathcal{N}_{b}\right)\right|_{m}$ (see Lemma 4.9). The factor $\frac{1}{2}$ is due to the fact that $\Phi_{\mathcal{S}}$ is the moment map of "half" the line bundle $\operatorname{det}(\mathcal{S})$ (see Remark 2.9).

Also, $\mathbf{n T r}_{\mathrm{T}_{m} M}|b|=\mathbf{n} \operatorname{Tr}_{\left.\mathcal{N}\right|_{m}}|b|$ as well as $\left\langle\Phi_{\mathcal{S}}(m), b\right\rangle$ are locally constant on $M^{b}$.

The localization formula of Atiyah-Segal can be expressed in the following way (see $[28, \S 2.2])$.

TheOREM 4.12. Let $b \in \mathfrak{k}$ be a non-zero $K$-invariant element and assume that $M$ is compact. For any complex $K$-vector bundle $\mathcal{W} \rightarrow M$, we have the following equalities in $\widehat{R}(K)$ :

$$
\mathcal{Q}_{K}(M, \mathcal{S} \otimes \mathcal{W})=\mathcal{Q}_{K}\left(M^{b},\left.\mathbf{d}_{b}(\mathcal{S}) \otimes \mathcal{W}\right|_{M^{b}} \otimes \operatorname{Sym}\left(\mathcal{N}_{b}\right)\right) .
$$

Here $\operatorname{Sym}\left(\mathcal{N}_{b}\right)$ is the symmetric algebra of the complex vector bundle $\mathcal{N}_{b}$.

\subsubsection{The localization formula over a coadjoint orbit}

Let $\Phi: M \rightarrow \mathfrak{k}^{*}$ be an equivariant map. Let $\beta \in \mathfrak{k}^{*}$. We also consider $\beta$ as an element of $\mathfrak{k}$ that we denote by the same symbol.

In this section we do not assume that the manifold $M$ is compact but only that $Z_{\beta}=K\left(M^{\beta} \cap \Phi^{-1}(\beta)\right)$ is a compact component of $Z_{\Phi} \subset M$. The study of

$$
\mathcal{Q}_{K}\left(M, \mathcal{S} \otimes \mathcal{W}, Z_{\beta}, \Phi\right) \in \widehat{R}(K)
$$

is thus localized in a neighborhood of $\Phi^{-1}(K \beta)$, an induced manifold. Let us recall the corresponding induction formula.

The restriction of $\Phi$ to $M^{\beta}$ is a $K_{\beta}$-equivariant map taking value in $\mathfrak{k}_{\beta}^{*}$. The subset $Z_{\beta}^{\prime}=M^{\beta} \cap \Phi^{-1}(\beta)$ is a compact component of $Z_{\left.\Phi\right|_{M^{\beta}}}=Z_{\Phi} \cap M^{\beta}$. We may then define the localized index

$$
\mathcal{Q}_{K_{\beta}}\left(M^{\beta},\left.\mathbf{d}_{\beta}(\mathcal{S}) \otimes \mathcal{W}\right|_{M^{\beta}}, Z_{\beta}^{\prime},\left.\Phi\right|_{M^{\beta}}\right) \in \widehat{R}\left(K_{\beta}\right)
$$

where $\mathbf{d}_{\beta}(\mathcal{S})$ is the graded $\operatorname{spin}^{c}$-bundle on $M^{\beta}$ defined in Proposition 4.10. 
We consider the normal bundle $\mathcal{N} \rightarrow M^{\beta}$ of $M^{\beta}$ in $M$. Recall that $\mathcal{N}_{\beta}$ denotes the vector bundle $\mathcal{N}$ equipped with the complex $J_{\beta}$. The following formula is proved in [26] and [28]:

$$
\begin{aligned}
& \mathcal{Q}_{K}\left(M, \mathcal{S} \otimes \mathcal{W}, Z_{\beta}, \Phi\right) \\
& \quad=\operatorname{Ind}_{K_{\beta}}^{K}\left(\mathcal{Q}_{K_{\beta}}\left(M^{\beta},\left.\mathbf{d}_{\beta}(\mathcal{S}) \otimes \mathcal{W}\right|_{M^{\beta}} \otimes \operatorname{Sym}\left(\mathcal{N}_{\beta}\right), Z_{\beta}^{\prime},\left.\Phi\right|_{M^{\beta}}\right) \otimes \bigwedge\left(\mathfrak{k} / \mathfrak{k}_{\beta}\right)_{\mathbb{C}}\right) .
\end{aligned}
$$

Remark 4.13. When $K$ is abelian, this gives

$$
\begin{aligned}
& \mathcal{Q}_{K}\left(M, \mathcal{S} \otimes \mathcal{W}, \Phi^{-1}(\beta) \cap M^{\beta}, \Phi\right) \\
& \quad=\mathcal{Q}_{K}\left(M^{\beta},\left.\mathbf{d}_{\beta}(\mathcal{S}) \otimes \mathcal{W}\right|_{M^{\beta}} \otimes \operatorname{Sym}\left(\mathcal{N}_{\beta}\right), \Phi^{-1}(\beta) \cap M^{\beta},\left.\Phi\right|_{M^{\beta}}\right),
\end{aligned}
$$

which shows that the Atiyah-Segal localization formula (4.12) still holds for the Witten deformation.

Thus we obtain the following proposition.

Proposition 4.14. Let $\mathcal{S}$ be a $K$-equivariant $\operatorname{spin}^{c}$-bundle over $M$, with its canonical grading. Let $\Phi: M \rightarrow \mathfrak{k}^{*}$ be an equivariant map. Let $\mathcal{W} \rightarrow M$ be an equivariant complex vector bundle. Assume that $Z_{\beta}=K\left(M^{\beta} \cap \Phi^{-1}(\beta)\right)$ is a compact component of $Z_{\Phi} \subset M$. Then

$$
\begin{aligned}
& {\left[\mathcal{Q}_{K}\left(M, \mathcal{S} \otimes \mathcal{W}, Z_{\beta}, \Phi\right)\right]^{K}} \\
& \quad=\left[\mathcal{Q}_{K_{\beta}}\left(M^{\beta},\left.\mathbf{d}_{\beta}(\mathcal{S}) \otimes \mathcal{W}\right|_{M^{\beta}} \otimes \operatorname{Sym}\left(\mathcal{N}_{\beta}\right), Z_{\beta}^{\prime},\left.\Phi\right|_{M^{\beta}}\right) \otimes \bigwedge\left(\mathfrak{k} / \mathfrak{k}_{\beta}\right)_{\mathbb{C}}\right]^{K_{\beta}} .
\end{aligned}
$$

This proposition will be used to obtain vanishing results, by studying the infinitesimal action of $\beta$ on the vector bundle $\left.\mathbf{d}_{\beta}(\mathcal{S}) \otimes \mathcal{W}\right|_{M^{\beta}} \otimes \operatorname{Sym}\left(\mathcal{N}_{\beta}\right)$.

The formula (4.21) can be specialized to each connected component of $M^{\beta}$. For a connected component $\mathcal{X} \subset M^{\beta}$ intersecting $\Phi^{-1}(\beta)$, we define the compact subset

$$
Z_{\beta}(\mathcal{X})=K\left(\mathcal{X} \cap \Phi^{-1}(\beta)\right) \subset Z_{\beta}
$$

First we note that $\mathcal{Q}_{K}\left(M, \mathcal{S} \otimes \mathcal{W}, Z_{\beta}, \Phi\right)$ is equal to the $\operatorname{sum} \sum_{\mathcal{X}} \mathcal{Q}_{K}\left(M, \mathcal{S} \otimes \mathcal{W}, Z_{\beta}(\mathcal{X}), \Phi\right)$ parameterized by the connected component of $M^{\beta}$ intersecting $\Phi^{-1}(\beta)$ (they are finite in number).

We have a localization formula for each term $\mathcal{Q}_{K}\left(M, \mathcal{S} \otimes \mathcal{W}, Z_{\beta}(\mathcal{X}), \Phi\right)$ separately (see [26] and [28]):

$$
\begin{aligned}
& {\left[\mathcal{Q}_{K}\left(M, \mathcal{S} \otimes \mathcal{W}, Z_{\beta}(\mathcal{X}), \Phi\right)\right]^{K}} \\
& \quad=\left[\mathcal{Q}_{K_{\beta}}\left(\mathcal{X},\left.\left.\left.\mathbf{d}_{\beta}(\mathcal{S})\right|_{\mathcal{X}} \otimes \mathcal{W}\right|_{\mathcal{X}} \otimes \operatorname{Sym}\left(\mathcal{N}_{\beta}\right)\right|_{\mathcal{X}}, Z_{\beta}^{\prime}(\mathcal{X}),\left.\Phi\right|_{\mathcal{X}}\right) \otimes \bigwedge\left(\mathfrak{k} / \mathfrak{k}_{\beta}\right)_{\mathbb{C}}\right]^{K_{\beta}}
\end{aligned}
$$

where $Z_{\beta}^{\prime}(\mathcal{X})=\mathcal{X} \cap \Phi^{-1}(\beta) \subset Z_{\beta}^{\prime}$. 


\subsubsection{Induction formula}

For the Witten deformation, we proved in $[28, \S 3.3]$ the following variation on the invariance of the index under direct images.

Let $H$ be a closed subgroup of $K$, and consider an $H$-invariant decomposition

$$
\mathfrak{k}=\mathfrak{h} \oplus \mathfrak{q} .
$$

Let $B_{\mathfrak{q}}$ be an open ball in $\mathfrak{q}$, centered at 0 and $H$-invariant. Let $N^{\prime}$ be an $H$-manifold, and consider $N=K \times_{H}\left(B_{\mathfrak{q}} \times N^{\prime}\right)$. Then $N^{\prime}$ is a submanifold of $N$, and the normal bundle of $N^{\prime}$ in $N$ is isomorphic to the trivial bundle with fiber $\mathfrak{q} \oplus \mathfrak{q}$. Let $S_{\mathfrak{q}}$ be the $\operatorname{spin}^{c}$ module for $\mathfrak{q} \oplus \mathfrak{q}$ (we can take $\Lambda \mathfrak{q}_{\mathbb{C}}$ as realization of $S_{\mathfrak{q}}$ ). Thus, if $\mathcal{E}$ is a $K$-equivariant graded Clifford bundle on $N$, there exists an $H$-equivariant graded Clifford bundle $\mathcal{E}^{\prime}$ on $N^{\prime}$ such that

$$
\left.\mathcal{E}\right|_{N^{\prime}}=S_{\mathfrak{q}} \otimes \mathcal{E}^{\prime}
$$

Let $\Phi^{\prime}: N^{\prime} \rightarrow \mathfrak{h}^{*}$ be an $H$-equivariant map, and let $\Phi: N \rightarrow \mathfrak{k}^{*}$ be a $K$-equivariant map. We assume that these maps are linked by the following relations:

$$
\left\{\begin{array}{l}
\left.\Phi\right|_{N^{\prime}}=\Phi^{\prime} \\
\Phi\left(\left[1 ; X, n^{\prime}\right]\right) \in \mathfrak{h}^{*} \text { if and only if } X=0 \\
\left(\Phi\left(\left[1 ; X, n^{\prime}\right]\right), X\right) \geqslant 0
\end{array}\right.
$$

for $\left(X, n^{\prime}\right) \in B_{\mathfrak{q}} \times N^{\prime}$.

Under these conditions, we see that the critical sets $Z_{\Phi} \subset N$ and $Z_{\Phi^{\prime}} \subset N^{\prime}$ are related by $Z_{\Phi}=K \times_{H}\left(\{0\} \times Z_{\Phi^{\prime}}\right)$.

Proposition 4.15. ([28]) Let $Z$ be a compact component of $Z_{\Phi}$ and $Z^{\prime}$ its intersection with $N^{\prime}$. Then $Z^{\prime}$ is a compact component of $Z_{\Phi^{\prime}}$ and

$$
\mathcal{Q}_{K}(N, \mathcal{E}, Z, \Phi)=\operatorname{Ind}_{H}^{K}\left(\mathcal{Q}_{H}\left(N^{\prime}, \mathcal{E}^{\prime}, Z^{\prime}, \Phi^{\prime}\right)\right)
$$

This leads to the relation $\left[\mathcal{Q}_{K}(N, \mathcal{E}, Z, \Phi)\right]^{K}=\left[\mathcal{Q}_{H}\left(N^{\prime}, \mathcal{E}^{\prime}, Z^{\prime}, \Phi^{\prime}\right)\right]^{H}$.

\subsection{The function $d_{\mathcal{S}}$}

Let $M$ be a compact oriented even-dimensional $K$-manifold, equipped with a $K$-equivariant $\operatorname{spin}^{c}$ bundle $\mathcal{S}$. Let $\Phi_{\mathcal{S}}$ be the associated moment map on $M$, and $\varkappa_{\mathcal{S}}$ be the Kirwan vector field. Let $Z_{\mathcal{S}}$ be the vanishing set of $\varkappa_{\mathcal{S}}$ :

$$
Z_{\mathcal{S}}=\left\{m \in M: \Phi_{\mathcal{S}}(m) \cdot m=0\right\}=\bigcup_{\theta}\left(M^{\theta} \cap \Phi_{\mathcal{S}}^{-1}(\theta)\right) .
$$


We now introduce a function $d_{\mathcal{S}}: Z_{\mathcal{S}} \rightarrow \mathbb{R}$ which will localize our study of $\left[\mathcal{Q}_{K}\left(M, \mathcal{S}, Z_{\mathcal{S}}\right)\right]^{K}$ to special components $Z$ of $Z_{\mathcal{S}}$.

Define $d_{\mathcal{S}}: Z_{\mathcal{S}} \rightarrow \mathbb{R}$ by the following relation

$$
d_{\mathcal{S}}(m)=\|\theta\|^{2}+\frac{1}{2} \mathbf{n} \operatorname{Tr}_{\mathrm{T}_{m} M}|\theta|-\mathbf{n} \operatorname{Tr}_{\mathfrak{k}}|\theta|, \quad \text { with } \theta=\Phi_{\mathcal{S}}(m)
$$

LEMma 4.16. - The function $d_{\mathcal{S}}$ is a $K$-invariant locally constant function on $Z_{\mathcal{S}}$ that takes a finite number of values.

- The subsets $Z_{\mathcal{S}}^{>0}=\left\{d_{\mathcal{S}}>0\right\}, Z_{\mathcal{S}}^{=0}=\left\{d_{\mathcal{S}}=0\right\}$ and $Z_{\mathcal{S}}^{<0}=\left\{d_{\mathcal{S}}<0\right\}$ are components of $Z_{\mathcal{S}}$.

Proof. The $K$-invariance of $d_{\mathcal{S}}$ is immediate.

The image $\Phi_{\mathcal{S}}\left(Z_{\mathcal{S}}\right)$ is equal to a finite union $\bigcup_{j} \mathcal{O}_{j}$ of coadjoint orbits. For each coadjoint orbit $\mathcal{O}=K \beta$, the set $Z_{\mathcal{S}} \cap \Phi_{\mathcal{S}}^{-1}(\mathcal{O})$ is equal to a finite disjoint union

$$
\bigcup_{j} K\left(\mathcal{X}^{j} \cap \Phi_{\mathcal{S}}^{-1}(\beta)\right),
$$

where $\left(\mathcal{X}^{j}\right)$ are the connected components of $M^{\beta}$ intersecting $\Phi_{\mathcal{S}}^{-1}(\beta)$. Since the mapping $m \mapsto \mathbf{n} \operatorname{Tr}_{\mathrm{T}_{m} M}|\theta|$ is well defined and locally constant on $M^{\theta}$, the map $d_{\mathcal{S}}$ is constant on each component $K\left(\mathcal{X}^{j} \cap \Phi_{\mathcal{S}}^{-1}(\beta)\right)$. This proves that $d_{\mathcal{S}}$ is locally constant function that takes a finite number of values.

The second point is a direct consequence of the first.

We now prove the following fundamental fact.

Proposition 4.17. Let $Z_{\mathcal{S}}^{>0}$ be the component of $Z_{\mathcal{S}}$ where $d_{\mathcal{S}}$ takes strictly positive values. We have $\left[\mathcal{Q}_{K}\left(M, \mathcal{S}, Z_{\mathcal{S}}^{>0}\right)\right]^{K}=0$.

As $\mathcal{Q}_{K}(M, \mathcal{S})=\mathcal{Q}_{K}\left(M, \mathcal{S}, Z_{\mathcal{S}}^{<0}\right)+\mathcal{Q}_{K}\left(M, \mathcal{S}, Z_{\mathcal{S}}^{=0}\right)+\mathcal{Q}_{K}\left(M, \mathcal{S}, Z_{\mathcal{S}}^{>0}\right)$ by Theorem 4.4, note first the following immediate corollary.

COROLlary 4.18. If $d_{\mathcal{S}}$ takes non-negative values on $Z_{\mathcal{S}}$, we have

$$
\left[\mathcal{Q}_{K}(M, \mathcal{S})\right]^{K}=\left[\mathcal{Q}_{K}\left(M, \mathcal{S}, Z_{\mathcal{S}}^{=0}\right)\right]^{K}
$$

We now prove Proposition 4.17.

Proof. Consider a coadjoint orbit $K \beta$ contained in $\Phi_{\mathcal{S}}\left(Z_{\mathcal{S}}\right)$. Let $\mathcal{X}$ be a connected component of $M^{\beta}$ and let $Z_{\beta}^{\prime}(\mathcal{X}):=\mathcal{X} \cap \Phi^{-1}(\beta)$. Let $Z_{\beta}(\mathcal{X})=K Z_{\beta}^{\prime}(\mathcal{X})$. Let us show that $\left[\mathcal{Q}_{K}\left(M, \mathcal{S}, Z_{\beta}(\mathcal{X})\right)\right]^{K}=0$ if $d_{\mathcal{S}}$ is strictly positive on $Z_{\beta}(\mathcal{X})$.

As $\left[\mathcal{Q}_{K}\left(M, \mathcal{S}, Z_{\beta}(\mathcal{X})\right)\right]^{K}$ is equal to

$$
\left[\mathcal{Q}_{K_{\beta}}\left(\mathcal{X},\left.\left.\mathbf{d}_{\beta}(\mathcal{S})\right|_{\mathcal{X}} \otimes \operatorname{Sym}\left(\mathcal{N}_{\beta}\right)\right|_{\mathcal{X}}, Z_{\beta}^{\prime}(\mathcal{X}),\left.\Phi_{\mathcal{S}}\right|_{\mathcal{X}}\right) \otimes \bigwedge\left(\mathfrak{k} / \mathfrak{k}_{\beta}\right)_{\mathbb{C}}\right]^{K_{\beta}}
$$


by the localization formula (4.22), Lemma 4.1 tells us that the term $\left[\mathcal{Q}_{K}\left(M, \mathcal{S}, Z_{\beta}(\mathcal{X})\right)\right]^{K}$ vanishes if the fibers of the vector bundles

$$
\left.\left.\mathbf{d}_{\beta}(\mathcal{S})\right|_{\mathcal{X}} \otimes \operatorname{Sym}^{j}\left(\mathcal{N}_{\beta}\right)\right|_{\mathcal{X}} \otimes \bigwedge\left(\mathfrak{k} / \mathfrak{k}_{\beta}\right)_{\mathbb{C}}
$$

do not contain non-zero vector killed by the infinitesimal action $\mathcal{L}(\beta)$.

We will prove this fact by showing that the infinitesimal action $\mathcal{L}(\beta) / i$ on the fibers of the vector bundles $\left.\left.\mathbf{d}_{\beta}(\mathcal{S})\right|_{\mathcal{X}} \otimes \operatorname{Sym}^{j}\left(\mathcal{N}_{\beta}\right)\right|_{\mathcal{X}} \otimes \wedge\left(\mathfrak{k} / \mathfrak{k}_{\beta}\right)_{\mathbb{C}}$ have only strictly positive eigenvalues. We establish this by minorizing the possible eigenvalues: they are sums of eigenvalues on each factor of the tensor product.

We have

$$
\frac{1}{i} \mathcal{L}(\beta) \begin{cases}=\|\beta\|^{2}+\frac{1}{2} \mathbf{n} \operatorname{Tr}_{\mathrm{T} M \mid \mathcal{X}}|\beta| & \text { on }\left.\mathbf{d}_{\beta}(\mathcal{S})\right|_{\mathcal{X}} \\ \geqslant 0 & \text { on }\left.\operatorname{Sym}^{j}\left(\mathcal{N}_{\beta}\right)\right|_{\mathcal{X}} \\ \geqslant-\mathbf{n T r}_{\mathfrak{k}}|\beta| & \text { on } \bigwedge\left(\mathfrak{k} / \mathfrak{k}_{\beta}\right)_{\mathbb{C}}\end{cases}
$$

In the first equality, we have used Lemma 4.11: the function $m \mapsto\left\langle\Phi_{\mathcal{S}}(m), \beta\right\rangle$ is constant on $\mathcal{X}$, and as $\mathcal{X}$ contains a point projecting on $\beta$,

$$
\left.\frac{1}{i} \mathcal{L}(\beta)\right|_{\left.\mathbf{d}_{\beta}(\mathcal{S})\right|_{\mathcal{X}}}=\left(\|\beta\|^{2}+\frac{1}{2} \mathbf{n} \operatorname{Tr}_{\left.\mathrm{T} M\right|_{\mathcal{X}}}|\beta|\right) \operatorname{Id}_{\left.\mathbf{d}_{\beta}(\mathcal{S})\right|_{\mathcal{X}}} .
$$

In the second inequality, we used Lemma 4.9 , so that the action of $\mathcal{L}(\beta) / i$ on the graded piece $\operatorname{Sym}^{j}\left(\mathcal{N}_{\beta}\right)$ is strictly positive for $j>0$ or equal to 0 for $j=0$.

In the last inequality, we have used Lemma 3.11.

If $d_{\mathcal{S}}$ takes a strictly positive value on $Z_{\beta}(\mathcal{X})$, we see that $\mathcal{L}(\beta) / i>0$ on

$$
\left.\left.\mathbf{d}_{\beta}(\mathcal{S})\right|_{\mathcal{X}} \otimes \operatorname{Sym}^{j}\left(\mathcal{N}_{\beta}\right)\right|_{\mathcal{X}} \otimes \bigwedge\left(\mathfrak{k} / \mathfrak{k}_{\beta}\right)_{\mathbb{C}}
$$

This forces (4.25) to be equal to zero.

\subsection{The Witten deformation on the product $M \times \mathcal{O}^{*}$}

In this section, $M$ is a compact oriented even-dimensional $K$-manifold, equipped with a $K$-equivariant $\operatorname{spin}^{c}$ bundle $\mathcal{S}$. Let $\Phi_{\mathcal{S}}$ be the associated moment map on $M$. Our aim is to compute geometrically the multiplicities of the equivariant index $\mathcal{Q}_{K}(M, \mathcal{S})$.

\subsubsection{Vanishing theorems}

Let $\mathcal{H}_{\mathfrak{k}}$ be the set of conjugacy classes of the reductive algebras $\mathfrak{k}_{\xi}, \xi \in \mathfrak{k}^{*}$. We denote by $\mathcal{S}_{\mathfrak{k}}$ the set of conjugacy classes of the semi-simple parts $[\mathfrak{h}, \mathfrak{h}]$ of the elements $(\mathfrak{h}) \in \mathcal{H}_{\mathfrak{k}}$. 
Recall that an orbit $\mathcal{P}$ is an (h)-ancestor of $\mathcal{O}$ if $\mathcal{P}$ belongs to the Dixmier sheet $\mathfrak{k}_{(\mathfrak{h})}^{*}$ and $s(\mathcal{P})=\mathcal{O}$. Here $s(\mathcal{P})$ is defined as follows: if $\mathcal{P}=K \mu$ with $\mathfrak{k}_{\mu}=\mathfrak{h}$, then $s(\mathcal{P})=$ $K(\mu+o(\mathfrak{h}))$ (see Definition 3.5).

Recall that the map $\mathcal{O} \mapsto \pi_{\mathcal{O}}:=\mathrm{Q}_{K}^{\text {spin }}(\mathcal{O})$ is a bijection between the regular admissible orbits and $\widehat{K}$. If $\mathcal{O}$ is a regular admissible orbit, then $\mathcal{O}^{*}:=-\mathcal{O}$ is also admissible and $\pi_{\mathcal{O}^{*}}=\left(\pi_{\mathcal{O}}\right)^{*}$. If we apply the shifting trick, we see that the multiplicity of $\pi_{\mathcal{O}}$ in $\mathcal{Q}_{K}(M, \mathcal{S})$ is equal to

$$
\mathrm{m}_{\mathcal{O}}=\left[\mathcal{Q}_{K}(M, \mathcal{S}) \otimes\left(\pi_{\mathcal{O}}\right)^{*}\right]^{K}=\left[\mathcal{Q}_{K}\left(M \times \mathcal{O}^{*}, \mathcal{S} \otimes \mathcal{S}_{\mathcal{O}^{*}}\right)\right]^{K}
$$

Let $\left(\mathfrak{k}_{M}\right)$ be the generic infinitesimal stabilizer of the $K$-action on $M$. In this section, we prove the following vanishing results.

Theorem 4.19. • If $\left(\left[\mathfrak{k}_{M}, \mathfrak{k}_{M}\right]\right) \neq([\mathfrak{h}, \mathfrak{h}])$ for any $(\mathfrak{h}) \in \mathcal{H}_{\mathfrak{k}}$, then

$$
\mathcal{Q}_{K}(M, \mathcal{S})=0
$$

for any $K$-equivariant $\operatorname{spin}^{c}$-bundle $\mathcal{S}$ on $M$.

- Assume that $\left(\left[\mathfrak{k}_{M}, \mathfrak{k}_{M}\right]\right)=([\mathfrak{h}, \mathfrak{h}])$ for $(\mathfrak{h}) \in \mathcal{H}_{\mathfrak{k}}$. Then

$$
\mathrm{m}_{\mathcal{O}}=0
$$

if there is no $(\mathfrak{h})$-ancestor $\mathcal{P}$ to $\mathcal{O}$ contained in $\Phi_{\mathcal{S}}(M)$.

We consider the product $M \times \mathcal{O}^{*}$ equipped with the spin ${ }^{c}$-bundle $\mathcal{S} \otimes \mathcal{S}_{\mathcal{O}^{*}}$. The corresponding moment map is $\Phi_{\mathcal{S} \otimes \mathcal{S}_{\mathcal{O}^{*}}}(m, \xi)=\Phi_{\mathcal{S}}(m)+\xi$. We use the simplified notation

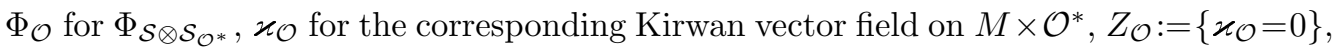
and $d_{\mathcal{O}}$ for the function $d_{\mathcal{S} \otimes \mathcal{S}_{\mathcal{O}^{*}}}$ on $Z_{\mathcal{O}}$. Theorem 4.19 will result from a careful analysis of the function $d_{\mathcal{O}}: Z_{\mathcal{O}} \rightarrow \mathbb{R}$ that was introduced in $\S 4.4$. Due to Proposition 4.17 and Corollary 4.18, Theorem 4.19 is a direct consequence of the following theorem.

THEOREM 4.20. Let $\mathcal{O}$ be a regular admissible orbit.

- The function $d_{\mathcal{O}}$ is non negative on $Z_{\mathcal{O}}$.

- If the function $d_{\mathcal{O}}$ is not strictly positive, then there exists a unique $(\mathfrak{h}) \in \mathcal{H}_{\mathfrak{k}}$ such that the following conditions are satisfied:

(1) $\left(\left[\mathfrak{k}_{M}, \mathfrak{k}_{M}\right]\right)=([\mathfrak{h}, \mathfrak{h}])$.

(2) the orbit $\mathcal{O}$ has an $(\mathfrak{h})$-ancestor $\mathcal{P}$ contained in $\Phi_{\mathcal{S}}(M)$.

Proof. Let $P=M \times \mathcal{O}^{*}$ and let us compute the function $d_{\mathcal{O}}$ on $Z_{\mathcal{O}}$. Let $m \in M$ and $\lambda \in \mathcal{O}$. The point $p=(m,-\lambda) \in Z_{\mathcal{O}} \subset P$ if and only $\Phi_{\mathcal{O}}(p) \cdot p=0$. Let $\beta=\Phi_{\mathcal{O}}(p)$. This means that $\beta$ stabilizes $m$ and $\lambda$, and if $\mu=\Phi_{\mathcal{S}}(m) \in \mathfrak{k}^{*}$, then $\beta=\mu-\lambda$.

We write $\mathrm{T}_{(m,-\lambda)} P=\mathrm{T}_{m} M \oplus \mathrm{T}_{-\lambda} \mathcal{O}^{*}$ and, since $\mathcal{O}^{*}$ is a regular orbit, we have $\mathbf{n} \operatorname{Tr}_{\mathrm{T}_{-\lambda} \mathcal{O}^{*}}|\beta|=\mathbf{n} \operatorname{Tr}_{\mathfrak{k}}|\beta|$. 
We consider a $K_{m}$-invariant decomposition $\mathrm{T}_{m} M=\mathfrak{k} \cdot m \oplus E_{m}$ where $\mathfrak{k} \cdot m \simeq \mathfrak{k} / \mathfrak{k}_{m}$, we obtain $\mathbf{n} \operatorname{Tr}_{\mathrm{T}_{m} M}|\beta|=\mathbf{n} \operatorname{Tr}_{E_{m}}|\beta|+\mathbf{n} \operatorname{Tr}_{\mathfrak{k}}|\beta|-\mathbf{n} \mathbf{T r}_{\mathfrak{k}_{m}}|\beta|$. Thus,

$$
\begin{aligned}
d_{\mathcal{O}}(p) & =\|\beta\|^{2}+\frac{1}{2} \mathbf{n} \operatorname{Tr}_{\mathrm{T}_{(m,-\lambda)} P}|\beta|-\mathbf{n} \operatorname{Tr}_{\mathfrak{k}}|\beta|=\|\beta\|^{2}+\frac{1}{2} \mathbf{n} \operatorname{Tr}_{\mathrm{T}_{m} M}|\beta|-\frac{1}{2} \mathbf{n} \mathbf{T r}_{\mathfrak{k}}|\beta| \\
& =\|\beta\|^{2}+\frac{1}{2} \mathbf{n} \mathbf{T r}_{E_{m}}|\beta|-\frac{1}{2} \mathbf{n} \operatorname{Tr}_{\mathfrak{k}_{m}}|\beta| \geqslant\|\beta\|^{2}+\frac{1}{2} \mathbf{n} \operatorname{Tr}_{E_{m}}|\beta|-\frac{1}{2} \mathbf{n} \mathbf{T r}_{\mathfrak{k}_{\mu}}|\beta| .
\end{aligned}
$$

In the last inequality, we used $\mathfrak{k}_{m} \subset \mathfrak{k}_{\mu}$ as $\mu=\Phi_{\mathcal{S}}(m)$. By Proposition 3.14, we have $\|\beta\|^{2}-\frac{1}{2} \mathbf{n} \operatorname{Tr}_{\mathfrak{k}_{\mu}}|\beta| \geqslant 0$ when $\beta=\mu-\lambda$, as $\lambda$ is very regular (being regular and admissible), and $\beta \in \mathfrak{k}_{\mu} \cap \mathfrak{k}_{\lambda}$. Then the first point follows.

Assume now that there exists a point $p=(m,-\lambda) \in Z_{\mathcal{O}}$ such that $d_{\mathcal{O}}(p)=0$. It implies then that $\|\beta\|^{2}=\frac{1}{2} \mathbf{n} \mathbf{T r}_{\mathfrak{k}_{\mu}}|\beta|$ and $\mathbf{n} \mathbf{T r}_{E_{m}}|\beta|=0$. The first equality implies, due to Proposition 3.14, that $K \mu$ is an admissible orbit such that $s(K \mu)=\mathcal{O}$, and that $-\beta \in o(\mathfrak{h}) \subset[\mathfrak{h}, \mathfrak{h}]^{*}$. We write $-\beta=\varrho^{H}$. Now we have to explain why the condition $\mathbf{n T r}_{E_{m}}\left|\varrho^{H}\right|=0$ implies $\left(\left[\mathfrak{k}_{M}, \mathfrak{k}_{M}\right]\right)=([\mathfrak{h}, \mathfrak{h}])$. Since $\Phi_{\mathcal{S}}(m)=\mu$, we have

$$
\left(\mathfrak{k}_{M}\right) \subset\left(\mathfrak{k}_{m}\right) \subset(\mathfrak{h}) .
$$

Consider the $H$-invariant slice $Y=\Phi_{\mathcal{S}}^{-1}\left(U_{\mu}\right)$ constructed in Proposition 3.16. The product $K Y$ is an invariant neighborhood of $m$ isomorphic to $K \times_{H} Y$. The subspace $E_{m}$ can be taken as the subspace $\mathrm{T}_{m} Y \subset \mathrm{T}_{m} M$. Now the condition $\mathbf{n T r}_{E_{m}}\left|\varrho^{H}\right|=0$ implies that $\varrho^{H}$ acts trivially on the connected component $Y_{m}$ of $Y$ containing $m$. Elements $X \in[\mathfrak{h}, \mathfrak{h}]$ such that $X_{Y_{m}}=0$ form an ideal in $[\mathfrak{h}, \mathfrak{h}]$. Since the ideal generated by $\varrho^{H}$ in $[\mathfrak{h}, \mathfrak{h}]$ is equal to $[\mathfrak{h}, \mathfrak{h}]$, we have proved that $[\mathfrak{h}, \mathfrak{h}]$ acts trivially on $Y_{m}$. As $K Y_{m}$ is an open subset of $M$, we get

$$
([\mathfrak{h}, \mathfrak{h}]) \subset\left(\mathfrak{k}_{M}\right) \text {. }
$$

With (4.28) and (4.29) we get $\left(\left[\mathfrak{k}_{M}, \mathfrak{k}_{M}\right]\right)=([\mathfrak{h}, \mathfrak{h}])$. Finally we have proven that if $d_{\mathcal{O}}$ vanishes at some point $p$, then $\left(\left[\mathfrak{k}_{M}, \mathfrak{k}_{M}\right]\right)=([\mathfrak{h}, \mathfrak{h}])$ for some $(\mathfrak{h}) \in \mathcal{H}_{\mathfrak{k}}$, and there exists an admissible orbit $K \mu \subset \mathfrak{k}_{(\mathfrak{h})}^{*} \cap \Phi_{\mathcal{S}}(M)$ such that $s(K \mu)=\mathcal{O}$.

\subsubsection{Geometric properties}

We summarize here some of the geometric properties enjoyed by $(M, \Phi)$, with $\Phi=\Phi_{\mathcal{S}}$, when $\mathcal{Q}_{K}(M, \mathcal{S})$ is not zero.

Let $(\mathfrak{h}) \in \mathcal{H}_{\mathfrak{k}}$. We choose a representative $\mathfrak{h}$. Let $H$ be the corresponding group and $N_{K}(H)$ be the normalizer of $H$ in $K$. Consider the decomposition $\mathfrak{h}=[\mathfrak{h}, \mathfrak{h}] \oplus \mathfrak{z}$, where $\mathfrak{z}$ is the center of $\mathfrak{h}$. Thus $\mathfrak{z}^{*} \subset \mathfrak{h}^{*}$. Consider the open set

$$
\mathfrak{h}_{0}^{*}=\left\{\xi \in \mathfrak{h}^{*}: \mathfrak{k}_{\xi} \subset \mathfrak{h}\right\}
$$

of $\mathfrak{h}^{*}$. Let $\mathfrak{z}_{0}^{*}=\mathfrak{h}_{0}^{*} \cap \mathfrak{z}^{*}$ be the corresponding open subset of $\mathfrak{z}^{*}$.

We first note the following basic proposition. 
Proposition 4.21. Let $M$ be a K-manifold such that $\left(\left[\mathfrak{k}_{M}, \mathfrak{k}_{M}\right]\right)=([\mathfrak{h}, \mathfrak{h}])$ and let $\Phi: M \rightarrow \mathfrak{k}^{*}$ be an equivariant map. Then

- $\Phi(M) \subset K \mathfrak{z}^{*}$.

- Assume that $\mathcal{Y}:=\Phi^{-1}\left(\mathfrak{h}_{0}^{*}\right)$ is non-empty; then

(a) $\mathcal{Y}$ is a submanifold of $M$ invariant by the action of $N_{K}(H)$, and $[H, H]$ acts trivially on $\mathcal{Y}$.

(b) The image $\Phi(\mathcal{Y})$ is contained in $\mathfrak{z}_{0}^{*}$.

(c) The open subset $K \mathcal{Y}$ is diffeomorphic to $K \times_{N_{K}(H)} \mathcal{Y}$.

Proof. Let us prove the first item. Using our $K$-invariant inner product, we consider $\Phi$ as a map $\Phi: M \rightarrow \mathfrak{k}$. The condition on the infinitesimal stabilizer $\left(\mathfrak{k}_{M}\right)$ gives that $M=$ $K M^{[H, H]}$. If $m \in M^{[H, H]}$, the term $\Phi(m)$ belongs to the Lie algebra $\mathfrak{g}$ of the centralizer subgroup $G:=Z_{K}([H, H])$. But one can easily prove that $\mathfrak{z}$ is a Cartan subalgebra of $\mathfrak{g}$ : hence $\Phi(m)$ is conjugated to an element of $\mathfrak{z}$. This proves the first item.

If $\mathcal{Y}$ is non-empty, the proof that it is a submanifold follows the same line than the proof of Proposition 3.16. The set $K \mathcal{Y}$ is a non-empty open set in $M$ : so on $\mathcal{Y}$ we have $\left(\mathfrak{k}_{M}\right)=\left(\mathfrak{k}_{y}\right)$ on a dense open subset $\mathcal{Y}^{\prime}$. The condition $\left(\left[\mathfrak{k}_{M}, \mathfrak{k}_{M}\right]\right)=([\mathfrak{h}, \mathfrak{h}])$ implies that $\operatorname{dim}[\mathfrak{h}, \mathfrak{h}]=\operatorname{dim}\left[\mathfrak{k}_{y}, \mathfrak{k}_{y}\right]$ on $\mathcal{Y}^{\prime}$, but as $\mathfrak{k}_{y} \subset \mathfrak{k}_{\Phi(y)} \subset \mathfrak{h}$, we conclude that $[\mathfrak{h}, \mathfrak{h}]=\left[\mathfrak{k}_{y}, \mathfrak{k}_{y}\right] \subset \mathfrak{k}_{y}$ on $\mathcal{Y}^{\prime}$ : in other words $[H, H]$ acts trivially on $\mathcal{Y}$, and $[\mathfrak{h}, \mathfrak{h}]=\left[\mathfrak{k}_{y}, \mathfrak{k}_{y}\right]$ for any $y \in \mathcal{Y}$. Furthermore, if $\xi=\Phi(y)$, then $[\mathfrak{h}, \mathfrak{h}]$ acts trivially on $\xi$. So $\xi$ is in the center of $\mathfrak{h}$.

Let us prove that $\pi: K \times_{N_{K}(H)} \mathcal{Y} \rightarrow K \mathcal{Y}$ is one-to-one. If $y_{1}=k y_{2}$, we have $\xi_{1}=k \xi_{2}$ with $\xi_{i}=\Phi\left(y_{i}\right), i=1,2$. As $\Phi(\mathcal{Y}) \subset \mathfrak{z}_{0}^{*}$, the stabilizers of $\xi_{1}$ and $\xi_{2}$ are both equal to $H$. It follows that $k$ belongs to the normalizer of $H$.

The following theorem results directly from Theorem 4.20 and Proposition 4.21. Indeed, in the case where $\mathcal{Q}_{K}(M, \mathcal{S}) \neq\{0\}$, then $\left(\left[\mathfrak{k}_{M}, \mathfrak{k}_{M}\right]\right)=([\mathfrak{h}, \mathfrak{h}])$ for some $(\mathfrak{h}) \in \mathcal{H}_{\mathfrak{k}}$. Furthermore, there exists at least a regular admissible orbit $\mathcal{O}$ such that $m_{\mathcal{O}}$ is non-zero, and consequently there exists orbit $\mathcal{P} \subset \mathfrak{k}_{(\mathfrak{h})}^{*} \cap \Phi_{\mathcal{S}}(M)$.

THEOREM 4.22. Let $M$ be a $K$-manifold and let $\mathcal{S}$ be an equivariant spin $^{c}$-bundle on $M$ with moment map $\Phi_{\mathcal{S}}$. Assume that $\mathcal{Q}_{K}(M, \mathcal{S}) \neq\{0\}$. Then

(1) There exists $(\mathfrak{h}) \in \mathcal{H}_{\mathfrak{k}}$ such that $\left(\left[\mathfrak{k}_{M}, \mathfrak{k}_{M}\right]\right)=([\mathfrak{h}, \mathfrak{h}])$.

(2) If $\mathfrak{z}$ is the center of $\mathfrak{h}$, then $\Phi_{\mathcal{S}}(M) \subset K_{\mathfrak{z}}{ }^{*}$ and the open set $\Phi_{\mathcal{S}}^{-1}\left(K_{\mathfrak{z}}^{*}\right)$ is nonempty.

(3) The group $[H, H]$ acts trivially on the submanifold $\mathcal{Y}=\Phi_{\mathcal{S}}^{-1}\left(\mathfrak{z}_{0}^{*}\right)$.

This condition (1) on the $K$-action is always satisfied in the Hamiltonian setting [21], but not always in the spin setting as can be seen in the following example.

Example 4.23. For $n \geqslant 2$, the sphere $S^{n}$ admits a unique spin structure, equivariant under the action of the group $K:=\operatorname{spin}(n+1)$. The generic stabilizer for the $K$-action is 
isomorphic to the group $K_{M}:=\operatorname{spin}(n)$, and we see that $\left(\mathfrak{k}_{M}\right)=\left(\left[\mathfrak{k}_{M}, \mathfrak{k}_{M}\right]\right)$ is not equal to $([\mathfrak{h}, \mathfrak{h}])$, for any $(\mathfrak{h}) \in \mathcal{H}_{\mathfrak{k}}$.

In the next sections, we will restrict the submanifold $\mathcal{Y}$ to a connected component $C$ of $\mathfrak{h}_{0}^{*}$. We work with the $H$-invariant submanifold $\mathcal{Y}_{C}:=\Phi_{\mathcal{S}}^{-1}(C) \subset \mathcal{Y}$ : here the open subset $K \mathcal{Y}_{C}$ is diffeomorphic to $K \times_{H} \mathcal{Y}_{C}$.

We follow the notations of $\S 3.3$. We denote by $\mathfrak{q}^{C}$ the vector space $\mathfrak{k} / \mathfrak{h}$ equipped with the complex structure $J_{C}$. There exists a unique $H$-equivariant spin ${ }^{c}$-bundle $\mathcal{S}_{\mathcal{Y}_{C}}$ on $\mathcal{Y}_{C}$ such that

$$
\left.\mathcal{S}\right|_{\mathcal{Y}_{C}} \simeq \bigwedge \mathfrak{q}^{C} \otimes \mathcal{S}_{\mathcal{Y}_{C}}
$$

At the level of determinant line bundles we have $\operatorname{det}\left(\mathcal{S}_{\mathcal{Y}_{C}}\right)=\left.\operatorname{det}(\mathcal{S})\right|_{\mathcal{Y}_{C}} \otimes \mathbb{C}_{-2 \varrho_{C}}$, and the corresponding moment map satisfies the relation $\Phi_{\mathcal{Y}_{C}}=\left.\Phi_{\mathcal{S}}\right|_{\mathcal{Y}_{C}}-\varrho_{C}$.

We know already that the subgroup $[H, H]$ acts trivially on the submanifold $\mathcal{Y}_{C}$ (see Theorem 4.22). It acts also trivially on the bundle $\mathcal{S}_{\mathcal{Y}_{C}}$ since the moment map $\Phi_{\mathcal{Y}_{C}}$ takes value in $\mathfrak{z}^{*}$ (see Remark 2.8).

\subsubsection{Localization on $Z_{\overline{\mathcal{O}}}^{0}$}

Let $\mathcal{O}$ be a regular admissible orbit. By Theorem 4.20 and Corollary 4.18, we know that our object of study

$$
m_{\mathcal{O}}=\left[\mathcal{Q}_{K}\left(M \times \mathcal{O}^{*}, \mathcal{S} \otimes \mathcal{S}_{\mathcal{O}^{*}}\right)\right]^{K}
$$

is equal to $\left[\mathcal{Q}_{K}\left(M \times \mathcal{O}^{*}, \mathcal{S} \otimes \mathcal{S}_{\mathcal{O}^{*}}, Z_{\overline{\mathcal{O}}}^{=0}, \Phi_{\mathcal{O}}\right)\right]^{K}$.

Let us give a description of the subset $Z_{\mathcal{O}}^{=0}$ of $Z_{\mathcal{O}} \subset M \times \mathcal{O}^{*}$, where $d_{\mathcal{O}}$ vanishes. We denote by $q: M \times \mathcal{O}^{*} \rightarrow \mathfrak{k}^{*} \oplus \mathfrak{k}^{*}$ the map given by $q(m, \xi)=\left(\Phi_{\mathcal{S}}(m),-\xi\right)$. If $\mu$ belongs to a coadjoint orbit $\mathcal{P}$, and $\xi \in \mu+o\left(\mathfrak{k}_{\mu}\right)$, then $\mathcal{P}$ is an ancestor to the orbit $\mathcal{O}$ of $\xi$.

Definition 4.24. Let $\mathcal{P}$ be a coadjoint orbit.

- Define the following subset of $\mathfrak{k}^{*} \oplus \mathfrak{k}^{*}$ :

$$
R(\mathcal{P})=\left\{(\mu, \xi): \mu \in \mathcal{P} \text { and } \xi \in \mu+o\left(\mathfrak{k}_{\mu}\right)\right\}
$$

- Define $Z_{\mathcal{O}}^{\mathcal{P}}=q^{-1}(R(\mathcal{P})) \subset M \times \mathcal{O}^{*}$.

Proposition 4.25. Assume that $M$ is a $K$-manifold with $\left(\left[\mathfrak{k}_{M}, \mathfrak{k}_{M}\right]\right)=([\mathfrak{h}, \mathfrak{h}])$. Let $\mathcal{S}$ be a K-equivariant $\operatorname{spin}^{c}$-bundle over $M$ with moment map $\Phi_{\mathcal{S}}$. Let $\mathcal{O}$ be a regular admissible coadjoint. Then

$$
Z_{\mathcal{O}}^{=0}=\bigsqcup_{\mathcal{P}} Z_{\mathcal{O}}^{\mathcal{P}}
$$


where the disjoint union is over the set of $(\mathfrak{h})$-ancestors to $\mathcal{O}$. Furthermore, if $\mathcal{P}=K \mu$ $a(\mathfrak{h})$-ancestor to $\mathcal{O}$, the set $Z_{\mathcal{O}}^{\mathcal{P}}$ is equal to

$$
K\left(\Phi_{\mathcal{S}}^{-1}(\mu) \times\left\{-\mu+o\left(\mathfrak{k}_{\mu}\right)^{*}\right\}\right) .
$$

Proof. In the proof of Theorem 4.20, we have seen that, if $d_{\mathcal{O}}(m,-\lambda)=0$, then the element $\mu=\Phi_{\mathcal{S}}(m)$ is such that $\left(\mathfrak{k}_{\mu}\right)=(\mathfrak{h})$ and $\lambda=\beta+\mu$ with $\beta \in o\left(\mathfrak{k}_{\mu}\right)$. So $K \mu$ is an (h) ancestor of $\mathcal{O}$ and $q(m,-\lambda) \in \bigsqcup_{\mathcal{P}} Z_{\mathcal{O}}^{\mathcal{P}}$. This proves the inclusion $Z_{\overline{\mathcal{O}}}^{=0} \subset \bigsqcup_{\mathcal{P}} Z_{\mathcal{O}}^{\mathcal{P}}$.

Conversely, take now $(m,-\xi) \in Z_{\mathcal{O}}^{\mathcal{P}}$ and define $\mu=\Phi_{\mathcal{S}}(m)$. So $K \mu$ is an $(\mathfrak{h})$ ancestor of $\mathcal{O}$ and $\xi=\mu+\beta$ with $\beta \in o\left(\mathfrak{k}_{\mu}\right)$. By $K$-invariance, we may assume that $\mu \in \mathfrak{z}_{0}^{*}$, so $m \in \mathcal{Y}$. We have $T_{m} M=\mathfrak{k} / \mathfrak{k}_{m} \oplus T_{m} \mathcal{Y}$. So

$$
d_{\mathcal{O}}(m,-\xi)=\|\beta\|^{2}-\frac{1}{2} \mathbf{n} \operatorname{Tr}_{\mathfrak{k}_{m}}|\beta|+\frac{1}{2} \mathbf{n} \operatorname{Tr}_{\mathrm{T}_{m} \mathcal{Y}}|\beta|
$$

Since $\beta \in o(\mathfrak{h}) \subset[\mathfrak{h}, \mathfrak{h}]$ acts trivially on $\mathcal{Y}$ by Proposition 4.21 , we have

$$
d_{\mathcal{O}}(m,-\xi)=\left\|\varrho^{H}\right\|^{2}-\frac{1}{2} \mathbf{n T r}_{\mathfrak{k}_{m}}\left|\varrho^{H}\right|
$$

But as $[\mathfrak{h}, \mathfrak{h}] \subset \mathfrak{k}_{m} \subset \mathfrak{h}$, and then $\frac{1}{2} \mathbf{n} \operatorname{Tr}_{\mathfrak{k}_{m}}\left|\varrho^{H}\right|=\frac{1}{2} \mathbf{n} \operatorname{Tr}_{\mathfrak{h}}\left|\varrho^{H}\right|=\left\|\varrho^{H}\right\|^{2}$, finally $d_{\mathcal{O}}(m,-\xi)=0$.

If $K \mu$ is an ancestor of the regular admissible orbit $\mathcal{O}$, then $\mu+o\left(\mathfrak{k}_{\mu}\right)$ is a $K_{\mu}$-orbit contained in $\mathcal{O}$. It follows that the set

$$
Z_{\mathcal{O}}^{\mathcal{P}}:=\left\{(m, \xi) \in M \times \mathcal{O}^{*}: \Phi_{\mathcal{S}}(m) \in \mathcal{P} \text { and }-\xi \in \Phi_{\mathcal{S}}(m)+o\left(\mathfrak{k}_{\Phi_{\mathcal{S}}(m)}\right)\right\}
$$

is equal to $(4.31)$.

At this stage we have proved that

$$
\mathrm{m}_{\mathcal{O}}=\sum_{\mathcal{P}} \mathrm{m}_{\mathcal{O}}^{\mathcal{P}}
$$

where the sum runs over the $(\mathfrak{h})$-ancestor of $\mathcal{O}$ and

$$
\mathrm{m}_{\mathcal{O}}^{\mathcal{P}}=\left[\mathcal{Q}_{K}\left(M \times \mathcal{O}^{*}, \mathcal{S} \otimes \mathcal{S}_{\mathcal{O}^{*}}, Z_{\mathcal{O}}^{\mathcal{P}}, \Phi_{\mathcal{O}}\right)\right]^{K}
$$

In the next section we will go into the computation of the terms $\mathrm{m}_{\mathcal{O}}^{\mathcal{P}}$. We finish this section with the following important fact.

Proposition 4.26. Each individual term $\mathrm{m}_{\mathcal{O}}^{\mathcal{P}}$ is independent of the choice of the moment map $\Phi_{\mathcal{S}}$. 
Proof. Let $\Phi_{\mathcal{S}}^{t}, t \in[0,1]$ be a continuous family of moment maps for $\mathcal{S}$. This gives a family $\Phi_{\mathcal{O}}^{t}(m, \xi):=\Phi_{\mathcal{S}}^{t}(m)+\xi$ for $\mathcal{S} \otimes \mathcal{S}_{\mathcal{O}^{*}}$.

Let $\varkappa_{\mathcal{O}}^{t}$ be the Kirwan vector field associated with $\Phi_{\mathcal{O}}^{t}$, and let $Z_{\mathcal{O}}(t):=\left\{\varkappa_{\mathcal{O}}^{t}=0\right\}$. Recall that $Z_{\mathcal{O}}^{=0}(t)=\bigsqcup_{\mathcal{P}} Z_{\mathcal{O}}^{\mathcal{P}}(t)$, where the union runs over the $(\mathfrak{h})$-ancestors of $\mathcal{O}$, and

$$
Z_{\mathcal{O}}^{\mathcal{P}}(t)=K\left(\left(\Phi_{\mathcal{S}}^{t}\right)^{-1}(\mu) \times\left\{-\mu+o\left(\mathfrak{k}_{\mu}\right)^{*}\right\}\right)
$$

if $\mathcal{P}=K \mu$.

We denote simply by $\sigma^{t}$ the symbol $\sigma\left(M \times \mathcal{O}^{*}, \mathcal{S} \otimes \mathcal{S}_{\mathcal{O}^{*}}, \Phi_{\mathcal{O}}^{t}\right)$. For any $t \in[0,1]$, we consider the quantity $Q_{\mathcal{O}}^{\mathcal{P}}(t) \in \widehat{R}(K)$ which is the equivariant index of $\left.\sigma^{t}\right|_{U_{t}}$, where $U_{t}$ is a (small) neighborhood of $Z_{\mathcal{O}}^{\mathcal{P}}(t)$ such that $U_{t} \cap Z_{\mathcal{O}}(t)=Z_{\mathcal{O}}^{\mathcal{P}}(t)$.

Let us prove that the multiplicity $\mathrm{m}_{\mathcal{O}}^{\mathcal{P}}(t)=\left[Q_{\mathcal{O}}^{\mathcal{P}}(t)\right]^{K}$ is independent of $t$. It is sufficient to prove that $t \mapsto\left[Q_{\mathcal{O}}^{\mathcal{P}}(t)\right]^{K}$ is locally constant: let us show that it is constant in a neighborhood of 0 . We follow the same line of proof that the proof of the independence of the connection of the local piece $\mathcal{Q}_{K}\left(M, \mathcal{S}, \Phi_{\mathcal{S}}^{-1}(\mathcal{O}) \cap Z_{\mathcal{S}}\right)$ of $\mathcal{Q}_{K}(M, \mathcal{S})$ in [28].

Let $U_{0}$ be a neighborhood of $Z_{\mathcal{O}}^{\mathcal{P}}(0)$ such that

$$
\bar{U}_{0} \cap Z_{\mathcal{O}}(0)=Z_{\mathcal{O}}^{\mathcal{P}}(0)
$$

The vector field $\varkappa_{\mathcal{O}}^{0}$ does not vanish on $\partial U_{0}$ : there exists $\varepsilon>0$ so that $\varkappa_{\mathcal{O}}^{t}$ does not vanish on $\partial U_{0}$ for $t \in[0, \varepsilon]$. The family $\left.\sigma^{t}\right|_{U_{0}}, t \in[0, \varepsilon]$ is then a homotopy of transversally elliptic symbols: hence they have the same equivariant index.

LEMMA 4.27. For small $t$, we have

$$
U_{0} \cap Z_{\mathcal{O}}^{=0}(t)=Z_{\mathcal{O}}^{\mathcal{P}}(t)
$$

Indeed, by Proposition $4.25, Z_{\mathcal{\mathcal { O }}}^{0}(t)$ projects by the first projection $\Phi_{\mathcal{S}}^{t}: M \times \mathcal{O}^{*} \rightarrow$ $M \rightarrow \mathfrak{k}^{*}$ to a finite union of coadjoint orbits (the $(\mathfrak{h})$-ancestors to $\mathcal{O}$ ), and $Z_{\mathcal{O}}^{\mathcal{P}}(0)$ projects on $\mathcal{P}$. So, for small $t, U_{0} \cap Z_{\overline{\mathcal{O}}}^{=0}(t)$ is the subset $Z_{\mathcal{O}}^{\mathcal{P}}(t)$ of $Z_{\overline{\mathcal{O}}}^{=0}(t)$ projecting on $\mathcal{P}$.

So, for small $t$, we have the decomposition $U_{0} \cap Z_{\mathcal{O}}(t)=Z_{\mathcal{O}}^{\mathcal{P}}(t) \cup Z_{t}$, where $Z_{t}$ is a component contained in $Z_{\mathcal{O}}^{>0}(t)$. Finally, for small $t$, we have

$$
Q_{\mathcal{O}}^{\mathcal{P}}(0)=\operatorname{Index}_{K}\left(\left.\sigma^{0}\right|_{U_{0}}\right)=\operatorname{Index}_{K}\left(\left.\sigma^{t}\right|_{U_{0}}\right)=Q_{\mathcal{O}}^{\mathcal{P}}(t)+\mathcal{Q}_{K}\left(M \times \mathcal{O}^{*}, \mathcal{S} \otimes \mathcal{S}_{\mathcal{O}^{*}}, Z_{t}, \Phi_{\mathcal{O}}^{t}\right)
$$

As $\left[\mathcal{Q}_{K}\left(M \times \mathcal{O}^{*}, \mathcal{S} \otimes \mathcal{S}_{\mathcal{O}^{*}}, Z_{t}, \Phi_{\mathcal{O}}^{t}\right)\right]^{K}=0$ by Proposition 4.17 , the proof of Proposition 4.26 is completed. 


\subsubsection{Computation of $\mathrm{m}_{\mathcal{O}}^{\mathcal{P}}$}

In this section we compute

$$
\mathrm{m}_{\mathcal{O}}^{\mathcal{P}}:=\left[\mathcal{Q}_{K}\left(M \times \mathcal{O}^{*}, \mathcal{S} \otimes \mathcal{S}_{\mathcal{O}^{*}}, Z_{\mathcal{O}}^{\mathcal{P}}, \Phi_{\mathcal{O}}\right)\right]^{K}
$$

for an (h)-ancestor $\mathcal{P}$ of $\mathcal{O}$.

Let $C$ be a connected component of $\mathfrak{h}_{0}^{*}$ that intersects the orbit $\mathcal{P}$. With the help of Proposition 4.15, we will reduce the computation of $\mathrm{m}_{\mathcal{O}}^{\mathcal{P}}$ to a similar computation where the group $K$ acting on $M$ is replaced with the torus $A_{H}=H /[H, H]$ acting on the slice $\mathcal{Y}_{C}$.

Let us recall the geometric property of the orbits $\mathcal{P}$ and $\mathcal{O}$ that we need for our computation. Let $\mu \in \mathcal{P} \cap C$ be such that $\mathcal{P}=K \mu$ with $K_{\mu}=H$. Let $\lambda \in \mu+o(\mathfrak{h})$. Since $\mathcal{P}$ is an ancestor of the regular admissible orbit $\mathcal{O}$, we have $\mathcal{O}=K \lambda$ and the weight $\lambda-\varrho(\lambda)=\mu-\varrho(\mu) \in \mathfrak{z}^{*}$ defines a character of $H$ (see Lemma 3.8).

Then $Z_{\mathcal{O}}^{\mathcal{P}}$ is equal to $K\left(\Phi_{\mathcal{S}}^{-1}(\mu) \times\left\{-\mu+o(\mathfrak{h})^{*}\right\}\right)$. Here $o(\mathfrak{h})$ is the $\varrho$-orbit for $H$, so $o(\mathfrak{h})=o(\mathfrak{h})^{*}$ and $\mathrm{Q}_{H}^{\mathrm{spin}}\left(o(\mathfrak{h})^{*}\right)$ is the trivial representation of $H$.

Let $\mathcal{Y}_{C}=\Phi_{\mathcal{S}}^{-1}(C)$ be the slice relative to the connected component $C$ (see $\S 4.5 .2$ ). Thus $K \mathcal{Y}_{C}$ is an open neighborhood of $\Phi_{\mathcal{S}}^{-1}(\mathcal{P})$ in $M$ diffeomorphic with $K \times_{H} \mathcal{Y}_{C}$. We see that

$$
Z_{\mathcal{O}}^{\mathcal{P}} \subset\left(K \times_{H} \mathcal{Y}_{C}\right) \times \mathcal{O}^{*} \stackrel{j}{\simeq} K \times_{H}\left(\mathcal{Y}_{C} \times \mathcal{O}^{*}\right),
$$

where the isomorphism $j$ is defined by $\left([k ; y],\left[k^{\prime}\right]\right) \mapsto\left[k ;\left(y,\left[k^{-1} k^{\prime}\right]\right)\right]$.

We consider the $H$-manifold $N^{\prime}:=\mathcal{Y}_{C} \times o(\mathfrak{h})^{*}$ and the $K$-manifold

$$
N=K \times_{H}\left(B_{\mathfrak{q}} \times N^{\prime}\right)=K \times_{H}\left(B_{\mathfrak{q}} \times \mathcal{Y}_{C} \times o(\mathfrak{h})^{*}\right),
$$

where $B_{\mathfrak{q}}$ is a small open ball in $\mathfrak{q}$, centered at 0 and $H$-invariant.

Consider the map $\Psi:(X, \xi) \mapsto \exp (X)(-\mu+\xi)$, from $\mathfrak{q} \times o(\mathfrak{h})^{*}$ into $\mathcal{O}^{*}$. For any $\xi \in$ $o(\mathfrak{h})^{*}$, its differential at $(0, \xi)$ is the isomorphism $(X, Y) \mapsto-\mu \circ \operatorname{ad}(X)-\xi \circ \operatorname{ad}(Y)$ from $\mathfrak{q} \oplus \mathfrak{h} / \mathfrak{h}_{\xi}$ into $\mathfrak{k} / \mathfrak{k}_{\xi}$. Hence, when $B_{\mathfrak{q}}$ is small enough, the map $\Psi$ defines a diffeomorphism between $B_{\mathfrak{q}} \times o(\mathfrak{h})^{*}$ into an $H$-invariant neighborhood of the $H$-orbit $-\mu+o(\mathfrak{h})^{*}$.

Hence a $K$-invariant neighborhood of $Z_{\mathcal{O}}^{\mathcal{P}}$ in $M \times \mathcal{O}^{*}$ is diffeomorphic to $N$. Under this isomorphism, the equivariant map $\Phi_{\mathcal{O}}=\Phi_{\mathcal{S}}+i_{\mathcal{O}^{*}}$ defines a map $\Phi$ on $N$. For $k \in K$, $X \in B_{\mathfrak{q}}, y \in \mathcal{Y}_{C}$ and $\xi \in o(\mathfrak{h})^{*}$, we have

$$
\Phi([k ; X, y, \xi])=k\left(\Phi_{\mathcal{S}}(y)+\exp (X)(-\mu+\xi)\right) .
$$

Under this isomorphism, the set $Z_{\mathcal{O}}^{\mathcal{P}}$ becomes

$$
Z:=K \times_{H}\left(\left\{0_{\mathfrak{q}}\right\} \times \Phi_{\mathcal{S}}^{-1}(\mu) \times o(\mathfrak{h})^{*}\right) \subset Z_{\Phi} .
$$


The map $\Phi: N \rightarrow \mathfrak{k}^{*}$, when restricted to $N^{\prime}$, is the $H$-equivariant map $\Phi^{\prime}: N^{\prime} \rightarrow \mathfrak{h}^{*}$ defined by $\Phi^{\prime}(y, \xi)=\Phi_{\mathcal{S}}(y)-\mu+\xi$. Furthermore, if $B_{\mathfrak{q}}$ is small enough, $\Phi([1 ; X, y, \xi])$ belongs to $\mathfrak{h}^{*}$ if and only $X=0$. As $X \in \mathfrak{q}$, we also see that

$$
(\Phi([1 ; X, y, \xi]), X)=\left(\Phi_{\mathcal{S}}(y), X\right)+(\exp (X)(-\mu+\xi), X)=\left(\Phi_{\mathcal{S}}(y)-\mu+\xi, X\right)=0
$$

for all $(X, y, \xi) \in B_{\mathfrak{q}} \times \mathcal{Y}_{C} \times o(\mathfrak{h})^{*}$. Conditions (4.23) are satisfied. Then Proposition 4.15 tells us that

$$
\mathrm{m}_{\mathcal{O}}^{\mathcal{P}}=\left[\mathcal{Q}_{H}\left(N^{\prime}, \mathcal{S}^{\prime}, Z^{\prime}, \Phi^{\prime}\right)\right]^{H},
$$

where $Z^{\prime}:=Z \cap N^{\prime}=\Phi_{\mathcal{S}}^{-1}(\mu) \times o(\mathfrak{h})^{*}$.

Now we have to explain the nature of the spinor bundle $\mathcal{S}^{\prime}$ over $N^{\prime}=\mathcal{Y}_{C} \times o(\mathfrak{h})^{*}$. Let $\mathcal{S}_{o(\mathfrak{h})^{*}}$ be the canonical $\operatorname{spin}^{c}$-bundle of the orbit $o(\mathfrak{h})^{*}$. Let $\mathcal{S}_{\mathcal{Y}_{C}}$ be the $\operatorname{spin}^{c}$-bundle on $\mathcal{Y}_{C}$ defined by (4.30).

Proposition 4.28. We have $\mathcal{S}^{\prime}=\mathcal{S}_{\mathcal{Y}_{C}}^{\mathcal{P}} \otimes \mathcal{S}_{o(\mathfrak{h})^{*}}$, where $\mathcal{S}_{\mathcal{Y}_{C}}^{\mathcal{P}}=\mathcal{S}_{\mathcal{Y}_{C}} \otimes \mathbb{C}_{-\mu+\varrho(\mu)}$ is a $\operatorname{spin}^{c}$-bundle on $\mathcal{Y}_{C}$. The determinant line bundle of $\mathcal{S}_{\mathcal{Y}_{C}}$ is equal to $\left.\operatorname{det}(\mathcal{S})\right|_{\mathcal{Y}_{C}} \otimes \mathbb{C}_{-2 \mu}$, and the corresponding moment map is $\Phi_{\mathcal{Y}_{C}}^{\mathcal{P}}:=\left.\Phi_{\mathcal{S}}\right|_{\mathcal{Y}_{C}}-\mu$.

The subgroup $[H, H]$ acts trivially on $\left(\mathcal{Y}_{C}, \mathcal{S}_{\mathcal{Y}_{C}}^{\mathcal{P}}\right)$.

Proof. Let $\lambda$ be an element of the $H$-orbit $\mathcal{O}_{\mathcal{P}}:=\mu+o(\mathfrak{h})$. The spinor bundle $\mathcal{S}_{\mathcal{O}^{*}}$ on $\mathcal{O}^{*}=(K \lambda)^{*}$ is

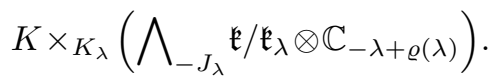

We denote $\mathcal{S}_{1}$ the spinor bundle over $\mathcal{O}_{\mathcal{P}}^{*}$ defined by the relation $\left.\mathcal{S}_{\mathcal{O}^{*}}\right|_{\mathcal{O}_{\mathcal{P}}^{*}} \simeq \bigwedge \overline{\mathfrak{q}^{C}} \otimes \mathcal{S}_{1}$. If we use the identifications $\mathcal{O}_{\mathcal{P}}^{*}=(H \lambda)^{*} \simeq o(\mathfrak{h})^{*}$, we see that $\mathcal{S}_{1}$ is equal to

$$
H \times_{H_{\lambda}}\left(\bigwedge_{-J_{\lambda}} \mathfrak{h} / \mathfrak{h}_{\lambda} \otimes \mathbb{C}_{-\lambda+\varrho(\lambda)}\right) \simeq\left(H \times_{H_{\lambda}} \bigwedge_{-J_{\lambda}} \mathfrak{h} / \mathfrak{h}_{\lambda}\right) \otimes \mathbb{C}_{-\lambda+\varrho(\lambda)} \simeq \mathcal{S}_{o(\mathfrak{h})^{*}} \otimes \mathbb{C}_{-\mu+\varrho(\mu)}
$$

since $\lambda-\varrho(\lambda)=\mu-\varrho(\mu) \in \mathfrak{z}^{*}$ defines a character of $H$ (see Lemma 3.8).

As the spinor bundle $\mathcal{S}_{\mathfrak{q}}$ is equal to the product $\bigwedge \overline{\mathfrak{q}^{C}} \otimes \wedge \mathfrak{q}^{C}$ (see Example 2.3), we know then that $\mathcal{S}^{\prime} \simeq \mathcal{S}_{\mathcal{Y}_{C}} \otimes \mathcal{S}_{1} \simeq \mathcal{S}_{\mathcal{Y}_{C}} \otimes \mathcal{S}_{o(\mathfrak{h})^{*}} \otimes \mathbb{C}_{-\mu+\varrho(\mu)}$.

The relation $\operatorname{det}\left(\mathcal{S}_{\mathcal{Y}_{C}}^{\mathcal{P}}\right)=\left.\operatorname{det}(\mathcal{S})\right|_{\mathcal{Y}_{C}} \otimes \mathbb{C}_{-2 \mu}$ comes from the fact that

$$
\operatorname{det}\left(\mathcal{S}_{\mathcal{Y}_{C}}\right)=\left.\operatorname{det}(\mathcal{S})\right|_{\mathcal{Y}_{C}} \otimes \mathbb{C}_{-2 \varrho_{C}}
$$

and that $\varrho_{C}=\varrho(\mu)$.

We consider now the $H$-manifold $\mathcal{Y}_{C}$ equipped with the $\operatorname{spin}^{c}$-bundle $\mathcal{S}_{\mathcal{Y}_{C}}^{\mathcal{P}}$. Let

$$
\mathcal{Q}_{H}\left(\mathcal{Y}_{C}, \mathcal{S}_{\mathcal{Y}_{C}}^{\mathcal{P}},\{0\}\right) \in \widehat{R}(H)
$$


be the equivariant index localized on the compact component $\left\{\Phi_{\mathcal{Y}_{C}}^{\mathcal{P}}=0\right\}=\left\{\Phi_{\mathcal{S}}=\mu\right\} \subset \mathcal{Y}_{C}$. Let $A_{H}$ be the torus $H /[H, H]$. Since $[H, H]$ acts trivially on $\left(\mathcal{Y}_{C}, \mathcal{S}_{\mathcal{Y}_{C}}\right)$, we may also define the localized index $\mathcal{Q}_{A_{H}}\left(\mathcal{Y}_{C}, \mathcal{S}_{\mathcal{Y}_{C}}^{\mathcal{P}},\{0\}\right) \in \widehat{R}\left(A_{H}\right)$.

We can now prove the main result of this section.

TheOREM 4.29. The multiplicity $\mathrm{m}_{\mathcal{O}}^{\mathcal{P}}$ is equal to

$$
\left[\mathcal{Q}_{H}\left(\mathcal{Y}_{C}, \mathcal{S}_{\mathcal{Y}_{C}}^{\mathcal{P}},\{0\}\right)\right]^{H}=\left[\mathcal{Q}_{A_{H}}\left(\mathcal{Y}_{C}, \mathcal{S}_{\mathcal{Y}_{C}}^{\mathcal{P}},\{0\}\right)\right]^{A_{H}} .
$$

Proof. Let $Z^{\prime}:=\Phi_{\mathcal{S}}^{-1}(\mu) \times o(\mathfrak{h})^{*}$. The character $\mathcal{Q}_{H}\left(N^{\prime}, \mathcal{S}^{\prime}, Z^{\prime}, \Phi^{\prime}\right) \in \widehat{R}(H)$ is equal to the equivariant index of $\sigma\left(N^{\prime}, \mathcal{S}^{\prime}, \Phi^{\prime}\right) \mid \mathcal{U}$, where $\mathcal{U} \subset N^{\prime}$ is an invariant open subset such that $\mathcal{U} \cap Z_{\Phi^{\prime}}=Z^{\prime}$. For $(y, \xi) \in N^{\prime}=\mathcal{Y}_{C} \times o(\mathfrak{h})^{*}$ and $(v, \eta) \in \mathrm{T}_{(y, \xi)} N^{\prime}$, the endomorphism $\left.\sigma\left(N^{\prime}, \mathcal{S}^{\prime}, \Phi^{\prime}\right)\right|_{(y, \xi)}(v, \eta)$ is equal to

$$
\mathbf{c}_{1}\left(v+\left(\Phi_{\mathcal{S}}(y)-\mu+\xi\right) \cdot y\right) \otimes \operatorname{Id}_{\left.\mathcal{S}_{o(\mathfrak{h}) *}\right|_{\xi}}+\varepsilon_{1} \otimes \mathbf{c}_{2}\left(\eta+\left(\Phi_{\mathcal{S}}(y)-\mu+\xi\right) \cdot \xi\right) .
$$

Here $\mathbf{c}_{1}$ acts on $\left.\mathcal{S}_{\mathcal{Y}_{C}}^{\mathcal{P}}\right|_{y}, \mathbf{c}_{2}$ acts on $\left.\mathcal{S}_{o(\mathfrak{h})^{*}}\right|_{\xi}$ and $\varepsilon_{1}$ is the canonical grading operator on $\left.\mathcal{S}_{\mathcal{Y}_{C}}^{\mathcal{P}}\right|_{y}$.

Since $o(\mathfrak{h})^{*}$ is compact, we can replace the term $\mathbf{c}_{2}\left(\eta+\left(\Phi_{\mathcal{S}}(y)-\mu+\xi\right) \cdot \xi\right)$ simply by $\mathbf{c}_{2}(\eta)$. As $[H, H]$ acts trivially on $\mathcal{Y}_{C}$, and $\xi \in[\mathfrak{h}, \mathfrak{h}]$, the vector field $y \mapsto\left(\Phi_{\mathcal{S}}(y)-\mu+\xi\right) \cdot y$ is equal to $y \mapsto\left(\Phi_{\mathcal{S}}(y)-\mu\right) \cdot y$. Thus our symbol is homotopic to the symbol

$$
\left.\mathbf{c}_{1}\left(v+\left(\Phi_{\mathcal{S}}(y)-\mu\right) \cdot y\right) \otimes \operatorname{Id}_{\mathcal{S}_{o(\mathfrak{h})}}\right|_{\xi}+\varepsilon_{1} \otimes \mathbf{c}_{2}(\eta) .
$$

This last expression is the product symbol of the $H$-transversally elliptic symbol $\mathbf{c}_{1}\left(v+\left(\Phi_{\mathcal{S}}(y)-\mu\right) \cdot y\right)$ on $\mathcal{Y}_{C}$ and of the elliptic symbol $\mathbf{c}_{2}(\eta)$ on $o(\mathfrak{h})^{*}$. The equivariant indices multiply under the product (as one is elliptic) ([1],[27]).

Now the $H$-equivariant index of $\mathbf{c}_{2}(\eta)$ acting on $\mathcal{S}_{o(\mathfrak{h})^{*}}$ is the trivial representation of $H$. Thus we obtain our theorem. We also have to remark that the identity $\left[\mathcal{Q}_{H}\left(\mathcal{Y}_{C}, \mathcal{S}_{\mathcal{Y}_{C}}^{\mathcal{P}},\{0\}\right)\right]^{H}=\left[\mathcal{Q}_{A_{H}}\left(\mathcal{Y}_{C}, \mathcal{S}_{\mathcal{Y}_{C}}^{\mathcal{P}},\{0\}\right)\right]^{A_{H}}$ follows from the fact that $[H, H]$ acts trivially on $\left(\mathcal{Y}_{C}, \mathcal{S}_{\mathcal{Y}_{C}}\right)$.

\section{Multiplicities and reduced spaces}

In this section, we interpret the multiplicity as an equivariant index on a reduced space.

Let $\mathcal{O} \subset \mathfrak{k}^{*}$ be a regular admissible orbit, and $(\mathfrak{h}) \in \mathcal{H}_{\mathfrak{k}}$ be such that $([\mathfrak{h}, \mathfrak{h}])=\left(\left[\mathfrak{k}_{M}, \mathfrak{k}_{M}\right]\right)$. In the previous section, we have proved that the multiplicity of $\pi_{\mathcal{O}}$ in $\mathcal{Q}_{K}(M, \mathcal{S})$ is equal to

$$
\mathrm{m}_{\mathcal{O}}=\sum_{\mathcal{P}} \mathrm{m}_{\mathcal{O}}^{\mathcal{P}}
$$


where the sum runs over the $K$-orbits $\mathcal{P}$ which are (h)-ancestors of $\mathcal{O}$. Furthermore, we have proved that $\mathrm{m}_{\mathcal{O}}^{\mathcal{P}}=\left[\mathcal{Q}_{A_{H}}\left(\mathcal{Y}_{C}, \mathcal{S}_{\mathcal{Y}_{C}}^{\mathcal{P}},\{0\}\right)\right]^{A_{H}}$.

The aim of this section is to prove the following theorem.

TheOREM 5.1. The multiplicity $\mathrm{m}_{\mathcal{O}}^{\mathcal{P}}$ is equal to the spin ${ }^{c}$ index of the (possibly singular) reduced space $M_{\mathcal{P}}:=\Phi_{\mathcal{S}}^{-1}(\mathcal{P}) / K$.

However, our first task is to give a meaning to a $\mathrm{Q}^{\text {spin }}\left(M_{\mathcal{P}}\right) \in \mathbb{Z}$, even if $M_{\mathcal{P}}$ is singular.

\section{1. $\operatorname{Spin}^{c}$ index on singular reduced spaces}

We consider a connected oriented manifold $N$, equipped with a $\operatorname{spin}^{c}$-bundle $\mathcal{S}$. We assume that a torus $G$ acts on the data $(N, \mathcal{S})$. An invariant connection on the determinant line bundle $\operatorname{det}(\mathcal{S})$ defines a moment map $\Phi: N \rightarrow \mathfrak{g}^{*}$. We do not assume that $N$ is compact, but we assume that the map $\Phi$ is proper. $\left({ }^{5}\right)$ Thus, for any $\xi \in \mathfrak{g}^{*}$, the reduced space $N_{\xi}:=\Phi^{-1}(\xi) / G$ is compact.

The purpose of this subsection is to explain how we can define the $\operatorname{spin}^{c}$-index, $\mathrm{Q}^{\text {spin }}\left(N_{\mu}\right) \in \mathbb{Z}$, for any $\mu$ in the weight lattice $\Lambda$ of the torus $G$.

Let $\mathfrak{g}_{N}$ be the generic infinitesimal stabilizer of the $G$-action on $N$ : the image of $N$ under the map $\Phi$ lives in an affine space $I(N)$ parallel to $\mathfrak{g}_{N}^{\perp}$. If $\xi \in I(N)$ is a regular value of $\Phi: N \rightarrow I(N)$, the reduced space $N_{\xi}$ is a compact orbifold (as proved in [28]). We can define $\operatorname{spin}^{c}$-bundles on orbifolds, as well as $\operatorname{spin}^{c}$-indices.

We start with the following basic fact.

Lemma 5.2. For any regular value $\xi \in I(N)$ of $\Phi: N \rightarrow I(N)$, the orbifold $N_{\xi}$ is oriented and equipped with a family of $\operatorname{spin}^{c}$-bundles $\mathcal{S}_{\xi}^{\mu}$ parameterized by $\mu \in \Lambda \cap I(N)$.

Proof. Let $G_{N}$ be the subtorus with Lie algebra $\mathfrak{g}_{N}$. Let $G^{\prime}=G / G_{N}$. The dual of the Lie algebra $\mathfrak{g}^{\prime}$ of $G^{\prime}$ admits a canonical identification with $\mathfrak{g}_{N}^{\perp}$.

We assume that $\xi$ is a regular value of $\Phi: N \rightarrow I(N)$ : the fiber $Z=\Phi^{-1}(\xi)$ is a submanifold equipped with a locally free action of $G^{\prime}$. Let $N_{\xi}:=Z / G^{\prime}$ be the corresponding "reduced" space, and let $\pi: Z \rightarrow N_{\xi}$ be the projection map. We can define the tangent (orbi-)bundle $\mathrm{T} N_{\xi}$ to $N_{\xi}$.

On $Z$, we obtain an exact sequence $\left.0 \longrightarrow \mathrm{T} Z \longrightarrow \mathrm{T} N\right|_{Z} \stackrel{d \Phi_{\varepsilon}}{\longrightarrow} Z \times\left(\mathfrak{g}^{\prime}\right)^{*} \rightarrow 0$, and an orthogonal decomposition $\mathrm{T} Z=\mathrm{T}_{G^{\prime}} Z \oplus \mathfrak{g}_{Z}^{\prime}$, where $\mathfrak{g}_{Z}^{\prime}$ is the trivial bundle corresponding to the subspace of $\mathrm{T} Z$ formed by the vector fields generated by the infinitesimal action

$\left({ }^{5}\right)$ We will sometimes use a slightly different hypothesis: $\Phi$ is proper as a map from $N$ to an open subset of $\mathfrak{g}^{*}$. 
of $\mathfrak{g}^{\prime}$. So $\left.\mathrm{T} N\right|_{Z}$ admits the decomposition $\left.\mathrm{T} N\right|_{Z} \simeq \mathrm{T}_{G^{\prime}} Z \oplus \mathfrak{g}_{Z}^{\prime} \oplus\left[\left(\mathfrak{g}^{\prime}\right)^{*}\right]$. We rewrite this as

$$
\left.\mathrm{T} N\right|_{Z} \simeq \mathrm{T}_{G^{\prime}} Z \oplus\left[\mathfrak{g}_{\mathbb{C}}^{\prime}\right]
$$

with the convention $\mathfrak{g}_{Z}^{\prime}=Z \times\left(\mathfrak{g}^{\prime} \otimes i \mathbb{R}\right)$ and $Z \times\left(\mathfrak{g}^{\prime}\right)^{*}=Z \times\left(\mathfrak{g}^{\prime} \otimes \mathbb{R}\right)$. Note that the bundle $\mathrm{T}_{G^{\prime}} Z$ is naturally identified with $\pi^{*}\left(\mathrm{~T} N_{\xi}\right)$.

If we take on $\mathfrak{g}_{\mathbb{C}}^{\prime}$ the orientation $o(i)$ given by the complex structure, there exists a unique orientation $o\left(N_{\xi}\right)$ on $N_{\xi}$ such that $o(N)=o\left(N_{\xi}\right) o(i)$.

Definition 5.3. Let $\widetilde{\mathcal{S}}_{\xi}$ be the $\operatorname{spin}^{c}$-bundle on the vector bundle $\mathrm{T}_{G^{\prime}} Z \rightarrow Z$ such that

$$
\left.\mathcal{S}\right|_{Z} \simeq \widetilde{\mathcal{S}}_{\xi} \otimes\left[\bigwedge \mathfrak{g}_{\mathbb{C}}^{\prime}\right]
$$

The Kostant relation shows that, for any $X \in \mathfrak{g}_{N}$, the element $e^{X}$ acts on the fibers of $\widetilde{\mathcal{S}}_{\xi}$ as a multiplication by $e^{i\langle\nu, X\rangle}$, where $\nu$ is any element of $I(N)$. Hence, for any $\mu \in \Lambda \cap I(N)$, the action of $G_{N}$ on the tensor $\widetilde{\mathcal{S}}_{\xi} \otimes\left[\mathbb{C}_{-\mu}\right]$ is trivial. We can then define a $\operatorname{spin}^{c}$-bundle $\mathcal{S}_{\xi}^{\mu}$ on $\mathrm{T} N_{\xi}$ by the relation

$$
\widetilde{\mathcal{S}}_{\xi} \otimes\left[\mathbb{C}_{-\mu}\right]=\pi^{*}\left(\mathcal{S}_{\xi}^{\mu}\right)
$$

The proof of the following theorem is given in the next subsection.

TheOREM 5.4. For any $\mu \in I(N) \cap \Lambda$, consider the compact oriented orbifold $N_{\mu+\varepsilon}$ associated with a generic $\left({ }^{6}\right)$ element $\varepsilon \in \mathfrak{g}_{N}^{\perp}$. Then the index

$$
\mathcal{Q}\left(N_{\mu+\varepsilon}, \mathcal{S}_{\mu+\varepsilon}^{\mu}\right)
$$

is independent of the choice of a generic and small enough $\varepsilon$.

Due to the previous theorem, one defines the $\operatorname{spin}^{c}$-index of singular reduced spaces as follows.

Definition 5.5. If $\mu \in \Lambda$, the number $\mathrm{Q}^{\operatorname{spin}}\left(N_{\mu}\right)$ is defined by the following dichotomy:

$$
\mathrm{Q}^{\mathrm{spin}}\left(N_{\mu}\right)= \begin{cases}0, & \text { if } \mu \notin I(N), \\ \mathcal{Q}\left(N_{\mu+\varepsilon}, \mathcal{S}_{\mu+\varepsilon}^{\mu}\right), & \text { if } \mu \in I(N) \text { and } \varepsilon \in \mathfrak{g}_{N}^{\perp} \text { is generic and small enough. }\end{cases}
$$

Remark 5.6. The invariant $\mathrm{Q}^{\text {spin }}\left(N_{\mu}\right) \in \mathbb{Z}$ vanishes if $\mu$ does not belong to the relative interior of $\Phi(N)$ in the affine space $I(N)$. It is due to the fact that we can then approach $\mu$ by elements $\mu+\varepsilon$ that are not in the image $\Phi(N)$.

$\left({ }^{6}\right)$ So that $\mu+\varepsilon$ is a regular value of $\Phi: N \rightarrow I(N)$. 
Let us consider the particular case when $\mu \in I(N) \cap \Lambda$ is a regular value of $\Phi: N \rightarrow I(N)$ such that the reduced space $N_{\mu}$ is reduced to a point. Let $m_{o} \in \Phi^{-1}(\mu)$, and let $\Gamma \subset G^{\prime}$ be the stabilizer subgroup of $m_{o}$ ( $\Gamma$ is finite). In this case (5.35) becomes $\mathrm{T}_{m_{o}} N \simeq \mathfrak{g}_{\mathbb{C}}^{\prime}$, and $o\left(N_{\mu}\right)$ is the quotient between the orientation of $N$ and those of $\mathfrak{g}_{\mathbb{C}}^{\prime}$. At the level of graded $\operatorname{spin}^{c}$-bundles, we have

$$
\left.\left.\mathcal{S}\right|_{m_{o}} \simeq o\left(N_{\mu}\right) \bigwedge \mathfrak{g}_{\mathbb{C}}^{\prime} \otimes \operatorname{det}(\mathcal{S})\right|_{m_{o}} ^{1 / 2},
$$

where $\left.\operatorname{det}(\mathcal{S})\right|_{m_{o}} ^{1 / 2}$ is a 1-dimensional representation of $\Gamma$ such that

$$
\left(\left.\operatorname{det}(\mathcal{S})\right|_{m_{o}} ^{1 / 2}\right)^{\otimes 2}=\left.\operatorname{det}(\mathcal{S})\right|_{m_{o}} .
$$

In this case Definition 5.5 becomes

$$
\mathrm{Q}^{\mathrm{spin}}\left(N_{\mu}\right)=o\left(N_{\mu}\right) \operatorname{dim}\left[\left.\operatorname{det}(\mathcal{S})\right|_{m_{o}} ^{1 / 2} \otimes \mathbb{C}_{-\mu}\right]^{\Gamma} \in\{-1,0,1\}
$$

\subsection{Proof of Theorem 5.4}

In this subsection we work with a fixed $\mu \in I(N) \cap \Lambda$. For any $\varepsilon \in \mathfrak{g}(N)^{\perp}$, we consider the moment map $\Phi_{\varepsilon}=\Phi-\mu-\varepsilon$. We denote by $\varkappa$ and $\varkappa_{\varepsilon}$ the Kirwan vector fields attached to the moment maps $\Phi_{0}$ and $\Phi_{\varepsilon}$, respectively.

We start with the fundamental lemma.

LeMmA 5.7. The map $\varepsilon \mapsto\left[\mathcal{Q}_{G}\left(N, \mathcal{S}, \Phi_{\varepsilon}^{-1}(0), \Phi_{\varepsilon}\right) \otimes \mathbb{C}_{-\mu}\right]^{G}$ is constant in a neighborhood of zero.

Proof. Changing $\mathcal{S}$ to $\mathcal{S} \otimes\left[\mathbb{C}_{-\mu}\right]$, we might as well take $\mu=0$.

Let $r>0$ be smallest non-zero critical value of $\|\Phi\|^{2}$, and let $\mathcal{U}:=\Phi^{-1}\left(\left\{\xi\|\xi\|<\frac{1}{2} r\right\}\right)$. Using Remark 2.11, we have $\mathcal{U} \cap\left\{\varkappa_{0}=0\right\}=\Phi^{-1}(0)$.

We describe now $\left\{\varkappa_{\varepsilon}=0\right\} \cap \mathcal{U}$ using a parametrization similar to those introduced in $[25, \S 6]$.

Let $\mathfrak{g}_{i}, i \in I$, be the finite collection of infinitesimal stabilizers for the $G$-action on the compact set $\overline{\mathcal{U}}$. Let $\mathcal{D}$ be the subset of the collection of subspaces $\mathfrak{g}_{i}^{\perp}$ of $\mathfrak{g}^{*}$ such that $\Phi^{-1}(0) \cap N^{\mathfrak{g}_{i}} \neq \varnothing$.

Note that $\mathcal{D}$ is reduced to $I(N)$ if 0 is a regular value of $\Phi: N \rightarrow I(N)$. If $\Delta=\mathfrak{g}_{i}^{\perp}$ belongs to $\mathcal{D}$, and $\varepsilon \in I(N)$, write the orthogonal decomposition $\varepsilon=\varepsilon_{\Delta}+\beta_{\Delta}$ with $\varepsilon_{\Delta} \in \Delta$, and $\beta_{\Delta} \in \mathfrak{g}_{i}$. Let

$$
\mathcal{B}_{\varepsilon}=\left\{\beta_{\Delta}=\varepsilon-\varepsilon_{\Delta}: \Delta \in \mathcal{D}\right\}
$$

be the set of $\beta$ so obtained. 


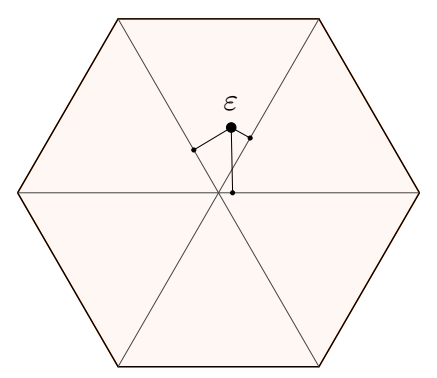

Figure 7. The point $\varepsilon$ and its projections $\varepsilon_{\Delta}$.

We denote by $Z_{\varepsilon}$ the zero set of the vector field $\varkappa_{\varepsilon}$ associated with $\Phi_{\varepsilon}$. Thus, if $\varepsilon$ is sufficiently small $\left(\|\varepsilon\|<\frac{1}{2} r\right)$,

$$
Z_{\varepsilon} \cap \mathcal{U}=\bigcup_{\beta \in \mathcal{B}_{\varepsilon}} N^{\beta} \cap \Phi_{\varepsilon}^{-1}(\beta)
$$

With (5.37) in hands, we easily see that $\left.t \in[0,1] \mapsto \sigma\left(N, \mathcal{S}, \Phi_{t \varepsilon}\right)\right|_{\mathcal{U}}$ is a homotopy of transversally elliptic symbols on $\mathcal{U}$. Hence they have the same index

$$
\mathcal{Q}_{G}\left(\mathcal{U}, \mathcal{S}, \Phi^{-1}(0), \Phi\right)=\mathcal{Q}_{G}\left(\mathcal{U}, \mathcal{S}, Z_{\varepsilon} \cap \mathcal{U}, \Phi_{\varepsilon}\right)=\sum_{\beta \in \mathcal{B}_{\varepsilon}} \mathcal{Q}_{G}\left(N, \mathcal{S}, \Phi_{\varepsilon}^{-1}(\beta) \cap N^{\beta}, \Phi_{\varepsilon}\right) .
$$

The lemma will be proved if we check that $\left[\mathcal{Q}_{G}\left(N, \mathcal{S}, \Phi_{\varepsilon}^{-1}(\beta) X \cap N^{\beta}, \Phi_{\varepsilon}\right)\right]^{G}=0$ for any non-zero $\beta \in \mathcal{B}_{\varepsilon}$.

We fix now a non-zero $\beta:=\beta_{\Delta} \in \mathcal{B}_{\varepsilon}$. The Atiyah-Segal localization formula for the Witten deformation (Remark 4.13) gives

$$
\begin{aligned}
\mathcal{Q}_{G}\left(N, \mathcal{S}, \Phi_{\varepsilon}^{-1}(\beta) \cap N^{\beta}, \Phi_{\varepsilon}\right) & =\mathcal{Q}_{G}\left(N^{\beta}, \mathbf{d}_{\beta}(\mathcal{S}) \otimes \operatorname{Sym}\left(\mathcal{V}_{\beta}\right), \Phi_{\varepsilon}^{-1}(\beta), \Phi_{\varepsilon}\right) \\
& =\sum_{\mathcal{X} \subset N^{\beta}} \mathcal{Q}_{G}\left(\mathcal{X},\left.\left.\mathbf{d}_{\beta}(\mathcal{S})\right|_{\mathcal{X}} \otimes \operatorname{Sym}\left(\mathcal{V}_{\beta}\right)\right|_{\mathcal{X}}, \Phi_{\varepsilon}^{-1}(\beta), \Phi_{\varepsilon}\right),
\end{aligned}
$$

where $\mathcal{V}_{\beta} \rightarrow N^{\beta}$ is the normal bundle of $N^{\beta}$ in $N$ and the sum runs over the connected components $\mathcal{X}$ of $N^{\beta}$ that intersect $\Phi_{\varepsilon}^{-1}(\beta)$. Like in the proof of Proposition 4.17, we will show that the term $\left[\mathcal{Q}_{G}\left(N, \mathcal{S}, \Phi_{\varepsilon}^{-1}(\beta) \cap N^{\beta}, \Phi_{\varepsilon}\right)\right]^{G}$ vanishes by looking to the infinitesimal action of $\beta$, denoted by $\mathcal{L}(\beta)$, on the fibers of the vector bundle $\left.\left.\mathbf{d}_{\beta}(\mathcal{S})\right|_{\mathcal{X}} \otimes \operatorname{Sym}\left(\mathcal{N}_{\beta}\right)\right|_{\mathcal{X}}$.

Let $n \in \Phi_{\varepsilon}^{-1}(\beta) \cap \mathcal{X}$ : we have $\Phi(n)=\beta+\varepsilon=\varepsilon_{\Delta}$, and therefore $\langle\Phi(n), \beta\rangle=\left\langle\varepsilon_{\Delta}, \beta_{\Delta}\right\rangle=0$. Lemma 4.11 tell us that $\left.\mathcal{L}(\beta)\right|_{\mathbf{d}_{b}(\mathcal{S}) \mid \mathcal{X}}=i a \operatorname{Id}_{\mathbf{d}_{b}(\mathcal{S}) \mid \mathcal{X}}$, where

$$
a=\langle\Phi(n), \beta\rangle+\frac{1}{2} \mathbf{n} \operatorname{Tr}_{\mathrm{T}_{n} N}|\beta|=\frac{1}{2} \mathbf{n} \operatorname{Tr}_{\mathrm{T} \mid \mathcal{X}}|\beta| .
$$


We obtain

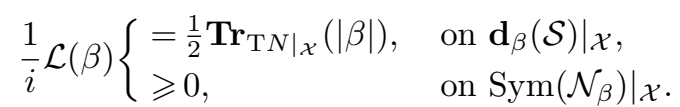

So we have checked that $\mathcal{L}(\beta) / i \geqslant \frac{1}{2} \operatorname{Tr}_{\mathrm{T} N \mid \mathcal{X}}(|\beta|)$ on $\left.\left.\mathbf{d}_{\beta}(\mathcal{S})\right|_{\mathcal{X}} \otimes \operatorname{Sym}\left(\mathcal{N}_{\beta}\right)\right|_{\mathcal{X}}$.

Now we remark that $\beta$ does not act trivially on $N$, since $\beta$ belongs to the direction of the subspace $I(N)=\mathfrak{g}_{N}^{\perp}$ : this forces $\operatorname{Tr}_{\mathrm{T} N \mid \mathcal{X}}(|\beta|)$ to be strictly positive. Finally, we see that $\mathcal{L}(\beta) / i>0$ on $\left.\left.\mathbf{d}_{\beta}(\mathcal{S})\right|_{\mathcal{X}} \otimes \operatorname{Sym}\left(\mathcal{N}_{\beta}\right)\right|_{\mathcal{X}}$, and then

$$
\left[\mathcal{Q}_{G}\left(\mathcal{X},\left.\left.\mathbf{d}_{\beta}(\mathcal{S})\right|_{\mathcal{X}} \otimes \operatorname{Sym}\left(\mathcal{V}_{\beta}\right)\right|_{\mathcal{X}}, \Phi_{\varepsilon}^{-1}(\beta), \Phi_{\varepsilon}\right)\right]^{G}=0
$$

The Lemma 5.7 is proved.

The proof of Theorem 5.4 will be completed with the following lemma.

LEMMA 5.8. If $\mu+\varepsilon$ is a regular value of $\Phi: N \rightarrow I(N)$, the invariant

$$
\left[\mathcal{Q}_{G}\left(N, \mathcal{S}, \Phi_{\varepsilon}^{-1}(0), \Phi_{\varepsilon}\right) \otimes \mathbb{C}_{-\mu}\right]^{G}
$$

is equal to the index $\mathcal{Q}\left(N_{\mu+\varepsilon}, \mathcal{S}_{\mu+\varepsilon}^{\mu}\right)$.

We assume that $\mu+\varepsilon$ is a regular value of $\Phi: N \rightarrow I(N)$ : the fiber $Z=\Phi^{-1}(\mu+\varepsilon)$ is a submanifold equipped with a locally free action of $G^{\prime}=G / G_{N}$. Let $N_{\mu+\varepsilon}:=Z / G^{\prime}$ be the corresponding "reduced" space, and let $\pi: Z \rightarrow N_{\mu+\varepsilon}$ be the projection map. We have the decomposition

$$
\left.\mathrm{T} N\right|_{Z} \simeq \pi^{*}\left(\mathrm{~T} N_{\mu+\varepsilon}\right) \oplus\left[\mathfrak{g}_{\mathbb{C}}^{\prime}\right]
$$

For any $\nu \in \Lambda \cap I(N), \mathcal{S}_{\mu+\varepsilon}^{\nu}$ is the spinor bundle on $N_{\mu+\varepsilon}$ defined by the relation

$$
\left.\mathcal{S}\right|_{Z} \otimes \mathbb{C}_{-\nu} \simeq \pi^{*}\left(\mathcal{S}_{\mu+\varepsilon}^{\nu}\right) \otimes\left[\bigwedge \mathfrak{g}_{\mathbb{C}}^{\prime}\right]
$$

The following result is proved in [28, §2.3].

Proposition 5.9. We have the following equality in $\widehat{R}(G)$ :

$$
\mathcal{Q}_{G}\left(N, \mathcal{S}, \Phi_{\varepsilon}^{-1}(0), \Phi_{\varepsilon}\right)=\sum_{\nu \in \Lambda \cap I(N)} \mathcal{Q}\left(N_{\mu+\varepsilon}, \mathcal{S}_{\mu+\varepsilon}^{\nu}\right) \mathbb{C}_{\nu}
$$

In particular $\left[\mathcal{Q}_{G}\left(N, \mathcal{S}, \Phi_{\varepsilon}^{-1}(0), \Phi_{\varepsilon}\right) \otimes \mathbb{C}_{-\mu}\right]^{G}$ is equal to $\mathcal{Q}\left(N_{\mu+\varepsilon}, \mathcal{S}_{\mu+\varepsilon}^{\mu}\right)$. 


\section{3. $[Q, R]=0$}

We come back to the setting of a compact $K$-manifold $M$, oriented and of even dimension, that is equipped with a $K$-spinor bundle $\mathcal{S}$. Let $\operatorname{det}(\mathcal{S})$ be its determinant bundle, and let $\Phi_{\mathcal{S}}$ be the moment map that is attached to an invariant connection on $\operatorname{det}(\mathcal{S})$. We assume that there exists $(\mathfrak{h}) \in \mathcal{H}_{\mathfrak{k}}$ such that $\left(\left[\mathfrak{k}_{M}, \mathfrak{k}_{M}\right]\right)=([\mathfrak{h}, \mathfrak{h}])$. Let $\mathfrak{z}$ be the center of $\mathfrak{h}$.

We consider an admissible element $\mu \in \mathfrak{z}^{*}$ such that $K_{\mu}=H$ : the coadjoint orbit $\mathcal{P}:=K \mu$ is admissible and contained in the Dixmier sheet $\mathfrak{k}_{(\mathfrak{h})}^{*}$. Let

$$
M_{\mathcal{P}}:=\Phi_{\mathcal{S}}^{-1}(\mathcal{P}) / K
$$

In order to define $\mathrm{Q}^{\text {spin }}\left(M_{\mathcal{P}}\right) \in \mathbb{Z}$, we proceed as follows.

We follow here the notation of $\S 3.3$. Let $C$ be the connected component of

$$
\mathfrak{h}_{0}^{*}:=\left\{\xi \in \mathfrak{h}^{*}: K_{\xi} \subset H\right\}
$$

containing $\mu$. The slice $\mathcal{Y}_{C}=\Phi_{\mathcal{S}}^{-1}(C)$ is an $H$-submanifold of $M$ equipped with an $H$ $\operatorname{spin}^{c}$ bundle $\mathcal{S}_{\mathcal{Y}_{C}}$ : the associated moment map is $\Phi_{\mathcal{Y}_{C}}:=\left.\Phi_{\mathcal{S}}\right|_{\mathcal{Y}_{C}}-\varrho_{C}$, where $\varrho_{C}$ is defined by $(3.15)$.

The element $\tilde{\mu}:=\mu-\varrho(\mu)=\mu-\varrho_{C}$ belongs to the weight lattice $\Lambda$ of the torus $A_{H}:=$ $H /[H, H]$, and the reduced space $M_{K \mu}$ is equal to

$$
\left(\mathcal{Y}_{C}\right)_{\tilde{\mu}}:=\left\{\Phi_{\mathcal{Y}_{C}}=\tilde{\mu}\right\} / A_{H} .
$$

By definition, we take $\mathrm{Q}^{\operatorname{spin}}\left(M_{K \mu}\right):=\mathrm{Q}^{\text {spin }}\left(\left(\mathcal{Y}_{C}\right)_{\tilde{\mu}}\right)$, where the last term is computed as explained in the previous section. More precisely, let us decompose $\mathcal{Y}_{C}$ into its connected components $\mathcal{Y}_{1}, \ldots, \mathcal{Y}_{r}$. For each $j$, let $\mathfrak{z}_{j} \subset \mathfrak{z}$ be the generic infinitesimal stabilizer relative to the $A_{H}$-action on $\mathcal{Y}_{j}$. Then we take

$$
\mathrm{Q}^{\operatorname{spin}}\left(M_{\mathcal{P}}\right)=\mathrm{Q}^{\operatorname{spin}}\left(\left(\mathcal{Y}_{C}\right)_{\tilde{\mu}}\right):=\sum_{j} \mathrm{Q}^{\operatorname{spin}}\left(\left(\mathcal{Y}_{j}\right)_{\tilde{\mu}+\varepsilon_{j}}\right),
$$

where $\varepsilon_{j} \in \mathfrak{z}_{j}^{\perp}$ are generic and small enough.

With this definition of quantization of reduced spaces $\mathrm{Q}^{\operatorname{spin}}\left(M_{\mathcal{P}}\right)$, we obtain the main theorem of this article, inspired by the $[Q, R]=0$ theorem of Meinrenken-Sjamaar.

Let $M$ be a $K$-manifold and $\mathcal{S}$ be a $K$-equivariant $\operatorname{spin}^{c}$-bundle over $M$. Let $(\mathfrak{h}) \in \mathcal{H}_{\mathfrak{k}}$ such that $\left(\left[\mathfrak{k}_{M}, \mathfrak{k}_{M}\right]\right)=([\mathfrak{h}, \mathfrak{h}])$, and consider the set $\mathcal{A}((\mathfrak{h}))$ of admissible orbits contained in the Dixmier sheet $\mathfrak{k}_{(\mathfrak{h})}^{*}$.

THEOREM 5.10.

$$
\mathcal{Q}_{K}(M, \mathcal{S})=\sum_{\mathcal{P} \in \mathcal{A}((\mathfrak{h}))} \mathrm{Q}^{\text {spin }}\left(M_{\mathcal{P}}\right) \mathrm{Q}_{K}^{\text {spin }}(\mathcal{P})
$$


We end this section by giving yet another criterium for the vanishing of $\mathcal{Q}_{K}(M, \mathcal{S})$.

Consider the map $\Phi_{\mathcal{S}}: M \rightarrow \mathfrak{k}^{*}$. At each point $m \in M$, the differential $d_{m} \Phi_{\mathcal{S}}$ defines a linear map $\mathrm{T}_{m} M \rightarrow \mathfrak{k}^{*}$. Let $\mathfrak{k}_{m}^{\perp} \subset \mathfrak{k}^{*}$. From the Kostant relations, we see that $d_{m} \Phi_{\mathcal{S}}$ takes value in $\mathfrak{k}_{m}^{\perp}$.

Proposition 5.11. - Suppose that the group $K$ is abelian and that $\left(\mathfrak{k}_{M}\right)=0$. Then $\mathcal{Q}_{K}(M, \mathcal{S}) \neq 0$ only if the image of $\Phi_{\mathcal{S}}$ contains an element of the weight lattice in its interior.

- For a general group action, we have $\mathcal{Q}_{K}(M, \mathcal{S}) \neq 0$ only if the subset

$$
C_{M}:=\left\{m \in M: \operatorname{Image}\left(d_{m} \Phi_{\mathcal{S}}\right)=\mathfrak{k}_{m}^{\perp}\right\}
$$

has non-empty interior.

Proof. If the group $K$ is abelian, and $\left(\mathfrak{k}_{M}\right)=0$, then the affine subspace $I(M)$ is equal to $\mathfrak{k}^{*}$. The first point follows then from Remark 5.6.

Let us prove the second point. If $\mathcal{Q}_{K}(M, \mathcal{S}) \neq 0$, then $\mathrm{Q}^{\operatorname{spin}}\left(M_{\mathcal{P}}\right) \neq 0$ for an admissible orbit $\mathcal{P}=K \mu$ of type (h). If we consider the decomposition of the slice $\mathcal{Y}_{C}=\bigcup_{j} \mathcal{Y}_{j}$ in connected components, then, for some $j, \mathrm{Q}^{\operatorname{spin}}\left(\left(\mathcal{Y}_{j}\right)_{\tilde{\mu}+\varepsilon_{j}}\right) \neq 0$ (see (5.39)). Thus, due to Remark 5.6, we know that the image of $\Phi_{\mathcal{Y}_{j}}$ has non-empty interior in $\mathfrak{z}_{j}^{\perp}$, and therefore $\left\{y \in \mathcal{Y}_{j}\right.$ :Image $\left.\left(d_{y} \Phi_{\mathcal{Y}_{j}}\right)=\mathfrak{z}_{j}^{\perp}\right\}$ has non-empty interior in $\mathcal{Y}_{j}$. By definition, $\mathfrak{z}_{j}$ is the infinitesimal stabilizer of the action of $H /[H, H]$ on $\mathcal{Y}_{j}$. Thus, $\mathfrak{z}_{j}^{\perp} \subset \mathfrak{z}^{*}$ is equal to $\mathfrak{k}_{y}^{\perp}$ for generic $y \in \mathcal{Y}_{j}$. It follows that $C_{\mathcal{Y}_{j}}:=\left\{y \in \mathcal{Y}_{j}: \operatorname{Image}\left(d_{y} \Phi_{\mathcal{Y}_{j}}\right)=\mathfrak{k}_{y}^{\perp}\right\}$ has non-empty interior in $\mathcal{Y}_{j}$. Finally, the set $K C_{\mathcal{Y}_{j}} \subset C_{M}$ has non-empty interior in $M$.

Corollary 5.12. If the $K$-action is non-trivial, we have $\mathcal{Q}_{K}(M, \mathcal{S})=0$ if

(a) $\Phi_{\mathcal{S}}$ is a constant map, or

(b) the 2-form $\Omega_{\mathcal{S}}$ is exact.

Proof. If the $K$-action is non-trivial and $\Phi_{\mathcal{S}}$ is constant, the set $C_{M}=M^{K}$ is a closed submanifold of $M$ with empty interior. Due to Proposition 5.11, we must have $\mathcal{Q}_{K}(M, \mathcal{S})=0$.

If $\Omega_{\mathcal{S}}=d \alpha$, by modifying the connection on $\operatorname{det}(\mathcal{S})$ by $\alpha$, our moment map is constant, and we conclude that $\mathcal{Q}_{K}(M, \mathcal{S})=0$ by the previous case.

Note that Corollary 5.12 implies the well-known Atiyah-Hirzebruch vanishing theorem in the spin case [3], as well as the variant of Hattori [15].

\section{Examples: multiplicities and reduced spaces}

In this last subsection, we give some simple examples in order to illustrate various features of our result relating multiplicities and reduced spaces. An open question remains even 
for a toric manifold $M$ equipped with a non ample line bundle $L$. The determinant line bundle of the spinor bundle $\mathcal{S}:=\bigwedge_{\mathbb{C}} \mathrm{T} M \otimes L$ is equal to $\operatorname{det}(\mathcal{S}):=\operatorname{det}_{\mathbb{C}}(\mathrm{T} M) \otimes L^{\otimes 2}$. A connection $\nabla$ on $\operatorname{det}(\mathcal{S})$ determines a moment map $\Phi_{\nabla}: M \rightarrow \mathfrak{t}^{*}$ and a curvature

$$
\Omega_{\nabla}=\frac{1}{2 i} \nabla^{2}
$$

The push-forward of $\Omega_{\nabla}$ by $\Phi_{\nabla}$ does not depend on the choice of the connection: it is a signed measure, denoted by $\operatorname{DH}(M, \mathcal{S})$, and called the Duistermaat-Heckmann measure. The support of $\operatorname{DH}(M, \mathcal{S})$, which is contained in $\Phi_{\nabla}(M)$, is a union of convex polytopes. Can we find $\nabla$ such that the image $\Phi_{\nabla}(M)$ is exactly the support of the Duistermaat-Heckman measure?

\subsection{The reduced space might not be connected}

We consider the simplest case of the theory. Let $\mathbb{P}^{1}:=\mathbb{P}^{1}(\mathbb{C})$ be the projective space of (complex) dimension 1. Consider the (ample) line bundle $\mathcal{L} \rightarrow \mathbb{P}^{1}$, dual of the tautological bundle. It is obtained as quotient of the trivial line bundle $\mathbb{C}^{2} \backslash\{(0,0)\} \times \mathbb{C}$ on $\mathbb{C}^{2} \backslash\{(0,0)\}$ by the action $u \cdot\left(z_{1}, z_{2}, z\right)=\left(u z_{1}, u z_{2}, u z\right)$ of $\mathbb{C}^{*}$. We consider the action of $T=S^{1}$ on $\mathcal{L} \rightarrow \mathbb{P}^{1}$ defined by $t \cdot\left[z_{1}, z_{2}, z\right]=\left[t^{-1} z_{1}, z_{2}, z\right]$.

Let $\mathcal{S}(n)$ be the $\operatorname{spin}^{c}$-bundle $\bigwedge_{\mathbb{C}} \mathrm{TP}^{1} \otimes \mathcal{L}^{\otimes n}$. The character $\mathcal{Q}_{T}(M, \mathcal{S}(n))$ is equal to $H^{0}\left(\mathbb{P}^{1}, \mathcal{O}(n)\right)-H^{1}\left(\mathbb{P}^{1}, \mathcal{O}(n)\right)$, where $\mathcal{O}(n)$ is the sheaf of holomorphic sections of $\mathcal{L}^{\otimes n}$. Note that the holomorphic line bundle $\mathcal{L}^{\otimes n}$ is not ample if $n \leqslant 0$. We have

- $\mathcal{Q}_{T}(M, \mathcal{S}(n))=-\sum_{k=n+1}^{-1} t^{k}$ when $n \leqslant-2$,

- $\mathcal{Q}_{T}(M, \mathcal{S}(-1))=0$,

- $\mathcal{Q}_{T}(M, \mathcal{S}(n))=\sum_{k=0}^{n} t^{k}$ when $n \geqslant 0$.

The determinant line bundle of $\mathcal{S}(n)$ is $\mathbb{L}_{n}=\left[\mathbb{C}_{-1}\right] \otimes \mathcal{L}^{\otimes 2 n+2}$, where $\left[\mathbb{C}_{-1}\right]$ is the trivial line bundle equipped with the representation $t^{-1}$ on $\mathbb{C}$.

Remark that $\mathbb{P}^{1}$ is homogeneous under $U(2)$, so there exists a unique $U(2)$-invariant connection on $\mathbb{L}_{n}$. The corresponding moment map $\Phi_{\mathcal{S}(n)}$ is such that

$$
\Phi_{\mathcal{S}(n)}\left(\left[z_{1}, z_{2}\right]\right)=(n+1) \frac{\left|z_{1}\right|^{2}}{\left|z_{1}\right|^{2}+\left|z_{2}\right|^{2}}-\frac{1}{2} .
$$

The image $I_{n}=\Phi_{\mathcal{S}(n)}(M)$ is

- the interval $\left[-\frac{1}{2}, n+\frac{1}{2}\right]$ when $n \geqslant 0$,

- a point $\left\{-\frac{1}{2}\right\}$ when $n=-1$,

- the interval $\left[n+\frac{1}{2},-\frac{1}{2}\right]$ when $n \leqslant-2$.

It is in agreement with our theorem. Indeed all characters occurring in $\mathcal{Q}_{T}(M, \mathcal{S}(n))$ are the integral points in the relative interior of $I_{n}$, and all reduced spaces are points. 


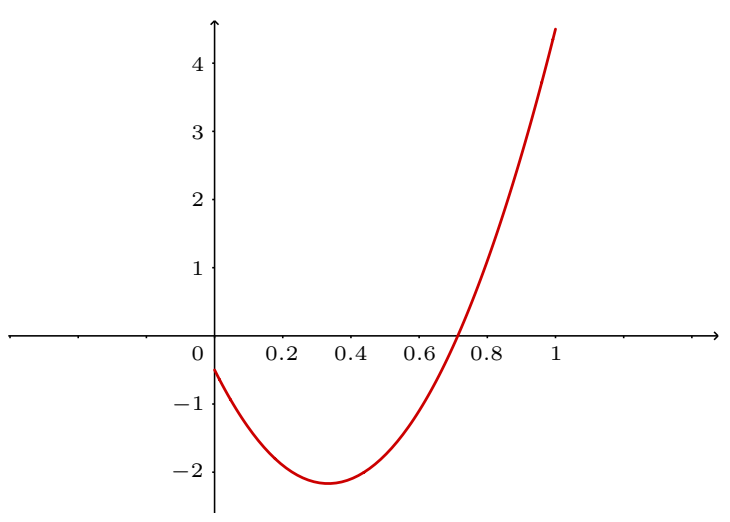

Figure 8. The graph of $\Phi$.

If we consider simply the action of $T$ on $\mathbb{P}^{1}$, the choice of connection may vary. In fact, given any smooth function $f$ on $\mathbb{R}$, we can define a connection $\nabla^{f}$ on $\mathbb{L}_{n}$ such that the corresponding moment map is

$$
\Phi_{\mathcal{S}(n)}^{f}\left(\left[z_{1}, z_{2}\right]\right)=\Phi_{\mathcal{S}(n)}\left(\left[z_{1}, z_{2}\right]\right)+f\left(\frac{\left|z_{1}\right|^{2}}{\left|z_{1}\right|^{2}+\left|z_{2}\right|^{2}}\right) \frac{\left|z_{1}\right|^{2}\left|z_{2}\right|^{2}}{\left(\left|z_{1}\right|^{2}+\left|z_{2}\right|^{2}\right)^{2}}
$$

Let $\Omega_{\mathcal{S}(n)}^{f}$ be one half of the curvature of $\left(\mathbb{L}_{n}, \nabla^{f}\right)$; then the Duistermaat-Heckman measure $\left(\Phi_{\mathcal{S}(n)}^{f}\right)_{*} \Omega_{\mathcal{S}(n)}^{f}$ is independent of the choice of the connection $\nabla^{f}$ and is equal to the characteristic function of $I_{n}$.

Take, for example, $n=4$ and the constant function $f(x)=-15$. the corresponding moment map is

$$
\Phi\left(\left[z_{1}, z_{2}\right]\right)=5 \frac{\left|z_{1}\right|^{2}}{\left|z_{1}\right|^{2}+\left|z_{2}\right|^{2}}-15 \frac{\left|z_{1}\right|^{2}\left|z_{2}\right|^{2}}{\left(\left|z_{1}\right|^{2}+\left|z_{2}\right|^{2}\right)^{2}}-\frac{1}{2}
$$

Figure 8 is the graph of $\Phi$ in terms of

$$
x=\frac{\left|z_{1}\right|^{2}}{\left|z_{1}\right|^{2}+\left|z_{2}\right|^{2}}
$$

varying between 0 and 1 . We see that the image of $\Phi$ is the interval $\left[-\frac{13}{6}, \frac{9}{2}\right]$, but the image of the signed measure is still $\left[-\frac{1}{2}, \frac{9}{2}\right]$ : so for this choice of connection the image of $\Phi$ is larger than the support of the Duistermaat-Heckman measure.

Above the integral points in $\left[-\frac{13}{6},-\frac{1}{2}\right]$, the reduced space is not connected, it consists of two points giving opposite contributions to the index. So our theorem holds. 


\subsection{The image of the moment map might be non-convex}

Let $M$ be the Hirzebruch surface. Represent $M$ as the quotient of $\mathcal{U}=\left(\mathbb{C}^{2} \backslash\{(0,0)\}\right) \times$ $\left(\mathbb{C}^{2} \backslash\{(0,0)\}\right)$ by the free action of $\mathbb{C}^{*} \times \mathbb{C}^{*}$ acting by

$$
(u, v) \cdot\left(z_{1}, z_{2}, z_{3}, z_{4}\right)=\left(u z_{1}, u z_{2}, u v z_{3}, v z_{4}\right),
$$

and we denote by $\left[z_{1}, z_{2}, z_{3}, z_{4}\right] \in M$ the equivalence class of $\left(z_{1}, z_{2}, z_{3}, z_{4}\right)$. The map $\pi:\left[z_{1}, z_{2}, z_{3}, z_{4}\right] \mapsto\left[z_{1}, z_{2}\right]$ is a fibration of $M$ on $P_{1}(\mathbb{C})$ with fiber $P_{1}(\mathbb{C})$.

Consider the line bundle $L\left(n_{1}, n_{2}\right)$ obtained as quotient of the trivial line bundle $\mathcal{U} \times \mathbb{C}$ on $\mathcal{U}$ by the action

$$
(u, v) \cdot\left(z_{1}, z_{2}, z_{3}, z_{4}, z\right)=\left(u z_{1}, u z_{2}, u v z_{3}, v z_{4}, u^{n_{1}} v^{n_{2}} z\right)
$$

for $(u, v) \in \mathbb{C}^{*} \times \mathbb{C}^{*}$. The line bundle $L\left(n_{1}, n_{2}\right)$ is ample if and only if $n_{1}>n_{2}>0$.

We have a canonical action of the group $K:=U(2)$ on $M: g \cdot\left[Z_{1}, Z_{2}\right]=\left[g Z_{1}, Z_{2}\right]$ for $Z_{1}, Z_{2} \in \mathbb{C}^{2} \backslash\{(0,0)\}$ and the line bundle $L\left(n_{1}, n_{2}\right)$ with action $g \cdot\left[Z_{1}, Z_{2}, z\right]=\left[g Z_{1}, Z_{2}, z\right]$ is $K$-equivariant.

We are interested in the (virtual) $K$-module

$$
H^{0}\left(M, \mathcal{O}\left(n_{1}, n_{2}\right)\right)-H^{1}\left(M, \mathcal{O}\left(n_{1}, n_{2}\right)\right)+H^{2}\left(M, \mathcal{O}\left(n_{1}, n_{2}\right)\right),
$$

where $\mathcal{O}\left(n_{1}, n_{2}\right)$ be the sheaf of holomorphic sections of $L\left(n_{1}, n_{2}\right)$.

In this case, it is in fact possible to compute directly individual cohomology groups $H^{i}\left(M, \mathcal{O}\left(n_{1}, n_{2}\right)\right)$. However, we will describe here only results on the alternate sum and relate them to the moment map.

Let $T=U(1) \times U(1)$ be the maximal torus of $K$. The set

$$
Y:=\left\{\left[z_{1}, z_{2}, z_{3}, z_{4}\right] \in M: z_{1}=0\right\}
$$

is a $T$-invariant complex submanifold of $M$ (with trivial action of $\left(t_{1}, 1\right)$ ). The map

$$
\begin{aligned}
Y & \longrightarrow \mathbb{P}^{1}(\mathbb{C}), \\
{\left[0, z_{2}, z_{3}, z_{4}\right] } & \longmapsto\left[z_{2}^{-1} z_{3}, z_{4}\right],
\end{aligned}
$$

is a $T$-equivariant isomorphism and the map

$$
\begin{gathered}
K \times Y \longrightarrow M, \\
(g, y) \longmapsto g \cdot y,
\end{gathered}
$$

factorizes through an isomorphism $K \times_{T} Y \simeq M$. Thus $M$ is an induced manifold. 
For any $(a, b) \in \mathbb{Z}^{2}$, we denote by $\mathbb{C}_{a, b}$ the 1 -dimensional representation of $T$ associated with the character $\left(t_{1}, t_{2}\right) \mapsto t_{1}^{a} t_{2}^{b}$. We denote by $\left\{e_{1}^{*}, e_{2}^{*}\right\}$ the canonical basis of $\mathfrak{t}^{*} \simeq \mathbb{R}^{2}$. The Weyl chamber is $\mathfrak{t}_{\geqslant 0}^{*}=\left\{x e_{1}^{*}+y e_{2}^{*}: x \geqslant y\right\}$. The elements $e_{1}^{*}$ and $e_{2}^{*}$ are conjugated by the Weyl group.

Note that the line bundle $L\left(n_{1}, n_{2}\right)$, when restricted to $Y \simeq \mathbb{P}^{1}(\mathbb{C})$, is isomorphic to $\mathcal{L}^{\otimes n_{2}} \otimes\left[\mathbb{C}_{0,-n_{1}}\right]$.

We consider the line bundle $L_{\varkappa}=L(3,2)$ obtained from the reduction of the trivial line bundle $\bigwedge^{4} \mathbb{C}^{4}$ with natural action of $\mathbb{C}^{*} \times \mathbb{C}^{*}$. We denote $\mathcal{S}_{M}:=\bigwedge_{\mathbb{C}} \mathrm{T} M$ (resp. $\mathcal{S}_{Y}:=$ $\left.\bigwedge_{\mathbb{C}} \mathrm{T} Y\right)$ the $\operatorname{spin}^{c}$-bundle associated with the complex structure on $M$ (resp. $Y$ ).

We denote by $\varphi: Y \rightarrow[0,1]$ the map defined by

$$
\varphi(y)=\frac{\left|a_{1}\right|^{2}}{\left|a_{1}\right|^{2}+\left|a_{2}\right|^{2}} \quad \text { if } y \simeq\left[a_{1}, a_{2}\right] .
$$

Proposition 6.1. - Let $\mathcal{S}\left(n_{1}, n_{2}\right)$ be the spin bundle $\mathcal{S}_{M} \otimes L\left(n_{1}, n_{2}\right)$ on $M$. Its determinant line bundle is

$$
\mathbb{L}_{n_{1}, n_{2}}=\left[\mathbb{C}_{\text {det }}\right] \otimes L_{\varkappa} \otimes L\left(2 n_{1}, 2 n_{2}\right),
$$

where $\left[\mathbb{C}_{\mathrm{det}}\right] \rightarrow M$ is the trivial $U(2)$-equivariant line bundle associated with the character $\operatorname{det}: U(2) \rightarrow \mathbb{C}^{*}$.

- There exists a connection on $\mathbb{L}_{n_{1}, n_{2}}$ such that the corresponding moment map $\Phi_{n_{1}, n_{2}}: K \times_{T} Y \rightarrow \mathfrak{k}^{*}$ is defined by

$$
\Phi_{n_{1}, n_{2}}([k, y])=\left(-\left(n_{1}+\frac{3}{2}\right)+\left(n_{2}+1\right) \varphi(y)\right) k \cdot e_{2}^{*}+\frac{1}{2}\left(e_{1}^{*}+e_{2}^{*}\right)
$$

Proof. For the second point, we construct a $U(2)$-invariant connection on $\mathbb{L}_{n_{1}, n_{2}}$ by choosing the $T$-invariant connection on $\left.\left(\mathbb{L}_{n_{1}, n_{2}}\right)\right|_{Y}$ having moment map

$$
\left(-\left(n_{1}+\frac{3}{2}\right)+\left(n_{2}+1\right) \varphi(y)\right) e_{2}^{*}+\frac{1}{2}\left(e_{1}^{*}+e_{2}^{*}\right)
$$

under the $T$-action (see equation (6.41)).

From Proposition 6.1, it is not difficult to describe the "Kirwan set"

$$
\Delta\left(n_{1}, n_{2}\right)=\operatorname{Image}\left(\Phi_{n_{1}, n_{2}}\right) \cap \mathfrak{t}_{\geqslant 0}^{*}
$$

for all cases of $n_{1}$ and $n_{2}$. It depends of the signs of $n_{1}+\frac{3}{2}, n_{2}+1, n_{1}-n_{2}+\frac{1}{2}$, that is, as we are working with integers, the signs of $n_{1}+1, n_{2}+1$ and $n_{1}-n_{2}$. We concentrate 
in the case where $n_{1}+1 \geqslant 0$ and $n_{2}+1 \geqslant 0$ (other cases are similarly treated). Then, we have two cases:

- If $n_{1} \geqslant n_{2}$, then the Kirwan set $\Delta\left(n_{1}, n_{2}\right)$ is the interval

$$
\left[\left(n_{1}-n_{2}\right)+\frac{1}{2}, n_{1}+\frac{3}{2}\right]\left(-e_{2}^{*}\right)+\frac{1}{2}\left(e_{1}^{*}+e_{2}^{*}\right) .
$$

- If $n_{2}>n_{1}$, then the Kirwan set $\Delta\left(n_{1}, n_{2}\right)$ is the union of the intervals

$$
\left[0, n_{2}-n_{1}-\frac{1}{2}\right] e_{1}^{*}+\frac{1}{2}\left(e_{1}^{*}+e_{2}^{*}\right)
$$

and

$$
\left[0, n_{1}+\frac{3}{2}\right]\left(-e_{2}^{*}\right)+\frac{1}{2}\left(e_{1}^{*}+e_{2}^{*}\right)
$$

If $n_{1} \geqslant n_{2} \geqslant 0$, the curvature of the corresponding connection on

$$
\mathbb{L}_{n_{1}, n_{2}}=L\left(2 n_{1}+3,2 n_{2}+2\right)
$$

(which is an ample line bundle) is non-degenerate, thus the image is a convex subset of $\mathfrak{t}_{\geqslant 0}^{*}$ (in agreement with Kirwan convexity theorem), while for $n_{2}>n_{1}$ the image set is not convex.

The character $\mathcal{Q}_{K}\left(n_{1}, n_{2}\right):=\mathcal{Q}_{K}\left(M, \mathcal{S}\left(n_{1}, n_{2}\right)\right)$ is equal to the (virtual) $K$-module $H^{0}\left(M, \mathcal{O}\left(n_{1}, n_{2}\right)\right)-H^{1}\left(M, \mathcal{O}\left(n_{1}, n_{2}\right)\right)+H^{2}\left(M, \mathcal{O}\left(n_{1}, n_{2}\right)\right)$, where $\mathcal{O}\left(n_{1}, n_{2}\right)$ is the sheaf of holomorphic sections of $L\left(n_{1}, n_{2}\right)$.

Let $\Lambda_{\geqslant 0}=\left\{\left(\lambda_{1}, \lambda_{2}\right) ; \lambda_{1} \geqslant \lambda_{2}\right\}$ be the set of dominant weights for $U(2)$. We index the representations of $U(2)$ by $\varrho+\Lambda_{\geqslant 0}$. Here $\varrho=\left(\frac{1}{2},-\frac{1}{2}\right)$, and $\lambda_{1}$ and $\lambda_{2}$ are integers. We then have

$$
\pi_{(1 / 2,-k-1 / 2)}=S^{k}\left(\mathbb{C}^{2}\right)
$$

where $S^{k}\left(\mathbb{C}^{2}\right)$ is the space of complex polynomials on $\mathbb{C}^{2}$ homogeneous of degree $k$.

If $n_{2} \geqslant 0$, we know that $\mathcal{Q}_{T}\left(Y, \mathcal{S}_{Y} \otimes \mathcal{L}^{\otimes n_{2}}\right)=\sum_{k=0}^{n_{2}} t_{2}^{k}$. From the induction formula (3.17) (or direct computation via Cech cohomology!) we obtain the following:

- If $n_{1} \geqslant n_{2}$, then

$$
\mathcal{Q}_{K}\left(n_{1}, n_{2}\right)=\sum_{k=n_{1}-n_{2}}^{n_{1}} \pi_{(1 / 2,-k-1 / 2)}
$$

- If $n_{2}>n_{1}$, then

$$
\mathcal{Q}_{K}\left(n_{1}, n_{2}\right)=\sum_{k=0}^{n_{1}} \pi_{(1 / 2,-k-1 / 2)}-\sum_{k=0}^{n_{2}-n_{1}-2} \pi_{(k+3 / 2,1 / 2)} .
$$




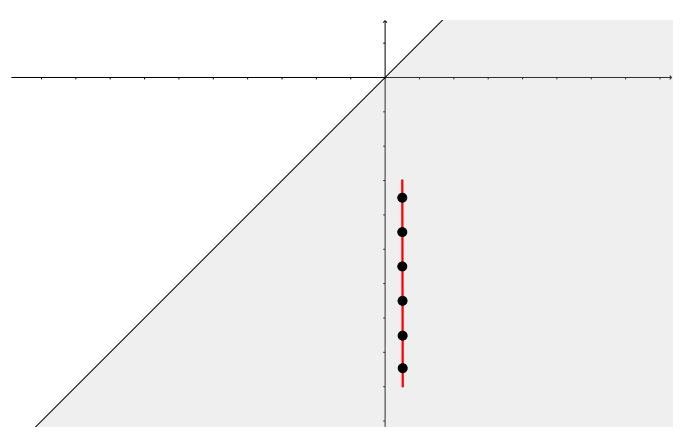

Figure 9. $K$-Multiplicities for $\mathcal{Q}_{K}(8,5)$.

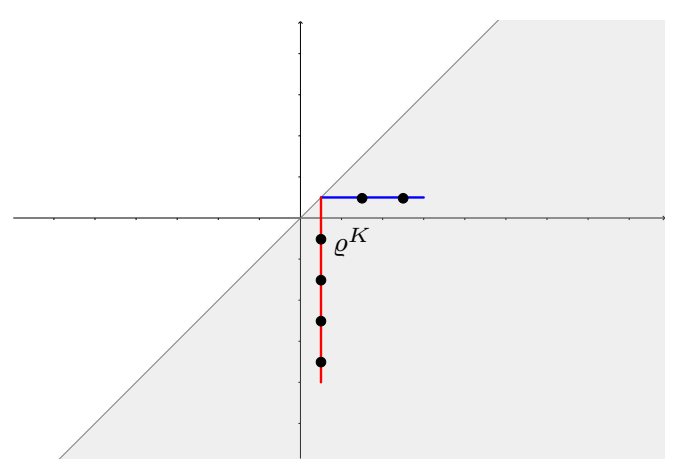

Figure 10. $K$-multiplicities for $\mathcal{Q}_{K}(3,6)$.

Let us check how our theorem works in these cases. First, we notice that we are in a multiplicity free case: all the non-empty reduced spaces are points.

- Consider the case where $n_{1} \geqslant n_{2}$. We see that the parameter $\left(\frac{1}{2},-k-\frac{1}{2}\right)$ belongs to the relative interior of the interval $\Delta\left(n_{1}, n_{2}\right)$ if and only if $n_{1}-n_{2} \leqslant k \leqslant n_{1}$.

- Consider the case where $n_{2}>n_{1}$. We see that the parameter $\left(\frac{1}{2},-k-\frac{1}{2}\right)$ belongs to the relative interior of $\left[-n_{1}-\frac{3}{2}, 0\right] e_{2}^{*}+\frac{1}{2}\left(e_{1}^{*}+e_{2}^{*}\right)$ if and only if $k \leqslant n_{1}$. Similarly, the parameter $\left(k+\frac{3}{2}, \frac{1}{2}\right)$ belongs to the relative interior of $\left[0, n_{2}-n_{1}-\frac{1}{2}\right] e_{1}^{*}+\frac{1}{2}\left(e_{1}^{*}+e_{2}^{*}\right)$ if and only if $k \leqslant n_{2}-n_{1}-2$.

In Figures 9 and 10, we draw the Kirwan subsets of $\mathfrak{t}_{\geqslant 0}^{*}$ corresponding to the values $\left(n_{1}, n_{2}\right)=(8,5)$ and $\left(n_{1}, n_{2}\right)=(3,6)$. The points on the red line represents the admissible points occurring with multiplicity 1 in $\mathcal{Q}_{K}\left(n_{1}, n_{2}\right)$. The points on the blue line represents the admissible points occurring with multiplicity -1 in $\mathcal{Q}_{K}\left(n_{1}, n_{2}\right)$.

Consider now $M$ as a $T$-manifold. Let $\Phi_{n_{1}, n_{2}}^{T}: M \rightarrow \mathfrak{t}^{*}$ be the moment map relative 


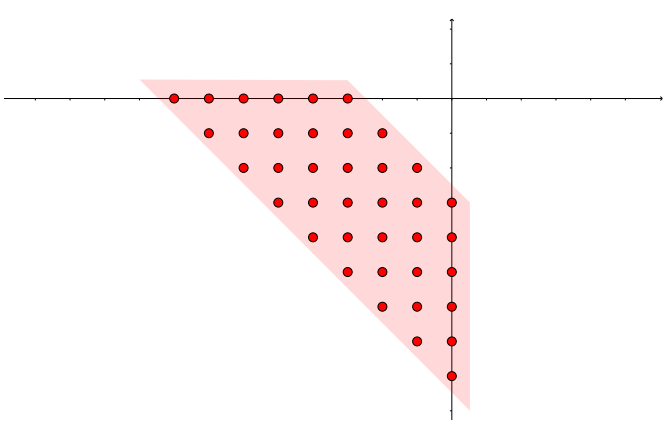

Figure 11. $T$-multiplicities for $\mathcal{Q}_{T}(8,5)$.

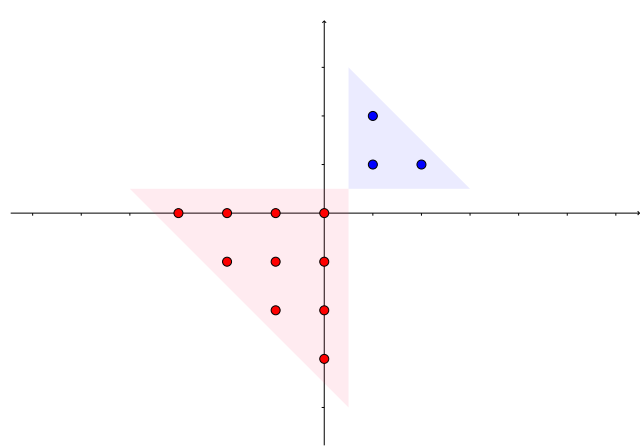

Figure 12. $T$-multiplicities for $\mathcal{Q}_{T}(3,6)$.

to the action of $T$ which is the composition of $\Phi_{n_{1}, n_{2}}: M \rightarrow \mathfrak{k}^{*}$ with the projection $\mathfrak{k}^{*} \rightarrow \mathfrak{t}^{*}$. Thus, the image of $\Phi_{n_{1}, n_{2}}^{T}$ is the convex hull of $\Delta\left(n_{1}, n_{2}\right)$ and its symmetric image with respect to the diagonal.

Consider first the case where $\left(n_{1}, n_{2}\right)=(8,5)$. Thus our determinant bundle $\mathbb{L}_{8,5}$ is ample. The image of the moment map $\Phi_{8,5}^{T}: M \rightarrow \mathfrak{t}^{*}$ is drawn in Figure 11. It is a convex polytope with vertices $\left(-3, \frac{1}{2}\right),\left(\frac{1}{2},-3\right),\left(\frac{1}{2},-9\right)$ and $\left(-9, \frac{1}{2}\right)$, the images of the four fixed points $[1,0,1,0],[1,0,0,1],[0,1,1,0]$ and $[0,1,0,1]$.

We now concentrate on the case $\left(n_{1}, n_{2}\right)=(3,6)$. The line bundle $\mathbb{L}:=\mathbb{L}_{3,6}$ is not ample, so that its curvature $\Omega_{\mathbb{L}}$ is degenerate, and the Liouville form $\beta_{\mathbb{L}}=\Omega_{\mathbb{L}} \wedge \Omega_{\mathbb{L}}$ is a signed measure on $M$. Let us draw the Duistermaat-Heckman measure $\left(\Phi_{\mathbb{L}}\right)_{*} \beta_{\mathbb{L}}$, a signed measure on $\mathfrak{t}^{*}$. In red the measure is with value 1 , in blue the measure is with value -1 (see Figure 12). 
We also verify that our theorem is true. Indeed, the representation $\mathcal{Q}_{T}(3,6)$ is

$$
1+t_{1}^{-1}+t_{2}^{-1}+t_{1}^{-2}+t_{1}^{-1} t_{2}^{-1}+t_{2}^{-2}+t_{1}^{-3}+t_{1}^{-2} t_{2}^{-1}+t_{1}^{-1} t_{1}^{-2}+t_{2}^{-3}-t_{1} t_{2}-t_{1} t_{2}^{2}-t_{1}^{2} t_{2} .
$$

The $\lambda \in \mathbb{Z}^{2}$ such that $t^{\lambda}$ occurs in $\mathcal{Q}_{T}(3,6)$ are the integral points in the interior of the image of $\Phi_{\mathbb{L}}(M)$ : they have multiplicity \pm 1 , and the reduced spaces are points.

In this case, we verify thus that the image of the moment map is exactly the support of the Duitermaat-Heckman measure, however, we do not know if (even in the toric case, and non-ample lines bundles) we can always find a connection with this property.

\subsection{The multiplicity of the trivial representation comes from two reduced spaces}

Consider $\mathbb{C}^{4}$ with its canonical basis $\left\{e_{1}, \ldots, e_{4}\right\}$. Let $K \simeq \mathrm{SU}(3)$ be the subgroup of $\mathrm{SU}(4)$ that fixes $e_{4}$.

Let $T=S(U(1) \times U(1) \times U(1))$ be the maximal torus of $K$ with Lie algebra

$$
\mathfrak{t}=\left\{\left(x_{1}, x_{2}, x_{3}\right): x_{1}+x_{2}+x_{3}=0\right\}
$$

and Weyl chamber $\mathfrak{t}_{\geqslant 0}^{*}:=\left\{\xi_{1} \geqslant \xi_{2} \geqslant \xi_{3}: \xi_{1}+\xi_{2}+\xi_{3}=0\right\}$. We choose the fundamental roots $\omega_{1}$ and $\omega_{2}$ so that $K_{\omega_{1}}=S(U(2) \times U(1))$ and $K_{\omega_{2}}=S(U(1) \times U(2))$. Recall that $\omega_{1}, \omega_{2}$ generates the weight lattice $\Lambda \subset \mathfrak{t}^{*}$ so that $\Lambda_{\geqslant 0}=\mathbb{N} \omega_{1}+\mathbb{N} \omega_{2}$. Also note that $\varrho=\omega_{1}+\omega_{2}$. For any $\lambda \in \Lambda_{\geqslant 0}+\varrho$, we denote by $\pi_{\lambda}$ the irreducible representation of $K$ with highest weight $\lambda-\varrho$.

Let $X=\left\{0 \subset L_{1} \subset L_{2} \subset \mathbb{C}^{4}: \operatorname{dim} L_{i}=i\right\}$ be the homogeneous partial flag manifold under the action of $\mathrm{SU}(4)$. We have two lines bundles over $X: \mathcal{L}_{1}(x)=L_{1}$ and $\mathcal{L}_{2}(x)=L_{2} / L_{1}$ for $x=\left(L_{1}, L_{2}\right)$.

Our object of study is the complex submanifold

$$
M=\left\{\left(L_{1}, L_{2}\right) \in X: \mathbb{C} e_{4} \subset L_{2}\right\}
$$

The group $K$ acts on $M$, and the generic stabilizer of the action is $\left[K_{\omega_{1}}, K_{\omega_{1}}\right] \simeq \mathrm{SU}(2)$. We consider the family of lines bundles

$$
\mathcal{L}(a, b)=\left.\left.\mathcal{L}_{1}^{\otimes a}\right|_{M} \otimes \mathcal{L}_{2}^{\otimes-b}\right|_{M}, \quad(a, b) \in \mathbb{N}^{2} .
$$

Let $\mathcal{S}_{M}:=\bigwedge_{\mathbb{C}} \mathrm{T} M$ be the $\operatorname{spin}^{c}$-bundle associated with the complex structure on $M$. We compute the characters

$$
\mathcal{Q}_{K}(a, b):=\mathcal{Q}_{K}\left(M, \mathcal{S}_{M} \otimes \mathcal{L}(a, b)\right) \in R(K)
$$


Again,

$$
\mathcal{Q}_{K}(a, b)=\sum_{i=0}^{\operatorname{dim} M}(-1)^{i} H^{i}(M, \mathcal{O}(\mathcal{L}(a, b))) .
$$

We notice that $K_{\omega_{1}}$ corresponds to the subgroup of $K$ that fixes the line $\mathbb{C} e_{3}$. The set $Y:=\left\{\left(L_{1}, L_{2}\right) \in X: L_{2}=\mathbb{C} e_{3} \oplus \mathbb{C} e_{4}\right\}$ is a $K_{\omega_{1}}$-invariant complex submanifold of $M$ such that the map $K \times Y \ni(k, y) \mapsto k y \in M$ factorizes through an isomorphism $K \times_{K_{\omega_{1}}} Y \simeq M$. Notice that $\left[K_{\omega_{1}}, K_{\omega_{1}}\right]$ acts trivially on $Y$.

If we take $a \geqslant 4$ and $b \geqslant 1$, we get that

$$
\mathcal{Q}_{K}(a, b)=-\sum_{k=0}^{b-1} \pi_{k \omega_{1}+\varrho}-\sum_{j=0}^{a-4} \pi_{j \omega_{2}+\varrho} .
$$

In particular, the multiplicity of $\pi_{\varrho}$ (the trivial representation) in $\mathcal{Q}_{K}(a, b)$ is equal to -2 .

We now verify the formula (5.40) in our case. The $\operatorname{spin}^{c}$-bundle $\mathcal{S}_{M}$ is equal to $\mathcal{S}_{K \omega_{1}} \otimes K \times_{K_{\omega_{1}}} \mathcal{S}_{Y}$. The corresponding determinant line bundle $\operatorname{det}\left(\mathcal{S}_{M}\right)$ satisfies

$$
\operatorname{det}\left(\mathcal{S}_{M}\right)=K \times_{K_{\omega_{1}}} \mathbb{C}_{3 \omega_{1}} \otimes K \times_{K_{\omega_{1}}} \operatorname{det}\left(\mathcal{S}_{Y}\right)=K \times_{K_{\omega_{1}}} \mathbb{C}_{2 \omega_{1}} \otimes \mathcal{L}_{1}^{\otimes-2}
$$

Hence, for the $\operatorname{spin}^{c}$-bundle $\mathcal{S}_{M} \otimes \mathcal{L}(a, b)$, we have

$$
\operatorname{det}\left(\mathcal{S}_{M} \otimes \mathcal{L}(a, b)\right)=\operatorname{det}\left(\mathcal{S}_{M}\right) \otimes \mathcal{L}(a, b)^{\otimes 2}=K \times_{K_{\omega_{1}}} \mathbb{C}_{(2 b+2) \omega_{1}} \otimes \mathcal{L}_{1}^{\otimes 2(a+b-1)}
$$

The line bundle $\operatorname{det}\left(\mathcal{S}_{M} \otimes \mathcal{L}(a, b)\right)$ is equipped with a natural holomorphic and Hermitian connection $\nabla$. To compute the corresponding moment map $\Phi_{a, b}: M \rightarrow \mathfrak{k}^{*}$, we notice that $\mathcal{L}_{1}=K \times_{K_{\omega_{1}}} \mathcal{L}^{-1}$, where $\mathcal{L} \rightarrow \mathbb{P}^{1}$ is the prequantum line bundle over $\mathbb{P}_{1}$ (equipped with the Fubini-Study symplectic form). If we let $\varphi: Y \simeq \mathbb{P}^{1} \rightarrow[0,1]$ be the function defined by

$$
\varphi\left(\left[z_{1}, z_{2}\right]\right)=\frac{\left|z_{1}\right|^{2}}{\left|z_{1}\right|^{2}+\left|z_{2}\right|^{2}},
$$

we see that

$$
\Phi_{a, b}([k, y])=k\left[((b+1)-(a+b-1) \varphi(y)) \omega_{1}\right]
$$

for $[k, y] \in M$. In this case, the Kirwan set $\Phi_{a, b}(M) \cap \mathfrak{t}_{\geqslant 0}^{*}$ is the non-convex set

$$
[0, b+1] \omega_{1} \cup[0, a-2] \omega_{2}
$$

We know (see Exemple 3.9) that the set $\mathcal{A}\left(\left(\mathfrak{k}_{\omega_{1}}\right)\right)$ is equal to the collection of orbits $K\left(\frac{1}{2}(1+2 n) \omega_{i}\right), n \in \mathbb{N}, i=1,2$, and we have $\mathcal{Q}_{K}\left(K\left(\frac{1}{2} \omega_{i}\right)\right)=0$ and $\mathcal{Q}_{K}\left(K\left(\frac{1}{2}(3+2 k) \omega_{i}\right)\right)=$ $\pi_{k \omega_{i}+\varrho}$ when $k \geqslant 0$. 
If we apply (5.40), we see that $\pi_{k \omega_{1}+\varrho}$ occurs in $\mathcal{Q}_{K}(a, b)$ only if $\frac{1}{2}(3+2 k)<b+1$ : so $k \in\{0, \ldots, b-1\}$. Similarly $\pi_{j \omega_{2}+\varrho}$ occurs in $\mathcal{Q}_{K}(a, b)$ only if $\frac{1}{2}(3+2 j)<a-2$ : thus $j \in\{0, \ldots, a-4\}$. For all these cases the corresponding reduced spaces are points and one could check that the corresponding quantizations are all equal to -1 (see (5.36)).

In these cases, two orbits $\mathcal{P}_{i}=K\left(\frac{3}{2} \omega_{i}\right), i=1,2$, are the ancestors of the trivial representation in $\mathcal{A}\left(\left(\mathfrak{k}_{\omega_{1}}\right)\right)$, and the multiplicity of the trivial representation in

$$
\mathcal{Q}_{K}\left(M, \mathcal{S}_{M} \otimes \mathcal{L}(a, b)\right)
$$

is equal to

$$
\mathrm{Q}^{\mathrm{spin}}\left(M_{\mathcal{P}_{1}}\right)+\mathrm{Q}^{\mathrm{spin}}\left(M_{\mathcal{P}_{2}}\right)=-2 .
$$

\section{References}

[1] АтіуAн, M.F., Elliptic Operators and Compact Groups. Lecture Notes in Mathematics, 401. Springer, Berlin-New York, 1974.

[2] Атіуан, M.F. \& Botт, R., The Yang-Mills equations over Riemann surfaces. Philos. Trans. Roy. Soc. London Ser. A, 308 (1983), 523-615.

[3] Atiyah, M. F. \& Hirzebruch, F., Spin-manifolds and group actions, in Essays on Topology and Related Topics (Mémoires dédiés à Georges de Rham), pp. 18-28. Springer, New York, 1970.

[4] Berline, N., Getzler, E. \& Vergne, M., Heat Kernels and Dirac Operators. Grundlehren Text Editions. Springer, Berlin-Heidelberg, 2004.

[5] Berline, N. \& Vergne, M., L'indice équivariant des opérateurs transversalement elliptiques. Invent. Math., 124 (1996), 51-101.

[6] Braverman, M., Index theorem for equivariant Dirac operators on noncompact manifolds. K-Theory, 27 (2002), 61-101.

[7] - The index theory on non-compact manifolds with proper group action. J. Geom. Phys., 98 (2015), 275-284.

[8] Duflo, M., Construction de représentations unitaires d'un groupe de Lie, in Harmonic Analysis and Group Representations, pp. 129-221. Liguori, Naples, 1982.

[9] Duistermate, J. J., The Heat Kernel Lefschetz Fixed Point Formula for the Spin-c Dirac Operator. Progress in Nonlinear Differential Equations and their Applications, 18. Birkhäuser, Boston, MA, 1996.

[10] Grossberg, M. D. \& Karshon, Y., Bott towers, complete integrability, and the extended character of representations. Duke Math. J., 76 (1994), 23-58.

[11] - Equivariant index and the moment map for completely integrable torus actions. Adv. Math., 133 (1998), 185-223.

[12] Gulllemin, V., Reduced phase spaces and Riemann-Roch, in Lie Theory and Geometry, Progr. Math., 123, pp. 305-334. Birkhäuser, Boston, MA, 1994.

[13] Gulllemin, V. \& Sternberg, S., Geometric quantization and multiplicities of group representations. Invent. Math., 67 (1982), 515-538.

[14] - A normal form for the moment map, in Differential Geometric Methods in Mathematical Physics (Jerusalem, 1982), Math. Phys. Stud., 6, pp. 161-175. Reidel, Dordrecht, 1984.

[15] Hattori, A., Spin ${ }^{c}$-structures and $S^{1}$-actions. Invent. Math., 48 (1978), 7-31. 
[16] Hochs, P. \& Mathai, V., Quantising proper actions on $\operatorname{Spin}^{c}$-manifolds. Asian J. Math., 21 (2017), 631-686.

[17] Hochs, P. \& Song, Y., Equivariant indices of Spin ${ }^{c}$-Dirac operators for proper moment maps. Duke Math. J., 166 (2017), 1125-1178.

[18] Jeffrey, L. C. \& Kirwan, F. C., Localization and the quantization conjecture. Topology, 36 (1997), 647-693.

[19] Karshon, Y. \& Tolman, S., The moment map and line bundles over presymplectic toric manifolds. J. Differential Geom., 38 (1993), 465-484.

[20] Kirwan, F. C., Cohomology of Quotients in Symplectic and Algebraic Geometry. Mathematical Notes, 31. Princeton University Press, Princeton, NJ, 1984.

[21] Lerman, E., Meinrenken, E., Tolman, S. \& Woodward, C., Nonabelian convexity by symplectic cuts. Topology, 37 (1998), 245-259.

[22] Ma, X. \& Zhang, W., Geometric quantization for proper moment maps: the Vergne conjecture. Acta Math., 212 (2014), 11-57.

[23] Meinrenken, E., Symplectic surgery and the Spin ${ }^{c}$-Dirac operator. Adv. Math., 134 (1998), 240-277.

[24] Meinrenken, E. \& SjamaAr, R., Singular reduction and quantization. Topology, 38 (1999), 699-762.

[25] Paradan, P.-E., Formules de localisation en cohomologie equivariante. Compositio Math., 117 (1999), 243-293.

[26] - Localization of the Riemann-Roch character. J. Funct. Anal., 187 (2001), 442-509.

[27] Paradan, P.-E. \& Vergne, M., Index of transversally elliptic operators. Astérisque, 328 (2009), 297-338.

[28] - Witten non abelian localization for equivariant $K$-theory and the $[Q, R]=0$ theorem. To appear in Mem. Amer. Math. Soc.

[29] - Admissible coadjoint orbits for compact lie groups. To appear in Transform. Groups.

[30] Cannas da Silva, A., Karshon, Y. \& Tolman, S., Quantization of presymplectic manifolds and circle actions. Trans. Amer. Math. Soc., 352 (2000), 525-552.

[31] SjamaAR, R., Holomorphic slices, symplectic reduction and multiplicities of representations. Ann. of Math., 141 (1995), 87-129.

[32] Teleman, C., The quantization conjecture revisited. Ann. of Math., 152 (2000), 1-43.

[33] Tian, Y. \& Zhang, W., An analytic proof of the geometric quantization conjecture of Guillemin-Sternberg. Invent. Math., 132 (1998), 229-259.

[34] Vergne, M., Multiplicities formula for geometric quantization. I, II. Duke Math. J., 82 (1996), 143-179, 181-194.

[35] — Equivariant index formulas for orbifolds. Duke Math. J., 82 (1996), 637-652.

[36] Witten, E., Two-dimensional gauge theories revisited. J. Geom. Phys., 9 (1992), 303-368.

\section{Paul-Emile Paradan}

Institut Montpelliérain A. Grothendieck CNRS UMR 5149

Université de Montpellier

FR-34095, Montpellier

France

paul-emile.paradan@umontpellier.fr

Received December 14, 2015
MichèLe VERGNe

Institut de Mathématiques de Jussieu

CNRS UMR 7586

Université Paris 7

FR-75205, Paris

France

vergne@math.jussieu.fr 